\title{
X-ray spectroscopy of galaxy clusters: studying astrophysical processes in the largest celestial laboratories
}

\author{
Hans Böhringer • Norbert Werner
}

Received: 8 April 2009 / Accepted: 23 June 2009 / Published online: 2 October 2009 (C) The Author(s) 2009. This article is published with open access at Springerlink.com

\begin{abstract}
Galaxy clusters, the largest clearly defined objects in our Universe, are ideal laboratories to study in detail the cosmic evolution of the intergalactic intracluster medium (ICM) and the cluster galaxy population. For the ICM, which is heated to X-ray radiating temperatures, X-ray spectroscopy is the most important tool to obtain insight into the structure and astrophysics of galaxy clusters. The ICM is also the hottest plasma that can be well studied under thermal equilibrium conditions. In this review we recall the basic principles of the interpretation of X-ray spectra from a hot, tenuous plasma and we illustrate the wide range of scientific applications of $\mathrm{X}$-ray spectroscopy. The determination of galaxy cluster masses, the most important prerequisite for using clusters in cosmological studies, rest crucially on a precise spectroscopic determination of the ICM temperature distribution. The study of the thermal structure of the ICM provides a very interesting fossil record of the energy release during galaxy formation and evolution, giving important constraints on galaxy formation models. The temperature and pressure distribution of the ICM gives us important insight into the process of galaxy cluster merging and the dissipation of the merger energy in form of turbulent motion. Cooling cores in the centers of about half of the cluster population are interesting laboratories to investigate the interplay between gas cooling, star- and black hole formation and energy feedback, which is diagnosed by means of X-ray spectroscopy. The element abundances deduced from X-ray spectra of the ICM provide a cosmic history record of the contribution of different supernovae to the nucleosynthesis of heavy elements and their spatial distribution partly reflects
\end{abstract}

\footnotetext{
H. Böhringer $(\varangle)$

Max-Planck-Institut für extraterrestrische Physik, 85748 Garching, Germany

e-mail: hxb@mpe.mpg.de
}

N. Werner

Kavli Institute for Particle Astrophysics and Cosmology,

Stanford University, 452 Lomita Mall, Stanford, CA 94305, USA 
important transport processes in the ICM. Some discussion of plasma diagnostics for conditions out of thermal equilibrium and an outlook on the future prospects of X-ray spectroscopic cluster studies complete our review.

Keywords X-ray astronomy - Galaxies: clusters of galaxies $\cdot$ Spectroscopy: X-rays

\section{Introduction}

Galaxy clusters are contrary to their name more than just a collection of galaxies. In the 1930s, Zwicky (1937) discovered that it requires a large amount of unseen matter to bind the fast moving galaxies in the Coma galaxy cluster into a long lasting object. Today we have a clear cosmic scenario with galaxy clusters as an integral part of the large-scale structure of the Universe. They are the largest matter aggregates within the large-scale structure which have collapsed under their own gravity and are closely approaching a dynamical equilibrium. Much theoretical effort has been spent to understand and characterize this equilibrium structure. To the first order, galaxy clusters are now described as Dark Matter Halos with a characteristic universal shape of the Dark Matter potential (e.g. Navarro et al. 1995; Moore et al. 1999; Gao et al. 2008). Observations show indeed, that the cluster population can be described as constituting a nearly self-similar family with similar shapes of the matter distribution, where the small scatter is due to the different formation histories and differently close approaches to the equilibrium configuration.

Galaxy clusters are therefore very important giant astrophysical laboratories providing us with a well characterized physical environment in which we can study many interesting astrophysical phenomena and cosmic processes on giant scales (Sarazin 1986). They also allow us to study large coeval galaxy populations and enable us to investigate their evolution in connection with the chemical and thermal evolution of the embedding intracluster medium (ICM) (e.g. Dressler 1980; Dressler et al. 1997; Poggianti et al. 1999; Mei et al. 2006).

As tracers of the cosmic large-scale structure they are also important probes for cosmology. It is the growth of structure in the matter distribution of the Universe that has a strong dependence on the cosmological model parameters and in particular on the nature of Dark Matter and Dark Energy. Since galaxy clusters are very sensitive tracers of structure growth, a census of the cluster population as a function of redshift can be used to test cosmological models (e.g. Borgani et al. 2001; Schuecker et al. 2003a,b; Henry 2004; Vikhlinin et al. 2003, 2009; Henry et al. 2009).

The current most important limitation in using galaxy cluster studies for cosmology is the calibration of the relation between various observables and cluster mass. Therefore a lot of effort is currently being spent to improve the cluster mass determination and the understanding of cluster structure (e.g. Arnaud et al. 2007; Vikhlinin et al. 2006; Pratt et al. 2006, 2007). X-ray spectroscopy of the cluster emission plays a crucial technical role in this effort to characterize cluster structure precisely, to model the cluster population as a family of self-similar objects with explainable deviations, and to establish scaling relations of global cluster parameters that allow to draw comprehensive statistical conclusions on cluster properties from simple observables. 


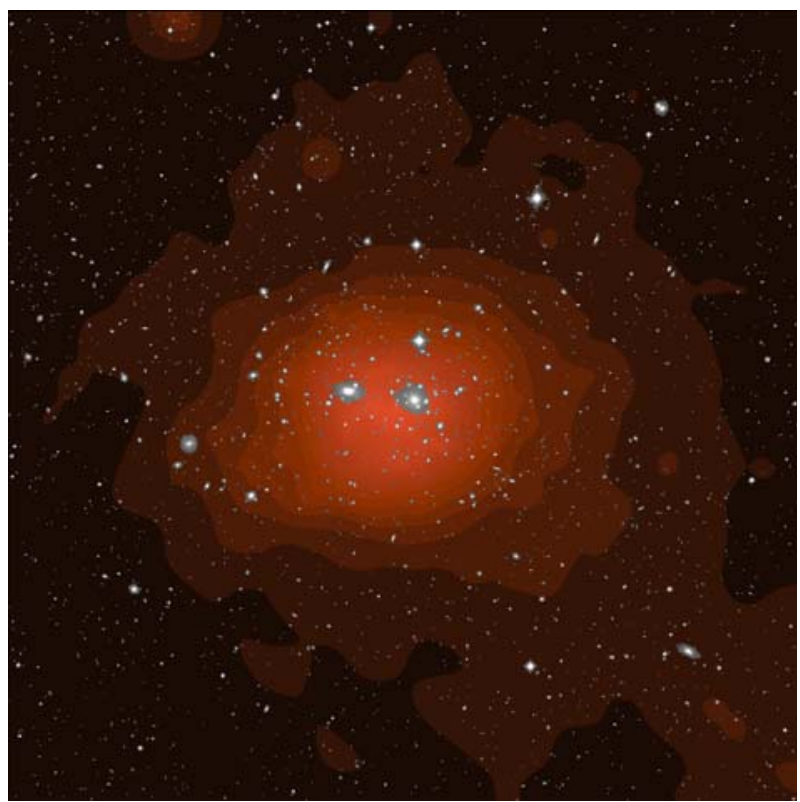

Fig. 1 The Coma cluster of galaxies as seen in X-rays in the ROSAT All-Sky Survey (underlaying red color) and the optically visible galaxy distribution in the Palomar Sky Survey Image (galaxy and stellar images from the digitized POSS plate superposed in grey)

Most of the detailed knowledge on galaxy clusters has been obtained in recent years through X-ray astronomy. This is due to the fact that the ICM has been heated to temperatures of tens of Millions of degrees (several keV per particle) which causes the hot plasma to emit the bulk of the thermal energy in the regime of soft X-rays. Since this is also the photon energy range where the well developed X-ray telescopes come into play, galaxy clusters are among the most rewarding study objects for X-ray imaging and spectro-imaging observations. Figure 1 shows a composite image of the Coma galaxy cluster, where an optical image from the Palomar Sky Survey showing the dense galaxy distribution of the Coma cluster is superposed in grey scale on top of an X-ray image from the ROSAT All-Sky Survey with X-ray brightness coded in red color. We clearly recognize that the X-ray image displays the cluster as one connected entity. This illustrates the fact that galaxy clusters are well defined, fundamental building blocks of our Universe.

As largest cosmic objects they display several interesting astrophysical superlatives: (i) the ICM is the hottest thermal equilibrium plasma that we can study in detail, with temperatures up to two orders of magnitude larger than the temperature in the center of the sun, (ii) the gravitational potential of clusters gives rise to the largest effect of light deflection with deflection angles exceeding half an arcmin, producing the most spectacular gravitational lensing effects (e.g. Hattori et al. 1999), (iii) the hot plasma cloud of the ICM casts the darkest shadows onto the cosmic microwave background through the Sunyaev-Zeldovich effect (at wavelengths below about $1.4 \mathrm{~mm}$ they are seen as surface brightness enhancements) (e.g. Birkinshaw 1999), and (iv) the merger 
of galaxy clusters produces the largest energy release in the Universe after the big bang itself with energies up to orders of $10^{63} \mathrm{erg}$ (e.g. Feretti et al. 2002).

$\mathrm{X}$-ray observations and X-ray spectroscopy are the most important tools to obtain detailed information on cluster properties and the processes occurring in their ICM as will be illustrated in this article. In Sect. 2 we will explain how observable X-ray spectra can be understood and modeled. In Sect. 3 we show how this spectral modeling is applied to study the thermal structure of the ICM that also provides the tool for measuring cluster masses. Section 4 deals with the diagnostics of the central regions of clusters where massive cooling is prevented by AGN heating in those objects where the cooling time is short enough for effective cooling. In Sect. 5 we illustrate how the observed spectral lines can be used for the chemical analysis of the ICM and what can be learned from these observations. In Sect. 6 we take a look into the physics of plasma under non-equilibrium ionization conditions and in the last section we provide an outlook on the capabilities and potential of X-ray instruments planned for the future.

\section{X-ray spectra of hot tenuous plasma}

The plasma of the ICM is very tenuous, with densities of $10^{-5}-10^{-1} \mathrm{~cm}^{-3}$ from the cluster outskirts to the densest regions of cool core (CC) clusters. This low plasma density makes the cluster X-ray spectra modeling simple and enables a very straightforward interpretation. Three fundamental emission processes involving electronic "transitions" contribute to the radiation: free-free or bremsstrahlung radiation caused by the deflection of an electron at close fly-by of an ion, free-bound or recombination radiation caused by the capture of an electron by an ion following ionization, and bound-bound or deexcitation radiation of an electron changing the quantum level in an ion. The first two processes give rise to continuum radiation and the latter to line radiation. An exception in bound-bound transitions is the radiative transition from the $2 \mathrm{~s}$ to the $1 \mathrm{~s}$ state, which is completely forbidden by angular momentum conservation, but can happen as very slow two-photon process. This "two-photon radiation" involves a distribution function of the branching ratio of the energies of the two electrons (Spitzer and Greenstein 1951) and thus gives rise to continuum radiation.

We note that all these radiative processes depend on the collision (or close fly-by) of an electron and an ion. Due to the very low density of the plasma all the ions excited by collisions have sufficient time for radiative deexcitation before a second deexciting collision occurs. Thus contrary to laboratory plasmas, where slow transitions are "forbidden" and the corresponding excited states are much more rapidly deexcited by electronic collision, all "forbidden" transitions actually happen in the ICM plasma. This leads to a scenario where all exciting, recombining, and bremsstrahlung causing collisions lead to the radiation of a photon, which is referred to as the thin plasma radiation limit (or "coronal limit", as similar conditions prevail in the solar corona). The modeling of the thermal plasma spectrum is therefore a book keeping exercise of all the electron ion collisions rates and-in the case of several deexcitation channelstheir branching ratios. These collision rates are in general a function of temperature (for thermal plasma) and the outcome is directly proportional to the electron density 
and the respective ion density. The shape of the resulting spectrum is therefore a function of the temperature and chemical composition and its normalization is directly proportional to the electron density and the ion density. The latter is true for the low plasma density limit that pertains in the ICM.

We further note that in general all photons so created leave the ICM plasma due to its low density. An exception is discussed in Sect. 5.5.3 for the strongest lines in dense cluster cores. Thus no radiative transfer calculation is necessary for the interpretation of the X-ray spectra of the ICM. This means in particular that the spectrum we observe from a galaxy cluster provides an account of the entire ICM plasma, which is very different from, e.g. the spectra of stars, that provide information on merely a very thin skin on their surface. This is another reason why cluster X-ray spectra are so informative and straightforward to interpret.

As the radiation contribution specific to an ion species is proportional to the density of this species in the plasma, the modeling of the overall spectrum also requires the knowledge of the chemical composition of the plasma and its ionization structure. For thermal equilibrium plasma, the ionization structure is characterized by the balance of the ionization and recombination rates for all the ionization steps of an element:

$$
\begin{aligned}
& {\left[I^{n+}\right] n_{e}\left(C_{c i}+C_{a i}\right)+\left[I^{n+}\right]\left(\left[H^{+}\right] X_{c e 1}+\left[H e^{+}, H e^{++}\right] X_{c e 2}\right)} \\
& \quad=\left[I^{(n+1)+}\right] n_{e}\left(C_{r r}+C_{d r}\right)+\left[I^{(n+1)+}\right]\left([H] X_{c e 3}+[H e] X_{c e 4}\right) .
\end{aligned}
$$

Several processes contribute to the ionization and recombination rate. The ionization comprises direct electron impact ionization, $C_{c i}$, electron impact excitation into an auto-ionization state with subsequent auto-ionization, $C_{a i}$, and ionizing charge exchange with $\mathrm{H}^{+}, \mathrm{He}^{+}$, and $\mathrm{He}^{++}$with the rates $\mathrm{X}_{c e 1}$ and $\mathrm{X}_{c e 2}$, respectively. Direct ionization is the dominant process. Recombination rates include radiative recombination, $C_{r r}$, dielectronic recombination, $C_{d r}$, and electron capture charge exchange with $H$ and $H e$, with the rates $X_{c e}$ and $X_{c e}$. In the dielectronic recombination process the recombination collision complex is energetically stabilized by the excitation of a second bound electron which subsequently radiates the excess energy. This is a resonant process which exhibits a strong energy dependence which is most important for ions with many electrons (e.g. Fe ions). The ionization structure is calculated from the complete set of linear equations for all important ions and elements (e.g. Arnaud and Rothenflug 1985; Arnaud et al. 1992; Mazzotta et al. 1998). Figure 2 shows for example the thermal equilibrium ionization structure of oxygen in the temperature range $10^{4}-10^{8} \mathrm{~K}$ (Böhringer 1998).

The collision rates that lead to the emission of radiation are then all of the form:

$$
R=n_{e} n_{I} C_{I x}=n_{e}^{2}\left[\frac{n_{I}}{n_{E}}\right]\left[\frac{n_{E}}{n_{H}}\right]\left[\frac{n_{H}}{n_{e}}\right] C_{I x}(T),
$$

where $n_{E}, n_{I}$, and $n_{H}$ are the number densities of the elements, ions, and hydrogen nuclei, respectively, and $C_{I x}$ are the relevant collision rate coefficients for ion $I$. Consequently, $\left[\frac{n_{I}}{n_{E}}\right]$ represents the fractional abundance of ionization stage $I,\left[\frac{n_{E}}{n_{H}}\right]$ the 


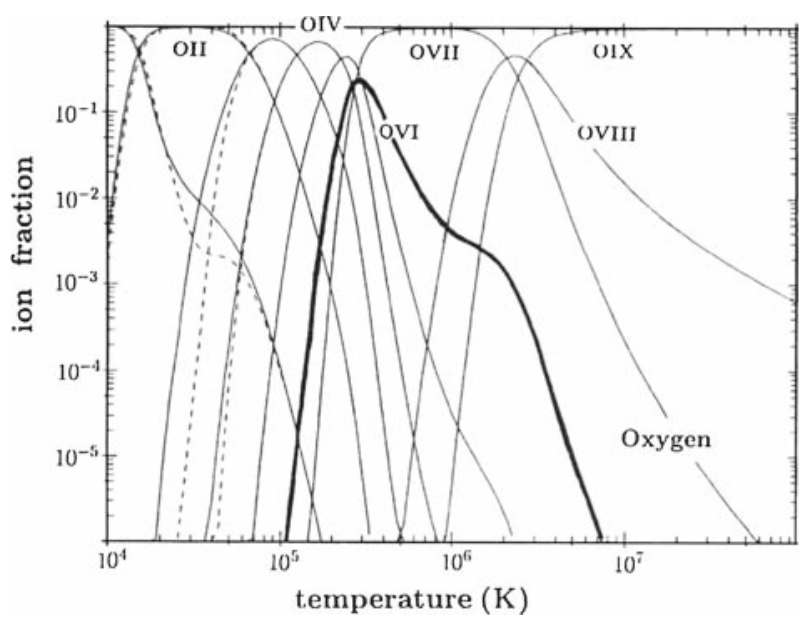

Fig. 2 Thermal equilibrium ionization structure of the oxygen ion family as a function of plasma temperature (Böhringer 1998)

relative elemental abundance, and $\left[\frac{n_{H}}{n_{e}}\right]$ the Hydrogen nuclei to electron ratio, which is about 1.2. Thus all rates are proportional to the squared plasma density, $n_{e}^{2}$. The normalization of the spectrum is therefore determined from the emission measure, $E$, given by:

$$
E=\int n_{e}^{2} \mathrm{~d} V
$$

We note that this definition of emission measure is not unique. It conforms to most definitions in text books, but the major public plasma radiation codes use the definition $E=\int n_{e} n_{H} \mathrm{~d} V$. The strategy of calculating the radiation spectrum of hot, thin plasma in thermal equilibrium is thus summarized in Fig. 3. The notion of thermal equilibrium involves a thermal Maxwellian equilibrium of the electrons and the thermal ionization equilibrium of the ions. A deviation of the temperature of the electrons and the ions will not affect the components of the radiation spectrum, but will be detected through thermal line broadening.

With a change of the temperature of the plasma the ionization structure is adjusted on the time scale of the inverse ionization and recombination rates and thus lags behind the temperature change. In certain cases this time lag is significant and has to be included in the modeling, as sketched in Fig. 3 by explicitly following the time evolution of the ionization structure. As an example, Fig. 4 shows the calculation of a passively cooling plasma starting at high temperature (Shapiro and Moore 1976) where the instantaneous ionization equilibrium assumption is compared to the full time dependent ionization structure calculations. We see that strong deviations occur at temperatures of the order of $10^{5} \mathrm{~K}$, but no significant deviation from the ionization equilibrium calculations are observed above $10^{6} \mathrm{~K}$, which is the temperature range in clusters. So far no observed X-ray spectrum of the ICM has required a non-ionization 


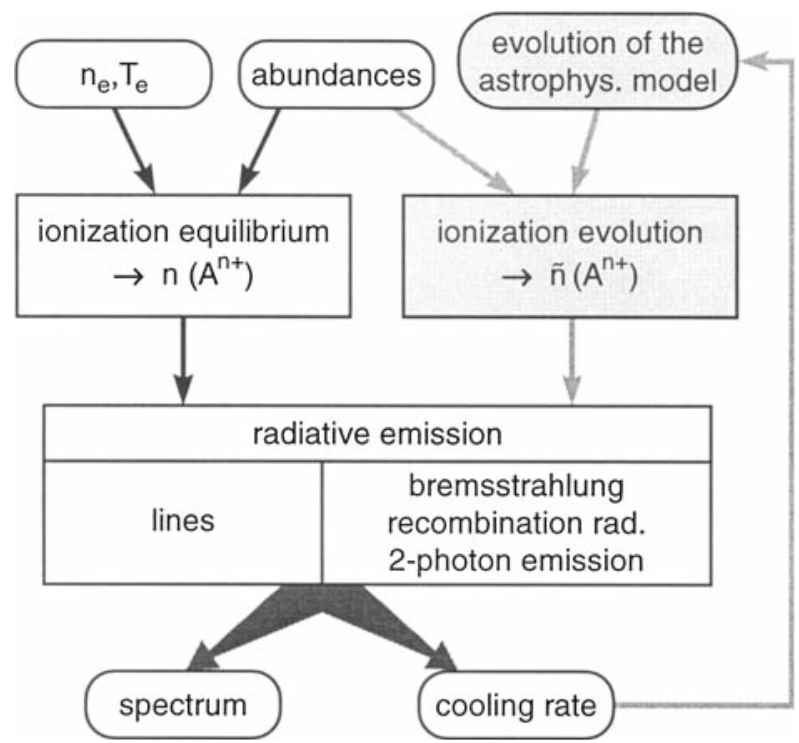

Fig. 3 Scheme of the calculation of the radiation spectrum and cooling function of a hot, tenuous plasma in thermal equilibrium. The shaded boxes include the situation of non-equilibrium ionization (from Böhringer 1998)

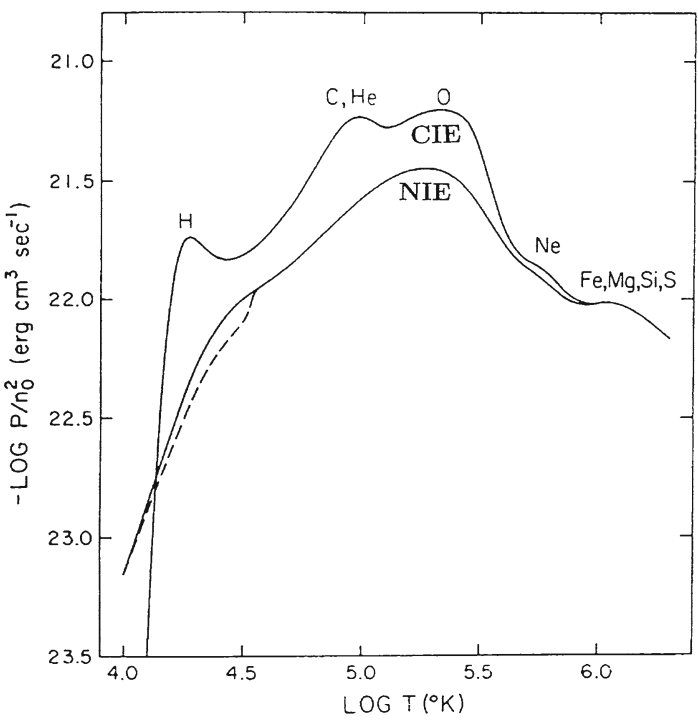

Fig. 4 Cooling coefficient of hot plasma as a function of the electron temperature from (Shapiro and Moore 1976). Two scenarios of the cooling of plasma from an initially hot phase of $T \sim 10^{7} \mathrm{~K}$ are compared. In the CIE (collisional ionization equilibrium) case the ionization structure is kept in full thermal equilibrium, in the NIE (non-ionization equilibrium) case on the contrary, the ionization structure passively follows the cooling plasma and recombination can lag behind the cooling. The latter implies higher ionization stages at the same temperature, that is less shell electrons and reduced line cooling 


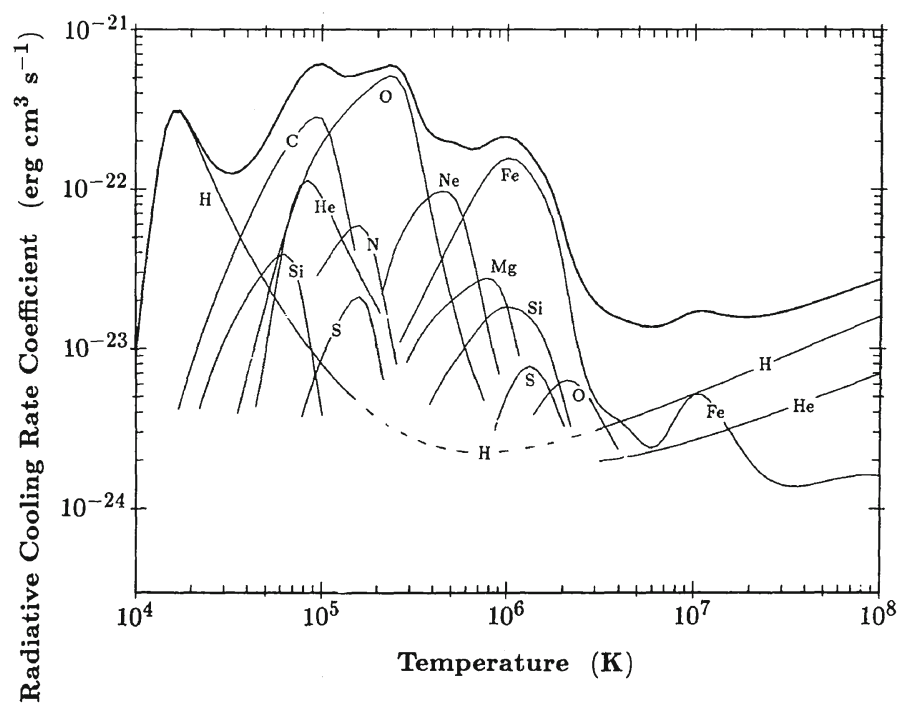

Fig. 5 Cooling rate of hot plasma as a function of the plasma temperature. The contribution to the cooling by the ions of different important abundant elements is indicated (Böhringer and Hensler 1989). Most of this contribution is in the form of line radiation, which is by far the dominant form of radiation in the temperature range from about $10^{4}$ to $2 \times 10^{6} \mathrm{~K}$. At higher temperatures over most of the regime of interest for the ICM the Bremsstrahlung contribution dominates

equilibrium treatment. Figure 5 shows the contributions of continuum and line emission and the respective contributions of the most important elements (assuming solar abundances) to the total radiation power. The regime in which the recombination lags behind cooling can easily be identified with the temperature range where line radiation from the important, most abundant elements (particularly Fe) is strongly boosting the line cooling. We note that continuum bremsstrahlung with a temperature dependence of roughly $\propto T^{1 / 2}$ is the dominant radiation process at ICM temperatures.

Early modeling of the emission spectrum of hot, thin plasma include: Cox and Tucker (1969), Tucker and Koren (1971), Cox and Daltabuit (1971), Cox (1972), Shapiro and Moore (1976, 1977), Kato (1976), Raymond and Smith (1977), Landini and Monsignori Fossi (1970, 1990), Landini and Fossi (1991), Gaetz and Salpeter (1983), Raymond (1988), Masai (1984), Masai and Kato (1987), Böhringer and Hensler (1989), Schmutzler and Tscharnuter (1993), Sutherland and Dopita (1993), Brickhouse et al. (1995), and Raymond and Brickhouse (1996). Now the two most frequently used plasma radiation codes are the MEKAL code (Gronenschild and Mewe 1978; Mewe and Gronenschild 1981; Mewe et al. 1985; Mewe 1990, 1991; Kaastra and Mewe 1994; Mewe et al. 1995) and the APEC code (Smith et al. 2001). While the older literature contains all the necessary information about the radiation codes including all the details of the rate calculations and much detail on the underlying physical processes, the modern codes are too complex to be easily described in the literature. Thus, unfortunately very little documentation about the new development of the radiation codes is available in written form after the mid 1990s.

Figure 6 shows typical spectra at temperatures of $10^{5}, 10^{6}, 10^{7}$, and $10^{8} \mathrm{~K}$ with solar element abundances, indicating the contribution by line radiation and the continuum 

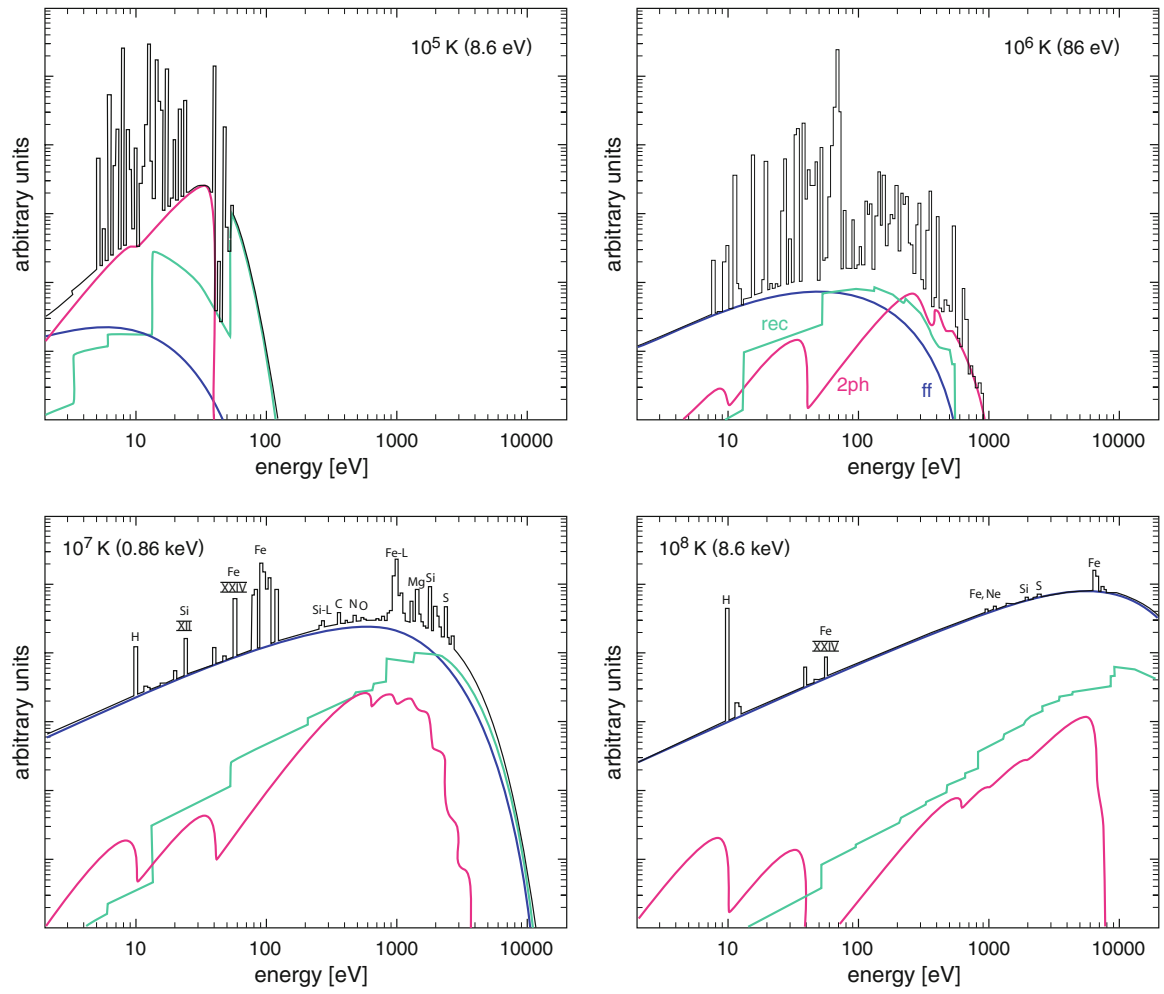

Fig. 6 X-ray spectra for solar abundance at different plasma temperatures. The continuum contributions from bremsstrahlung (blue), recombination radiation, characterized by the sharp ionization edges (green), and 2-photon radiation (red) are indicated. At the highest temperatures relevant for massive clusters of galaxies bremsstrahlung is the dominant radiation process (from the work described in Böhringer and Hensler 1989). The major emission lines in the panels for the higher temperatures relevant for galaxy clusters are designated by the elements from which they originate (The labels Fe-L ans Si-L refer to transitions into the $\mathrm{L}$-shell in ions of $\mathrm{Fe}$ and $\mathrm{Si}$, respectively, and two other lines with roman numbers carry the designation of the ions from which they originate involving transitions within the L-shells

emission from bremsstrahlung, recombination and two-photon transitions. We clearly see the increasing dominance of bremsstrahlung with increasing temperature, which reflects the fact that fewer ions retain electrons and the plasma is almost completely ionized at the higher temperatures.

\section{The study of the thermal structure of the intracluster medium}

We have seen in the previous section that the shape of the spectrum for a thermal equilibrium plasma is determined by the plasma temperature and the elemental abundances. This is therefore the basic information we derive from the spectral analysis of the ICM radiation: a temperature measurement and a chemical analysis. We consequently illustrate in this, and the next chapter, the scientific insights gained from temperature measurements from the state-of-the-art spectral analysis, and in Sect. 5 the lessons learned from the chemical analysis of the ICM. 


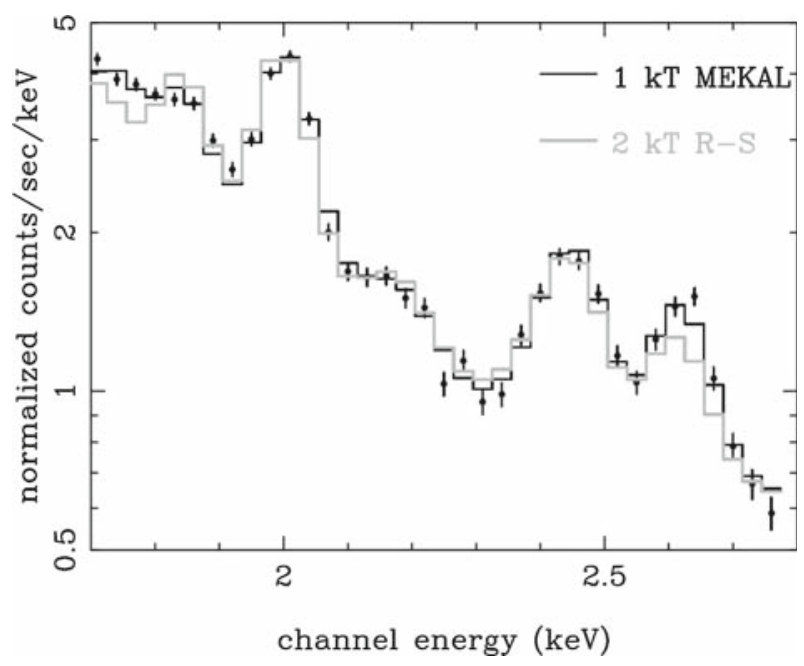

Fig. 7 X-ray spectrum of the ICM plasma in the halo of M87 at a temperature around $1.7 \mathrm{keV}$ observed with XMM-Newton EMOS, showing exclusively the spectral range with the $\mathrm{H}$ - and He-like lines of Si and $\mathrm{S}$ (Matsushita et al. 2002). The spectrum is fitted with a one-temperature MEKAL model (black line) and a two-temperature model with the temperature components 1.4 and $3.3 \mathrm{keV}$ (grey line). The one-temperature model provides a better fit, supporting the view that the temperature distribution is very narrow. For more details see the original publication

It is mostly the shape of the continuum spectrum, dominated by bremsstrahlung, that provides information on the temperature. The spectral energy distribution for the thermal bremsstrahlung spectrum for the collision of an electron with ion, $i$, is given by (e.g. Gronenschild and Mewe 1978):

$$
\epsilon(\nu)=\frac{16 e^{6}}{3 m_{e} c^{2}}\left(\frac{2 \pi}{3 m_{e} k_{B} T_{X}}\right)^{1 / 2} n_{e} n_{i} Z^{2} g_{f f}\left(Z, T_{X}, v\right) \exp \left(\frac{-h v}{k_{B} T_{X}}\right)
$$

where $m_{e}$ and $n_{e}$ are the electron mass and density, respectively, $n_{i}$ is the respective ion density, $Z$ is the effective charge of the ion, and $g_{f f}$ is the gaunt factor, a quantity close to unity which must be calculated numerically through a quantum-mechanical treatment. The most prominent spectral signature of $T_{X}$ is the sharp cut-off of the spectrum at high energies, due to the exponential term with the argument $-h v / k_{B} T$. As long as this cut-off is seen in the energy window of the telescope, one has a good handle on the temperature measurement. The element abundances are mostly reflected in the intensity of the spectral lines. Only in well resolved spectra of very high quality, where we can observe several lines of the same element, is the temperature also constrained by line strength ratios. A prominent such case occurs at temperatures around 2-3 keV where we can observe the K-shell and L-shell lines of iron simultaneously in high photon count spectra. Another example of temperature diagnostics based on the study of the hydrogen and helium like Si and S lines is shown in Fig. 7 (taken from Matsushita et al. 2002). This work provides a further nice illustrations of how different temperature indicators can be used to check the consistency of the temperature measurement and to test if the plasma has a distribution of temperature phases. 
The first X-ray spectral observations of clusters lacked sufficient angular resolution and thus involved the total ICM emission of the targets and only provided us with information on the bulk temperature of the cluster ICM. What does this parameter tell us about a cluster? Galaxy clusters form from the gravitational collapse of overdense regions in the matter density distribution in the Universe and subsequently approach an equilibrium configuration characterized by a virial relation:

$$
E_{\text {kinetic }}=-2 E_{\text {potential }} \propto \frac{G M}{R}
$$

where the mass, $M$, refers to the total mass of the galaxy clusters including the dark matter. Analogously to the virial equilibrium of galaxies and dark matter particles, the ICM plasma thermalizes and attains a "virial temperature" which reflects the depth of the gravitational potential of the cluster. In the collapse process the potential energy of the ICM is converted to internal heat. If the gravitational potentials of clusters of different mass have a self-similar shape, as implied by numerical simulations of gravitational collapse (e.g. Navarro et al. 1995; Moore et al. 1999), then one finds the following self-similar relation between cluster mass and ICM temperature:

$$
T \propto \sigma_{D M}^{2} \propto M / R \propto M^{2 / 3}
$$

where $\sigma_{D M}$ is the velocity dispersion of the dark matter particles. (The rightmost relation is due to $M \propto R^{3}$ and $\rho_{D M}=$ const. in the self-similar model.)

The analysis of the first galaxy cluster spectra indirectly confirmed this trend by showing that the ICM temperature increases with the galaxy velocity dispersion. Figure 8 shows one of the first such relations (Mushotzky 1984) with X-ray spectral observations from the HEAO-1 A2 satellite experiment. Modern versions of these relations-given here directly as $M-T_{X}$ relations-are shown in Fig. 9 for selected regular clusters from Arnaud et al. (2007) and for cluster sample from Kotov and Vikhlinin (2006), respectively. The spectroscopically determined ICM temperature thus turns out to be one of the best mass proxies as single observable parameter (e.g. Kravtsov et al. 2006). The tightest relations are obtained, if the core regions are excluded in the global temperature measurement, due to the disproportionate influence of the central CCs as will become apparent below, and it has thus become standard to quote the mean temperature in the radial region $r=0.15-1 \times r_{500}$ as the most reliable single observable mass proxy for clusters (e.g. Arnaud et al. 2005; Pratt et al. 2009). ${ }^{1}$

The advanced X-ray observatories Chandra and XMM-Newton now routinely also provide localized measurements of the ICM temperature. First attempts had already been made using observations with the ROSAT observatory, e.g. to produce temperature maps featuring still large temperature uncertainties (Briel and Henry 1994; Henry and Briel 1995) and later by means of the ASCA observatory, but due to its comparably low angular resolution most of the results remained somewhat ambiguous and depended

\footnotetext{
${ }^{1} r_{500}$ is the radius where the mean total density of the cluster is 500 times the mass density of a Universe with critical density. This radius describes the same relative scale of clusters of different mass in the self-similar scenario. See also explanation later in the text.
} 


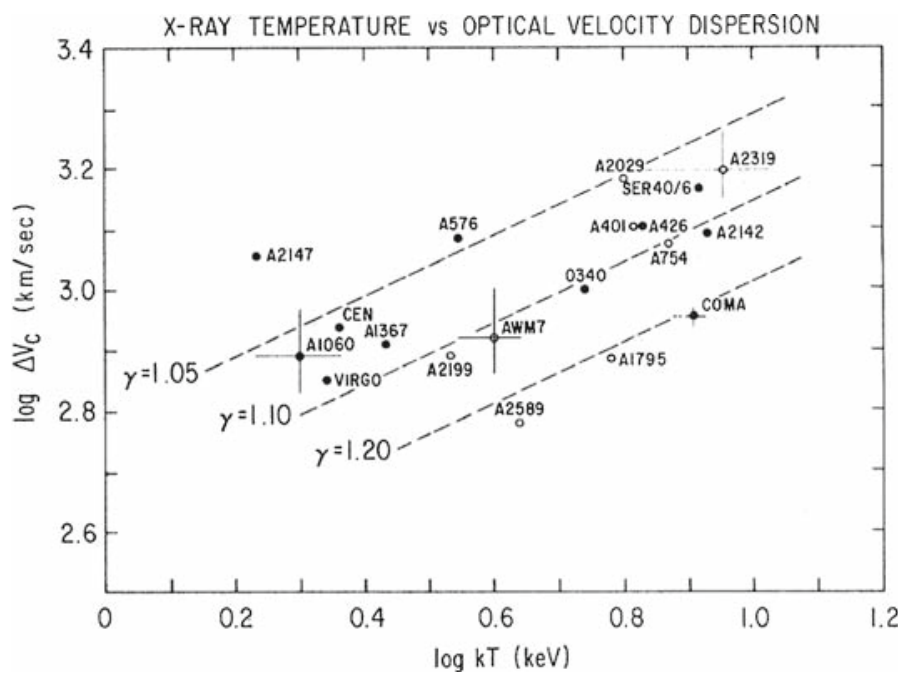

Fig. 8 Correlation of the ICM temperature with the velocity dispersion of the galaxies in clusters (Mushotzky 1984). The temperatures have been derived from X-ray spectra taken with the HEAO-1 satellite A-2 experiment. The dots are data for non-cD clusters and the open symbols for $\mathrm{cD}$ clusters (clusters with a central dominant, $\mathrm{cD}$ type galaxy). The lines show predictions of polytropic models with various indices (see Mushotzky 1984)
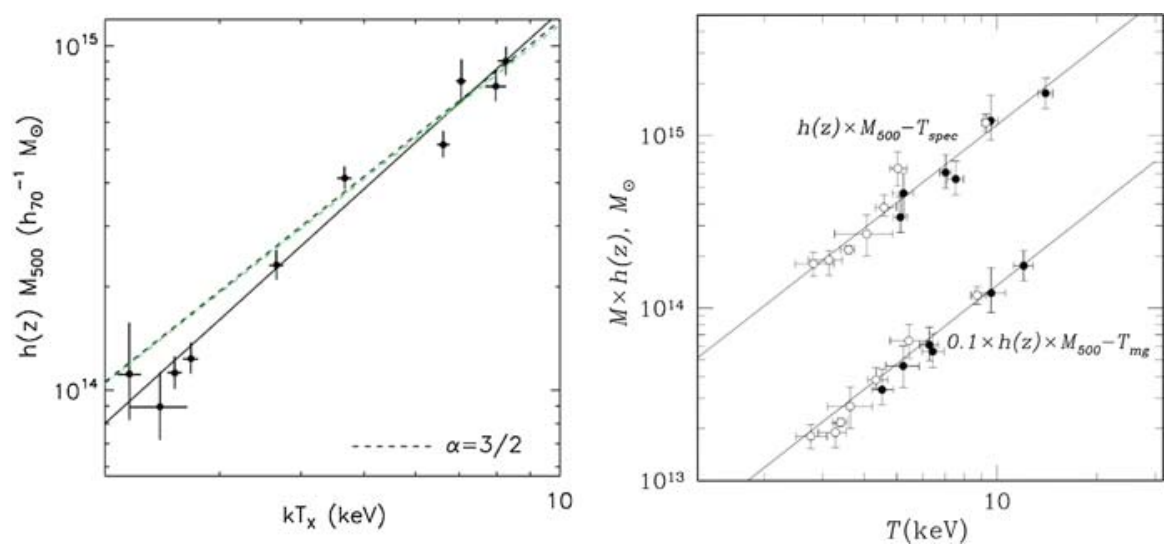

Fig. 9 Left The mass-temperature relation of a sample of regular clusters from (Arnaud et al. 2007). The best fitting slope (solid line) is $1.71 \pm 0.09$ slightly steeper than the self-similar relation. Right The masstemperature relation of galaxy clusters and its evolution with redshift derived from two cluster samples at $\langle z\rangle \sim 0.05$ and $\langle z\rangle \sim 0.55$ (Kotov and Vikhlinin 2006). The relations have been displaced by a factor of 10 for better visibility. The model predicted redshift evolution (clusters of equal mass get hotter with look-back time) has been accounted for by scaling with the factor $h(z)$. This predicted evolutionary trend is well supported by the data

on the analysis method used (e.g. Ikebe et al. 1997; Markevitch et al. 1998; White 2000). With some studies by means of the Italian-Dutch X-ray mission BeppoSAX some overview on the temperature structure of clusters was obtained, with decreasing temperature profiles outside $\sim 0.2 r_{500}$ and a diversity of the behavior in the core 

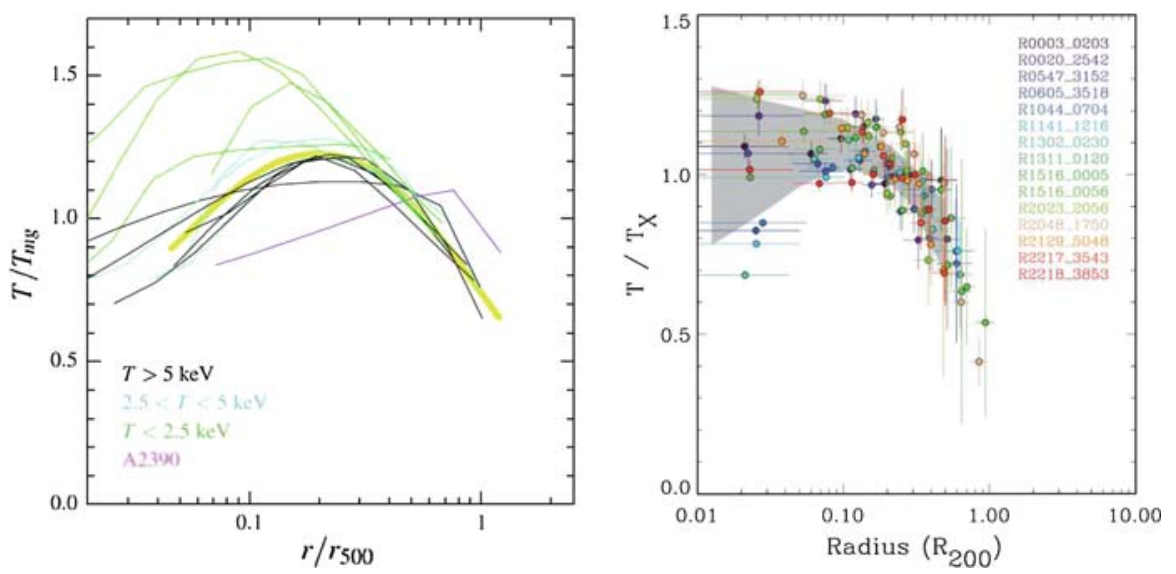

Fig. 10 Scaled temperature profiles of galaxy clusters derived from spectroscopic observations with the Chandra satellite (left, Vikhlinin et al. 2006) and results obtained for a sample of clusters observed with XMM-Newton (right, Pratt et al. 2007)

region depending on the thermal history of the cluster (De Grandi and Molendi 2002). This picture has become very precise with systematic studies with XMM-Newton and Chandra. Figure 10 shows results on the temperature profiles of cluster samples studied with both observatories (Vikhlinin et al. 2006; Pratt et al. 2007).

To better understand these results, we have to make a brief excursion to scaling relations. For the comparison of the structure of galaxy clusters of different mass based on the model of self-similarity mentioned above, we need a fiducial radius which identifies corresponding scales in clusters of different mass. A useful definition of such a scale is the radius at which the mean mass density of the cluster is larger by a certain factor than the critical density of the Universe at the redshift of the cluster. One of the justifications of this picture comes from the spherical collapse model in a critical density universe. A good practical choice for the overdensity factor is 500 (see, e.g. Evrard et al. 1996 who show that inside a region of $\sim r_{500}$ randomized galaxy and mass particle orbits dominate clearly over infalling material), not least from an $\mathrm{X}$-ray observers point of view, since now a larger number of high quality Chandra and XMM-Newton data cover the cluster ICM spectroscopically out to this radius (e.g. Vikhlinin et al. 2006; Pratt et al. 2007). We will most often make use of this scaling radius of $r_{500}$, but also use other values of overdensity depending on the literature from which we draw the examples.

In Fig. 10 the temperature profiles shown are scaled by the overdensity radii (with different overdensity values, which has no importance here). We note that there is a large diversity of the profiles at small radii. There are two classes of galaxy clusters: the clusters with dense ICM cores show temperature profiles with $T_{X}$ decreasing toward the center in the core regions, while in clusters with moderate central densities (typically below $10^{-2} \mathrm{~cm}^{-3}$ ) the temperature profiles are found to be flat or even slightly increasing toward the cluster center. The clusters will be called CC and NCC clusters in the following. Due to the logarithmic display of the radial range, the inner regions 
appear more prominent in Fig. 10. Most of the cluster size and volume is in the outer region, where the temperature profiles become very similar and appear squeezed into a narrow zone in scaled radius. This shows that on large scale the thermal structure of clusters can be viewed as following closely a self-similar model. Characterizing this model and understanding the scatter around the mean model is one of the current important observational goals of X-ray cluster research.

One of the difficulties in deriving the temperature distribution of the ICM in clusters from X-ray spectroscopic observations is the fact that the observed radiation is the result of an integral of radiative emission along the entire line-of-sight through the cluster. Therefore one observational task is to "deproject" the cluster spectra, which can only be done by assuming a certain three-dimensional geometrical shape of the cluster, in general spherical symmetry. The requirements for successful spectral deprojection are very good photon statistics and an angular resolution of the data (instrument point spread function) that is better than the corresponding radial binning used. In general the deprojected spectra are much more noisy than the observed spectra, but most often the deprojection results show only small specific differences to the unprojected data (because most of the emission shaping the spectrum comes from the center-most region).

A further problem in deriving the temperature structure from spectroscopy arises if the plasma has more than one temperature phase locally, that is within the observed patch from which the spectrum is extracted and within the deprojection bin. The question is then: what determines the resulting temperature when this composite spectrum is fit with a single-phase spectral model? A first intuitive guess is to assume that it is an average of the temperatures weighted by the radiative emission contributions of the different phases, the so-called emission weighted temperature, $T_{\text {ew }}$ (e.g. Mathiesen and Evrard 2001). However, as shown by Mazzotta et al. (2004) the resulting fitted temperature is generally biased low compared to $T_{\mathrm{ew}}$. Mazzotta et al. (2004) derive a simple analytical approximation to calculate the fitting result from a temperature mixture with an accuracy of a few percent for ICM temperatures above $3 \mathrm{keV}$. The resulting fitted temperature they call spectroscopic-like temperature, $T_{\mathrm{sl}}$. This approach is based on the fact that the temperature dependence of the spectra according to Eq. 4 can be expressed as:

$$
\epsilon(E, T) \sim n_{e}^{2} \zeta(T, m) T^{-1 / 2} \exp \left(-\frac{E}{k T}\right) \sim n_{e}^{2} \zeta(T, m) T^{-1 / 2}\left(1-\frac{E}{k T}\right)
$$

where $E$ is the photon energy, $m$ is the metallicity (heavy element abundance) of the plasma, and $\zeta(T, m)$ includes the temperature dependence of the gaunt factor and some effect of emission lines. The right hand side is a Taylor series expansion for low enough energies as covered by the energy window of X-ray telescopes (for $k_{B} T>3 \mathrm{keV}$ ). Then it is easy to show that temperature phase addition with subsequent single temperature fitting leads to the following expression for the spectroscopic like temperature:

$$
T_{\mathrm{sl}}=\frac{\int n^{2} T^{\alpha} / T^{1 / 2} \mathrm{~d} V}{\int n^{2} T^{\alpha} / T^{3 / 2} \mathrm{~d} V}
$$


with tests showing that $\alpha=0.75$ is a good approximation. Vikhlinin (2006) has generalized this approach also to low temperatures using a method that requires some more complex numerical simulations that take into account the specifics of the spectroscopic instrument, energy band and galactic absorption providing satisfying results with errors of a few percent.

The latter approach allows us to derive $T_{\mathrm{sl}}$ for a known temperature distribution, but provides no means to correct for a temperature bias, if the distribution is unknown. For example the determination of cluster masses (as explained below) rests on a local average of the pressure which requires a mass weighted temperature average for known density. Since $T_{\mathrm{sl}}$ is always biased low with respect to mass averaging, the mass will be underestimated in the presence of unresolved multi-temperature structure (e.g. Rasia et al. 2005). A study by Rasia et al. (2006) showed that the temperature bias can easily lead to a mass underestimate by $10 \%$ on average. With current instrumentation it is difficult to unveil multi-temperature structure in the temperature range above $3 \mathrm{keV}$. A simple exercise that can be performed with XSPEC is to take an equal emission measure of 4 and $8 \mathrm{keV}$ plasma and to simulate an analysis with the instruments of $X M M-N e w t o n$. Even with a simulated exposure that gives several ten million photons, a single temperature model with $T \sim 5.5 \mathrm{keV}$ gives a perfect fit and we are unable to recover the original input phases. At lower temperatures and very good photon statistics more diagnostics can be performed as seen, e.g. in the study shown in Fig. 7 (Matsushita et al. 2002) and similar studies in other CC regions, e.g. by Fabian et al. (2005) or Simionescu et al. (2009a) shown below, due to the emission lines characteristic to the different temperature phases.

\subsection{Cluster mass determination}

One of the most important applications of the knowledge of the temperature structure in the ICM is the mass determination of galaxy clusters. Assuming the ICM is in hydrostatic equilibrium and the cluster has approximately spherical symmetry, the distribution of the total mass in the cluster is given by the density and temperature profiles by the following equation:

$$
M(r)=-\frac{G k_{B} T_{X}}{\mu m_{p}} r\left(\frac{\log \rho_{g}}{\log r}+\frac{\log T_{X}}{\log r}\right),
$$

where $G$ and $k_{B}$ are the gravitational and Boltzmann's constant, respectively, $\mu$ is the mean particle mass $(\sim 0.6), m_{p}$ the mass of the proton, and $\rho_{g}$ the gas density.

One of the generally interesting results from recent efforts of precise mass determination is the conclusion that the "NFW" dark matter halo model proposed by Navarro et al. $(1995,1997)$ gives a consistent description of the mass distribution of seemingly relaxed clusters (clusters that show a high degree of symmetry in projection and no obvious signs of recent merger activity) as shown in Fig. 11 (Pratt 2006; Vikhlinin et al. 2005, see also Pratt and Arnaud 2002; Pointecouteau et al. 2005; Buote et al. 2007; Voigt and Fabian 2006). First attempts have been made of a rigorous comparison of the cluster masses determined from an X-ray analysis and from gravitational lensing 

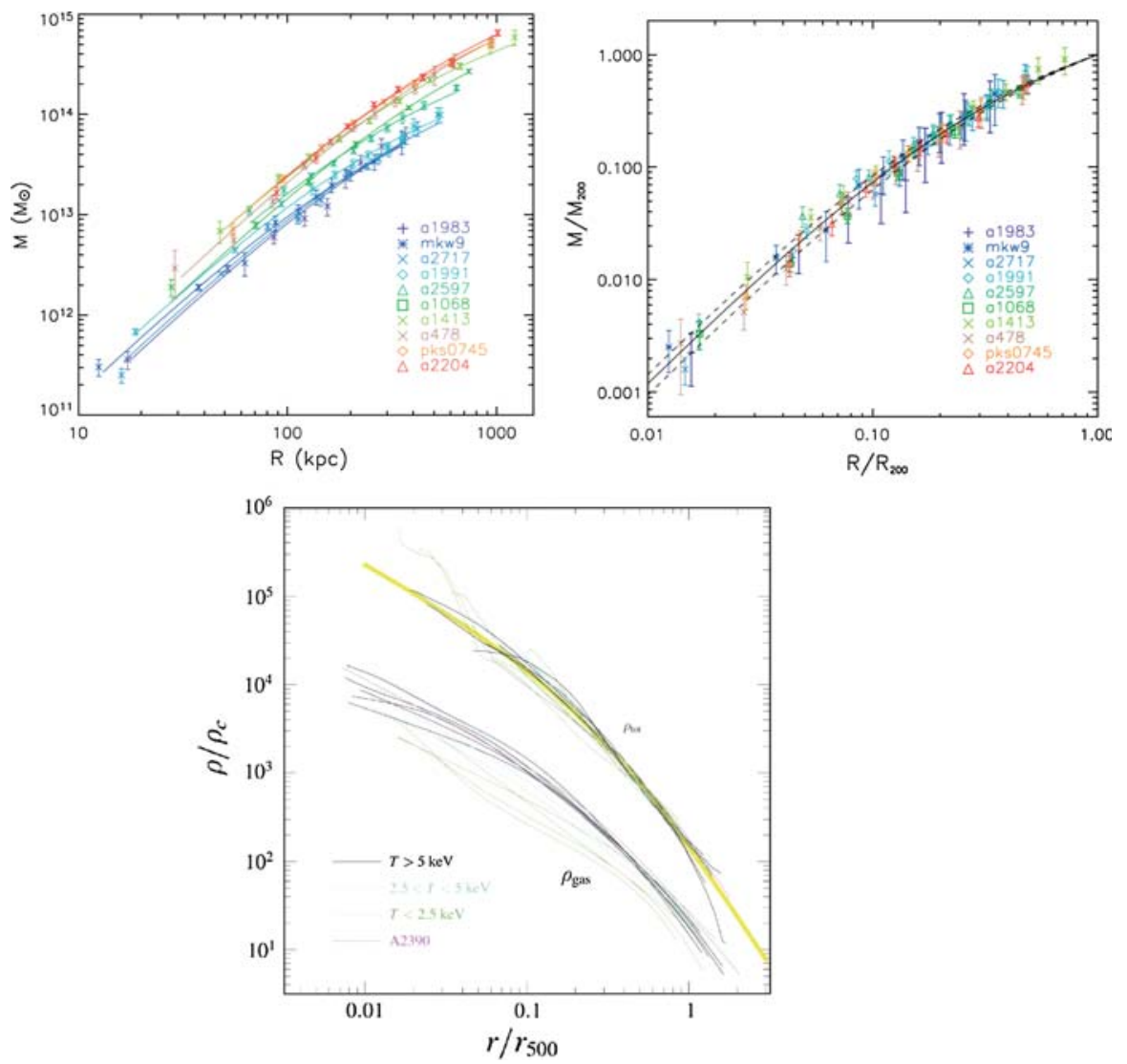

Fig. 11 Upper left Mass profiles for a sample of regular clusters with a range of temperatures derived from observations with XMM-Newton (Pointecouteau et al. 2005). Upper right Same mass profiles as in the left panel scaled in radius by $r_{200}$ and in mass by $M_{200}$, the mass at the radius of $r_{200}$. The solid line shows the fit of a NFW mass distribution model which provides a good description of the data. Lower panel Scaled gas density profiles and scaled total density profiles for a sample of galaxy clusters observed with Chandra (Vikhlinin et al. 2006). The yellow line shows the best fitting NFW-model for the total density profile

studies (Zhang et al. 2008; Mahdavi et al. 2008) showing that the X-ray mass determination provides a much smaller individual uncertainty. We also have first indications that the lensing masses are generally higher by about $12( \pm 15) \%$ which may partly reflect the above mentioned temperature bias and additional unaccounted turbulent pressure of the ICM if we assume that the lensing masses are essentially unbiased. This mass calibration by combining several methods will constitute an important effort in the coming years.

\subsection{Fossil record of structure and galaxy formation in the ICM thermal structure}

The temperature distribution in the ICM is also the key to the characterization of the thermal history and thermal structure, most conveniently expressed by the entropy structure of the cluster's ICM. The definition of "entropy", $S$, as used in the 
astrophysical literature of galaxy clusters deviates from the general definition in physics:

$$
S=\frac{k_{B} T_{X}}{n_{e}^{2 / 3}} .
$$

This definition is related to the general definition of entropy, $s$, by $s=k_{B}$ $\ln \left(S^{3 / 2}\left(\mu m_{p}\right)^{5 / 2}\right)+s_{0}$ (Voit 2005). The $S$ value can be seen as a parameter to label adiabates, and thus $S$ stays fixed in any hydrodynamic evolution of the ICM in which all processes are adiabatic; $S$ increases, e.g. in shock waves or with dissipation of turbulent motion and decreases with radiative cooling.

The most important application of studying the entropy structure in galaxy clusters is the distinction between heating by gravitational and non-gravitational processes. The term gravitational processes refers here to heat that comes from conversion of potential to heat energy in the formation of structure and the collapse of clusters. This form of heat is determined by structure formation processes that are mainly driven by the dark matter in the Universe. Non-gravitational processes are connected to energy input into the ICM in the form of star formation driven galactic winds (which at the same time lead to chemical enrichment of the ICM as discussed in Sect. 5) and by AGN providing energy input via jets of relativistic plasma.

The way to distinguish the two forms of heating is the study of scaling relations of thermal cluster properties. The gravitational heating of the ICM during cluster formation is proportional to the depth of the gravitational potential well of the cluster and thus the entropy gain is proportional to the ICM temperature. Heating processes connected to feedback from the galaxy population are expected to be related (proportional) to the total stellar mass in the cluster. Assuming to first order a constant stellar to gas mass ratio of the baryonic cluster component, the amount of non-gravitational energy input per unit gas mass is than also to first order constant. Therefore in this most simple consideration the gravitational entropy contribution is proportional to the virial and ICM temperature, while the non-gravitational entropy contribution is an additive constant. The evidence for non-gravitational entropy characterized in this way was provided by Ponman et al. (1999) as illustrated in Fig. 12 (see also David et al. (1996) who showed with ROSAT observations of cooler systems that groups have flatter entropy profiles than clusters). The results imply a so-called entropy floor of about $135 \mathrm{keV} \mathrm{cm}^{2}$ (Ponman et al. 1999; Lloyd-Davies et al. 2000). More modern results do not show the sudden appearance of a step, but rather a slope of the entropy-temperature relation which is shallower than the slope of 1 expected from purely gravitational models: instead of the gravitational slope of $S\left(r_{\text {scaled }}\right) \propto T$, relations around $\propto T^{2 / 3}$ are found. Purely gravitational models of smooth and cold accretion of gas into the cluster (e.g. Tozzi and Norman 2001) predict entropy profiles in clusters of the form $S(r) \propto r^{1.1}$. Observations show profiles which can be as steep as this prediction for the most massive systems, but they are shallower for smaller clusters, which is again a manifestation of the fact that non-gravitational processes have a larger effect on systems with a smaller mass and thus a shallower gravitational potential. As a representative result we show the study of Pratt (2006) involving 10 relaxed appearing clusters (Fig. 13), which illustrates the radial and ICM temperature scaling behavior of 
Fig. 12 Entropy in groups and clusters measured at a fiducial radius of $0.1 r_{\text {virial }}$ as a function of the system's ICM temperature (Ponman et al. 1999). It was inferred from this plot that the entropy appears to converge at low $T_{X}$ to a characteristic floor value of $\sim 100 h^{-1 / 3} \mathrm{keV} \mathrm{cm}^{2}$
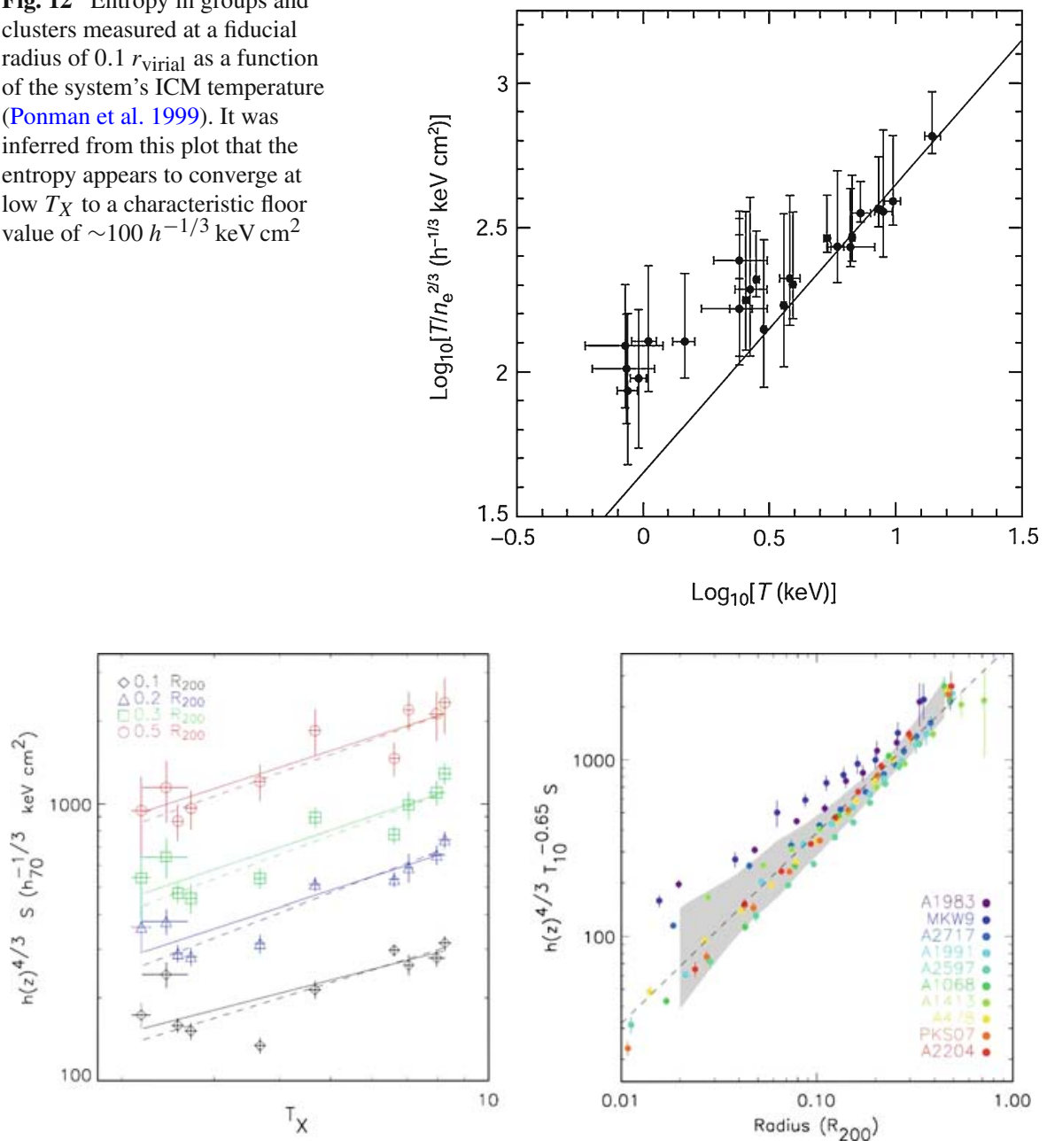

Fig. 13 Left The entropy-temperature relation for 10 regular galaxy clusters determined at four different fiducial radii (Pratt 2006). The slopes of the relations as determined from the orthogonal BCES method (Akritas and Bershady 1996) are for increasing radii: $0.49 \pm 0.15,0.62 \pm 0.11,0.64 \pm 0.11$, and 0.62 \pm 0.08 . Right Scaled radial entropy profiles for the same 10 clusters. The radius is scaled by $r_{200}$. The entropy, $S$, has been scaled with an entropy-temperature relation proportional to $T^{0.65}$ using the global cluster temperature. The grey shaded area corresponds to the $1 \sigma$ standard deviation. The dashed line shows radial dependence $S \propto r^{1.08}$ (Pratt 2006)

the cluster ICM entropy. The diagnostics of entropy profiles has now been pursued in detail for larger cluster samples by Cavagnolo et al. (2009) and representative cluster samples by Pratt et al. (2009, in preparation) providing a more detailed picture, the proper interpretation of which has to be assisted by simulations.

Theoretical modeling to explain the quantitative entropy scaling behavior involves "preheating" (early entropy increase of the intergalactic medium due to star formation before the formation of the cluster), cooling and condensing of the low entropy material which increases $S$ in the remaining gas phase, and feedback heating processes 
in the cluster. A complicated balance of all these processes seems to be necessary to reproduce the entropy profiles, the scaling relations, and the amount of baryons that are converted into stars in the cluster volume and efforts to obtain a completely consistent and satisfying model are still ongoing (e.g. Borgani et al. 2004, 2005; Voit et al. 2003; Voit 2005; McCarthy et al. 2008).

\subsection{At and beyond the virial radius}

The low, stable, and well understood instrumental and particle background properties of the Suzaku satellite allowed to perform the first pioneering studies of clusters out to and beyond their virial radius. Reiprich et al. (2008) determined the temperature profile for the rich, massive, cooling core cluster Abell 2204 from $\sim 10$ to 1,800 kpc, close to the estimated $r_{200}$. They find that the temperature profile between 0.3 and $1.0 r_{200}$ is consistent with a drop of 0.6 , as predicted by simulations. The first temperature, density, and entropy profiles beyond $r_{200}$ were reported for another rich, massive cooling core cluster PKS 0745-191 (George et al. 2009). Between 0.3 and $1 r_{200}$ they measure an average temperature drop of $\sim 70 \%$. They find that near to the virial radius the observed entropy profile is lower than that expected for the heating by gravitational collapse. Figure 14 shows the observed profiles in four directions and the average profiles. The dotted curve at the bottom shows the expected entropy profile for heating by gravitational collapse, $S \propto r^{1.1}$, and the vertical dashed line shows the estimated $r_{200}$. The fall of entropy beyond $r_{200}$ in the NW direction is interpreted by the authors as evidence for an accretion shock from cooler material falling on the cluster along a filament.

$\mathrm{X}$-ray emission from hot plasma associated with a filament connecting two massive clusters of galaxies, Abell 222 and Abell 223, was recently detected using XMMNewton (Werner et al. 2008). The detection of the tenuous gas permeating the filament was possible because of its favorable orientation approximately along our line-ofsight. The temperature of the detected gas is $k T=0.91 \pm 0.25 \mathrm{keV}$ and assuming that the length of the filament along the line-of-sight is $l=15 \mathrm{Mpc}$ its baryon density is $\approx 3.4 \times 10^{-5} \mathrm{~cm}^{-3}$, which corresponds to a baryon over-density of $\approx 150$. The entropy of the gas in the filament is $S \approx 870 \mathrm{keV} \mathrm{cm}^{2} l^{1 / 3}$, which suggests strong preheating. We note that if the detected emission would be associated with the outer region of radially asymmetric clusters (deriving the gas density by means of the usual geometric deprojection), the corresponding entropy of $\approx 420 \mathrm{keV} \mathrm{cm}^{2}$ would be much lower than that expected for the virialized cluster gas $\left(\approx 1,000 \mathrm{keV} \mathrm{cm}^{2}\right)$ which has already passed through an accretion shock.

\subsection{Diagnostics of cluster mergers}

Galaxy clusters grow throughout the recent history of our Universe by accreting matter from their surroundings, preferentially by clumpy accretion from the cosmic network matter filaments in the intersections of which they are embedded (e.g. Braglia et al. 2007). From time to time major merger events happen in this accretion process, where two larger systems are attracted to each other and merge violently. These cluster 


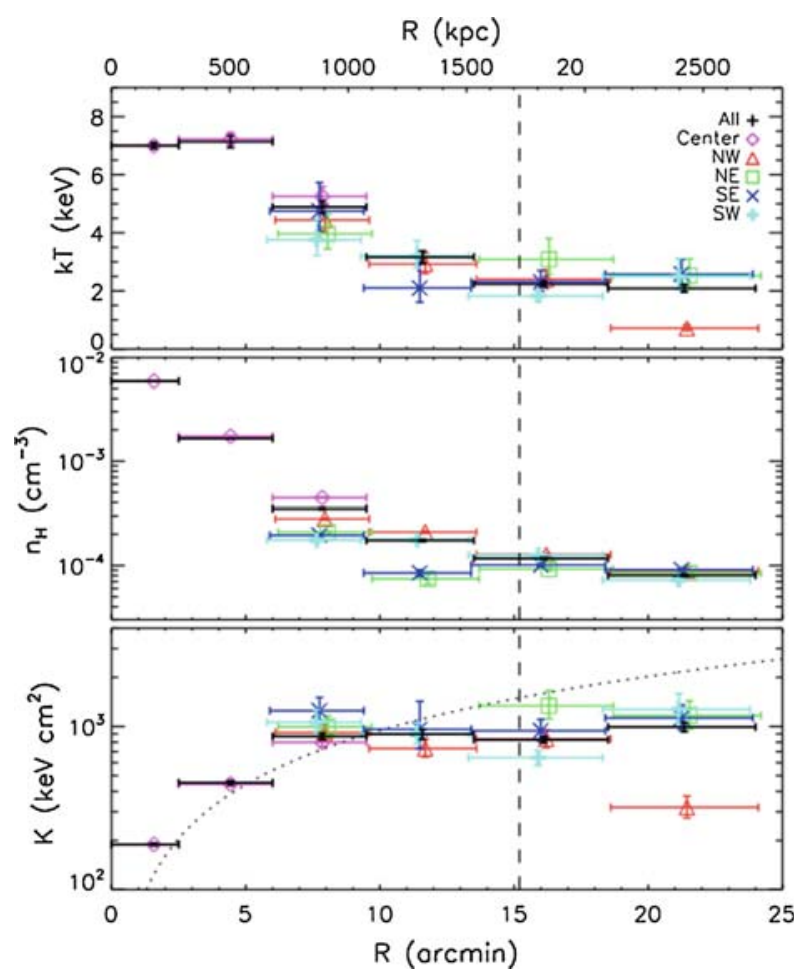

Fig. 14 Temperature, density and entropy profiles of PKS 0745-191 determined by Suzaku out to and beyond the virial radius in each direction. The dotted curve at the bottom shows the expected entropy profile for heating by gravitational collapse $S \propto r^{1.1}$. The vertical dashed line shows the estimate of $r_{200}$ (George et al. 2009)

mergers have always been very attractive study objects (see, e.g. Feretti et al. 2002, for a review). The thermal structure of the ICM again offers good perspectives to unveil the merger configuration and its history as well as to understand the heating processes of the ICM resulting from the energy release of the merger.

We will illustrate the diagnostic potential of X-ray spectroscopy in these studies by concentrating here mostly on the example of the most dramatic merging system, 1E0657-56. But before we describe an earlier result obtained with XMM-Newton for the diagnostics of the off-axis merger in the cluster A3921 shown in Fig. 15 by Belsole et al. (2005). The temperature map has been obtained by applying the multiscale spectro-imaging technique based on a wavelet analysis described by Bourdin et al. (2004). The general result of this analysis was subsequently confirmed by spectroscopy of the regions highlighted in the temperature map. While the general gas temperature of the undisturbed Eastern region of the main cluster has a temperature around $4.9 \mathrm{keV}$, a hot, bar like region with an extent of about $160 \times 280 \mathrm{kpc}$ is observed in the highly distorted North-Western side which has been found to have a temperature around $7.25 \mathrm{keV}$. The signatures have been interpreted as an off-axis merger (Belsole et al. 2005). The observed features have been found to be similar to those in the simulations of an off axis merger with a mass ratio of 1:3 an impact parameter of $b=5 r_{s}$ 


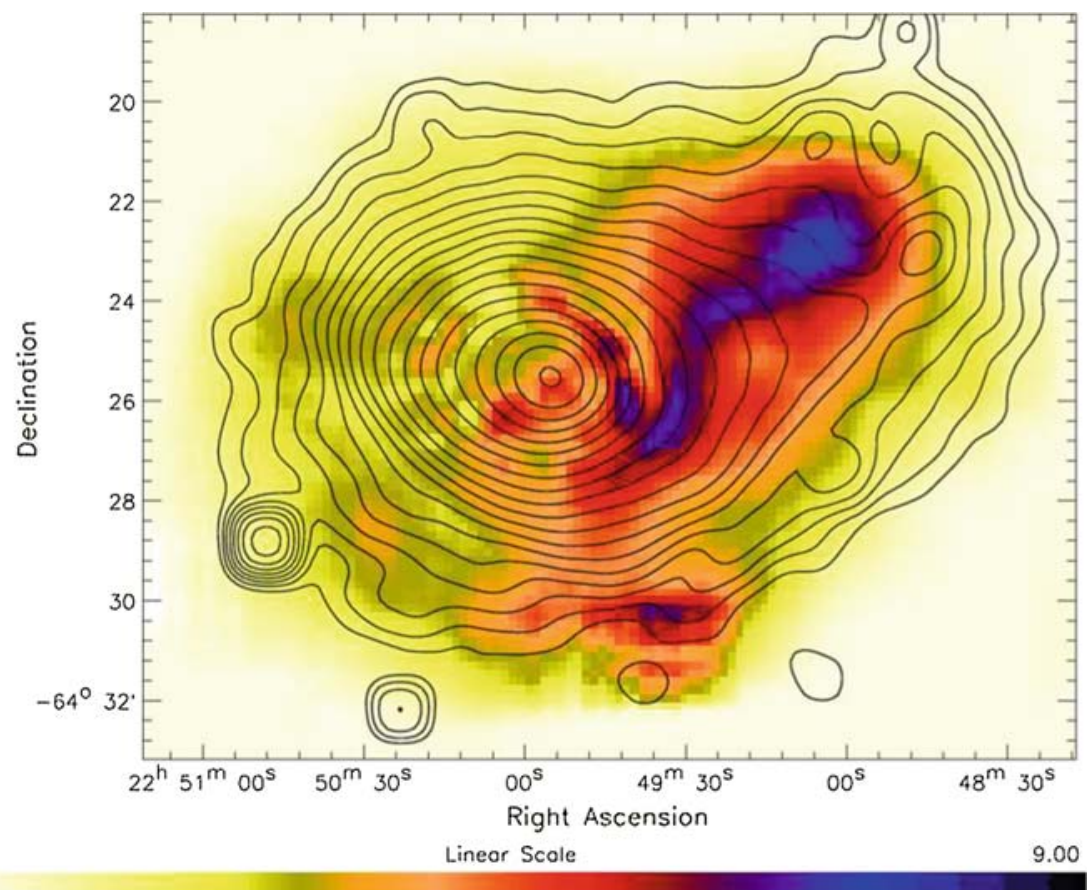

Fig. 15 Temperature map of the merging cluster A3921 by Belsole et al. (2005). The temperature map has been produced from XMM-Newton observations obtained with the detectors EMOS1 and EMOS2 with the multi-scale spectro-imaging technique of Bourdin et al. (2004). The barlike hot region to the NE is interpretated as the signature of an off-axis merger at a stage short after the first close encounter similar to features observed in simulations (Belsole et al. 2005; Ricker and Sarazin 2001). Superposed to the temperature map are the contour lines of the surface brightness distribution in the $0.3-10 \mathrm{keV}$ band which was adaptively smoothed. The contours are spaced logarithmicly

(where $r_{s}$ is the scale radius of the NFW profile describing the density distribution of the cluster) and a time of less than about $1 \mathrm{Gyr}$ after the X-ray luminosity reaches its peak as shown by Ricker and Sarazin (2001) in their Fig. 7. This system seems to show the typical appearance of an intermediate stage off-axis merger similar to other cases (e.g. Reiprich et al. 2004).

The most detailed and interesting data set on a merging cluster system is that of 1 E0657-56 at a redshift of $z=0.297$. This cluster was observed in a very deep observation with Chandra with an exposure of $500 \mathrm{ks}$ (Markevitch 2006) and also with XMM-Newton (Zhang et al. 2008; Finoguenov et al. 1996). As a first exercise to get an overview on the thermodynamic structure of the cluster ICM, it has become standard for such deep, high photon statistic observations to produce maps of the temperature and density distributions of the ICM and to infer the pressure and entropy distribution from these quantities. Figure 16 shows such maps produced by (Million and Allen 2008). The temperature is determined from spectroscopy of about 100 image pixels, each containing at least 3,000 photons. The binning of these pixels has been guided by regions of similar surface brightness (Sanders 2006). The ICM density is derived from the surface brightness distribution by assuming a certain geometry to deproject 

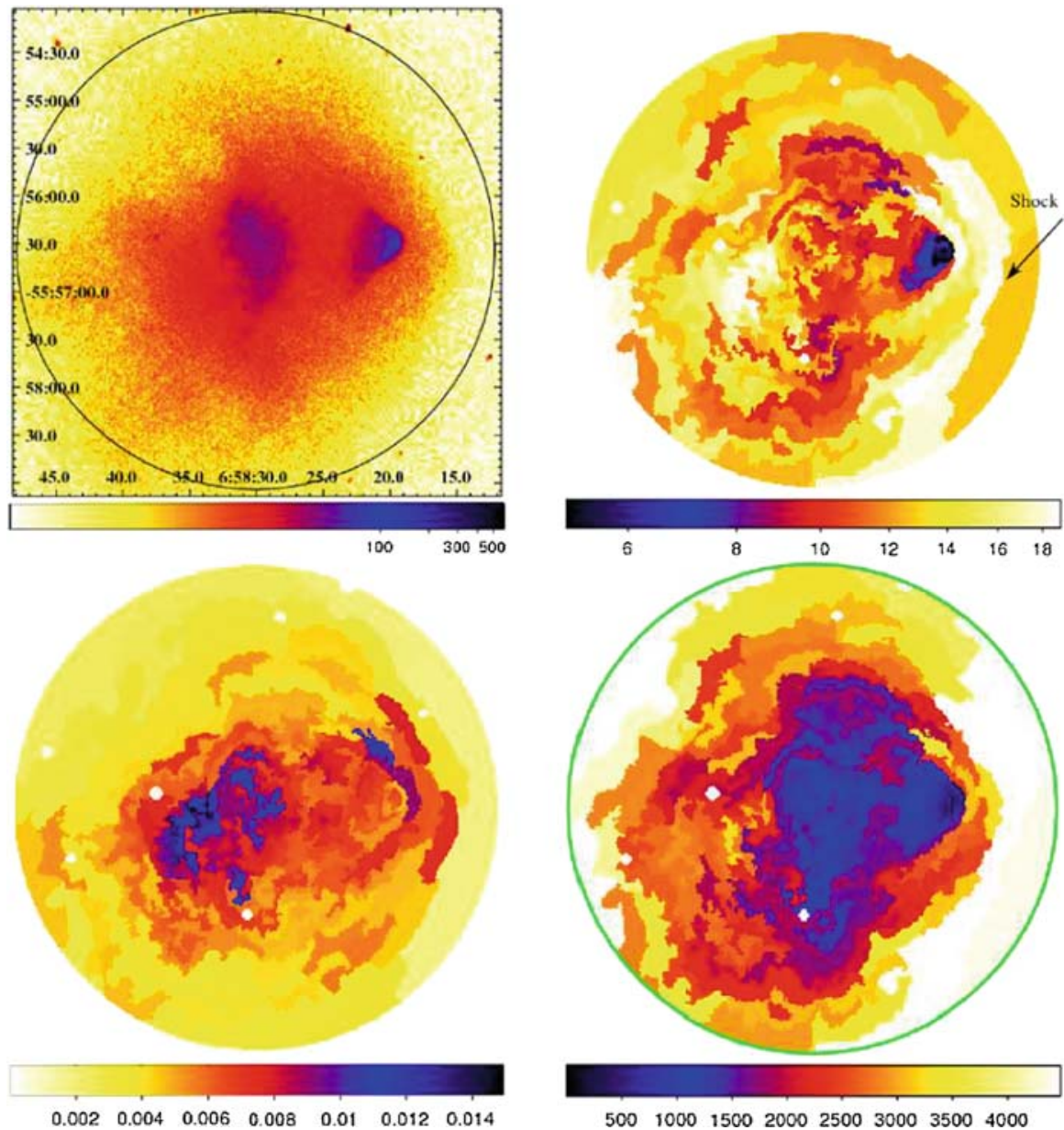

Fig. 16 Thermodynamic maps for the ICM of the "bullet cluster", 1E0657-56 (Million and Allen 2008) produced from a $500 \mathrm{ks}$ observation of this cluster with Chandra. The panels show the X-ray surface brightness in the $0.8-7 \mathrm{keV}$ band (upper left), the temperature, $k_{B} T$, in units of $\mathrm{keV}$ (upper right), the projected pressure in units of $\mathrm{keV} \mathrm{cm}^{-5 / 2} \operatorname{arcsec}^{-1}$ (lower left) and projected entropy in units of $\mathrm{keV} \mathrm{cm}^{5 / 3} \operatorname{arcsec}^{-1}$ (lower right). The shock front preceding the bullet is marked by an arrow in the temperature map

the emission distribution along the line-of-sight. Although the so derived temperature in the temperature map is a projected quantity, it still gives a good impression about the temperature in the central bin in the line-of-sight because of the large weight of the innermost bin due to the square density dependence of the emissivity and the steep density profile. From these maps of approximate temperature and density distribution in a cross section of the cluster, the approximate distribution of pressure, $P=n k_{B} T_{x}$, and entropy, $S=T / n^{2 / 3}$, can be constructed. The surface brightness image in Fig. 16 shows a disturbed larger cluster component and a compact, cone like structure to the West. The latteris identified with a compact subcluster, flying through the main cluster at high relative velocity, for which optical images provide further evidence. This component has been named "the bullet" from which the popular name of the whole 

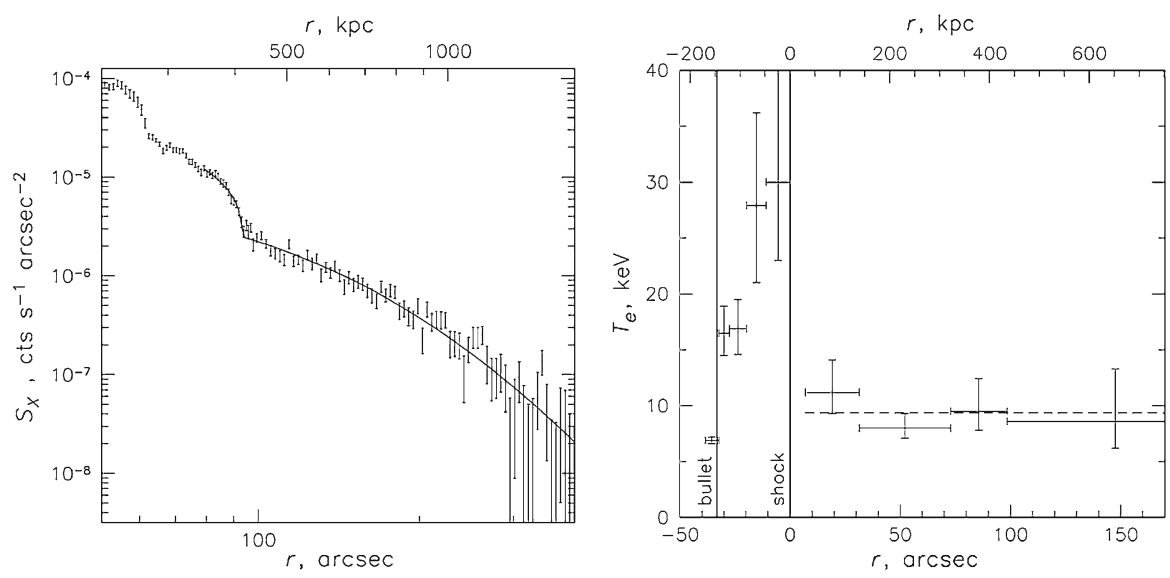

Fig. 17 Left Surface brightness profile across the shock front preceding the bullet (Markevitch et al. 2002). The solid line shows the best fit model for the three-dimensional density distribution with a sharp spherical discontinuity at the shock. Right Projected temperature profile in a narrow sector across the shock front. The vertical lines show the boundary of the bullet and the shock. The dashed line shows the average pre-shock temperature (Markevitch 2006). Both data sets have been derived from deep Chandra observations

system, "the bullet cluster", originates. In the temperature map the bullet shows up as a CC which also has the lowest entropy in the entropy map. The Mach cone like shape of the bullet suggest that it may fly with supersonic velocity. In the temperature map we observe that the region in front of the bullet has a strongly enhanced temperature that goes hand in hand with high pressure and elevated entropy. This is the signature of a region heated by a shock preceding and being detached from the bullet. The high temperature/entropy region shows a sharp edge at the shock. In the pressure map the region of the bullet shows little enhancement (apart from the narrow band of shock compressed ICM), while the highest pressure values are found in the center of the overall system structure. Thus, despite the disturbances, the pressure maximum is most probably still indicating the region of the deepest gravitational potential.

To obtain more quantitative information about the nature of the shock in front of the bullet, (Markevitch et al. 2002) has studied the temperature and density distribution around the region of the shocked ICM in more detail. Figure 17 shows two sharp edges in the surface brightness profile in the left panel. The inner bump is the contact discontinuity that separates the bullet from the shock heated ICM, the second bump at $90^{\prime \prime}$ is the shock. From a density model that fits the projected surface brightness one infers a density jump that corresponds to Mach number $3 \pm 0.4$ shock. The corresponding temperature jump as derived from the projected spectra, is shown in the right panel. The relatively high Mach number implies a relative velocity of the bullet and the ICM of about $4,700 \mathrm{~km} / \mathrm{s}$. For an explanation of this high velocity see recent simulations that try to reconstruct the merger configuration of 1E0657-56 and recover such high velocities which are partly boosted by the accretion inflow of ICM at the position of the bullet (Springel and Farrar 2007; Mastropietro and Burkert 2008). In Sect. 6.2 we discuss the use of the observed shock structure in this cluster to study the processes controlling the thermalization of the plasma behind the shock. 
So far only two more cases of clear shock signatures in merging clusters are known: A 520 (Markevitch et al. 2005; Markevitch and Vikhlinin 2007); A2219 (Million and Allen 2008).

Another type of interesting structures in the ICM are the so called cold fronts, which also show up in the surface brightness images of clusters as sharp surface brightness discontinuities. But thanks to the spectroscopically determined temperatures one can show that the pressure across the cold fronts is continuous, such that they are boundaries between colder denser plasma and a more tenuous, hotter environment. Markevitch and Vikhlinin (2007) provide a nice recent review about the X-ray observations and the physics of cold fronts and ICM shocks.

\subsection{Observational studies of turbulence of the ICM}

Galaxy cluster mergers are expected to induce turbulent motions into the ICM (Sunyaev et al. 2003). An investigation of the presence of stochastic turbulence has been performed by means of a spectral reduction of deep XMM-Newton observations of the Coma galaxy cluster by Schuecker et al. (2004). The turbulence fluctuation spectrum was studied in the projected pressure map of the ICM in the central region of the Coma cluster. The Coma cluster has a very flat appearance, characterized by a very large core radius of the X-ray surface brightness of $\sim 400 \mathrm{kpc}$ (Briel et al. 1992). This enables us to treat the configuration of the central region of Coma in the first approximation as a slab geometry, with corrections to the power spectrum applied later. The analysis of the fluctuation spectrum testing for a turbulent power law spectrum was performed, with the pressure rather than with density or temperature fluctuations, not to confuse the turbulent fluctuations with static entropy fluctuations in pressure equilibrium, which would be characterized by contact discontinuities. In fact, Fig. 19 (left) shows that turbulent pressure fluctuations dominate also the density fluctuations rather than contact discontinuities.

The map of the projected pressure distribution in the center of the Coma ICM as shown in Fig. 18 was obtained by calculating the gas density from the X-ray surface brightness (with an assumed depth of the ICM in the line of sight) and deriving the temperature by a spectral analysis of the data in pixels of $20 \times 20 \operatorname{arcsec}^{2}$ and alternatively $40 \times 40 \operatorname{arcsec}^{2}$, yielding the pressure by means of the ideal gas equation of state. We see the pressure fluctuations clearly in Fig. 18. A Fourier analysis of these fluctuations seen in projection results in the power spectrum shown in Fig. 19 (right). This spectrum has been corrected for the contribution of Poisson noise and the overall shape of the ICM surface brightness in Coma. The observed power spectrum is characterized by a shape very close to a power law. In Fig. 19 we show how different 3-dimensional power law functions compare to the observed spectrum if they are projected in the same way as the observed spectrum. The observations lay in between a power law exponent of $5 / 3$ and $7 / 3$. An exponent of $7 / 3$ is the one expected for the pressure fluctuation spectrum of the classical prediction of Kolmogorov (1941) and Oboukhov (1941). The original work by Kolmogorov was developed for an incompressible fluid, and in the ICM also magnetic fields play a not fully quantified role, and therefore the case of the cluster ICM is not easily comparable to the classical picture. Nevertheless, 


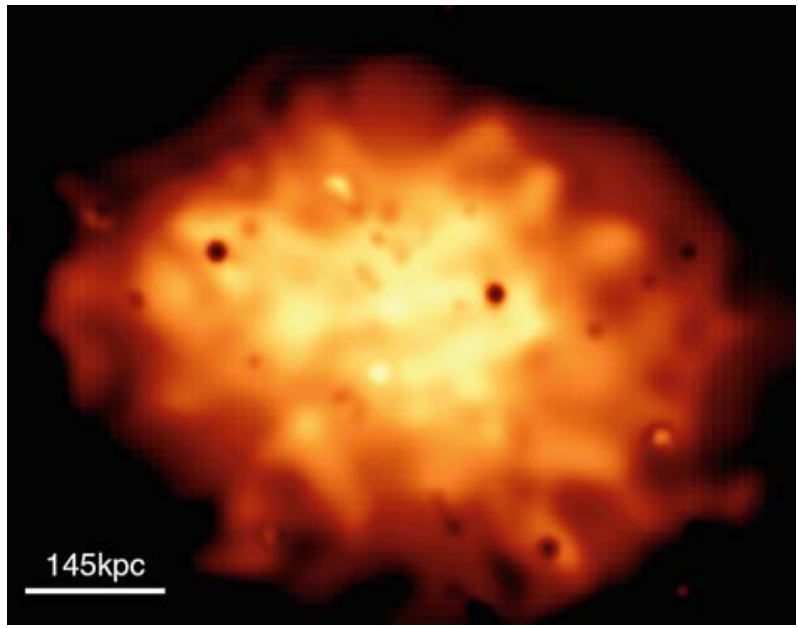

Fig. 18 Map of the projected pressure distribution of the ICM in the central region of the Coma galaxy cluster. The scale size of $145 \mathrm{kpc}$ corresponds to the largest turbulent eddies traced by the observed pressure fluctuation power spectrum (Schuecker et al. 2004)
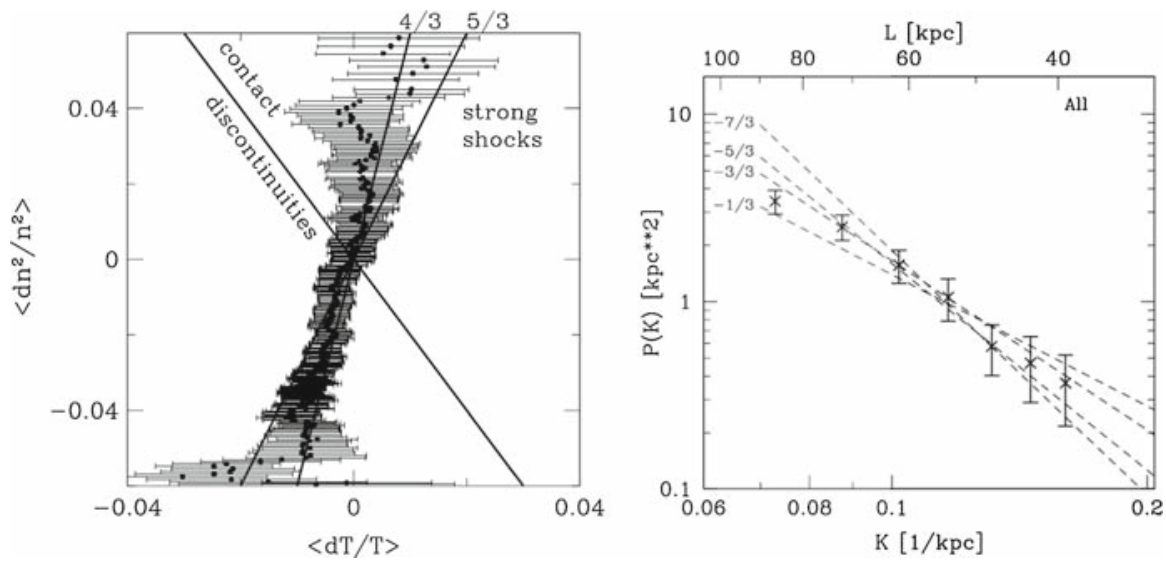

Fig. 19 Left Correlation of the relative fluctuations in squared density, $n^{2}$, and temperature, $T$, and their $1 \sigma$ error bars. The solid lines give the adiabatic exponents of $\gamma=5 / 3$ (ideal gas) and $\gamma=4 / 3$. The third line shows the anticorrelation of density and pressure expected for contact discontinuities. The positive correlation not far from an ideal gas exponent indicates that adiabatic pressure fluctuations, as expected for the ram pressure effects of turbulent motions dominate also the density and temperature fluctuation spectrum. Right Resulting shot noise subtracted, projected power spectrum of the pressure fluctuations observed in the central region of the Coma galaxy cluster (with $1 \sigma$ errors). Also shown are power spectra for different power law shapes projected in an analogous way as the observed spectrum. The model power spectra are labeled with the exponents of the non-projected 3-dimensional power spectra. A Kolmogorov-Obuchov spectrum with an exponent of $n=-7 / 3$ is not far from the results, which lay in between $n=5 / 3$ and $n=7 / 3$ (Schuecker et al. 2004)

a very similar dimensional consideration of turbulence (see, e.g. Landau and Lifshitz, vol. VI) is surely applicable to the cluster ICM where most importantly a scale free spectrum is expected between the driving and dissipation scale. The observed signature 
of a nearly scale free power law power spectrum is thus a very interesting result. The observations have therefore been interpreted in the way that a nearly classical turbulence configuration has been established in the Coma cluster ICM (Schuecker et al. 2004). This can most probably be explained by the fact that Coma is generally believed to be a post-merger cluster (White et al. 1993).

Important information on the amount of turbulence and turbulent pressure support can also be obtained by measuring the level of resonant scattering in emission lines observed in the ICM. The ICM is generally assumed to be optically thin. This is certainly true for most of the emitted X-ray photons, but for the strongest resonance lines the ICM can be moderately optically thick (Gilfanov et al. 1987). What happens is that for strong resonance lines the transition probabilities are large, photons get absorbed, but since the time between the absorption and emission is extremely short they get very quickly reemitted into a different direction. Because of the very short time between the absorption and the emission of the photon the process can be effectively regarded as scattering. Gilfanov et al. (1987) pointed out that because the optical depth of the resonance line depends on the characteristic velocity of small-scale gas motions, measurements of this optical depth give important information about the turbulent velocities in the hot plasma.

The first constraints on turbulent velocities using resonant scattering were obtained by Churazov et al. (2004), who used XMM-Newton EPIC data to compare the relative fluxes of the He-like $\mathrm{Fe} \mathrm{K}_{\alpha}$ and $\mathrm{Fe} \mathrm{K}_{\beta}$ lines in the core and in an annulus around the core of the Perseus cluster. Since the $\mathrm{Fe} \mathrm{K}_{\alpha}$ at $6.7 \mathrm{keV}$ has a much larger optical depth than the $\mathrm{K}_{\beta}$ line a difference in their ratios in the two spatial regions with different column densities would be an evidence for resonance scattering in the core of Perseus. Churazov et al. (2004) found no evidence for resonance scattering in Perseus, indicating that differential gas motions on scales smaller than $\sim 100 \mathrm{kpc}$ in the core of the cluster must have a range of velocities of at least half of the sound speed. Independently, Gastaldello and Molendi (2004) reached similar conclusions.

The first unambiguous evidence for resonant scattering was found using highresolution spectra of the hot halo around the giant elliptical galaxy NGC 4636 obtained by XMM-Newton RGS (Xu et al. 2002). The plasma with temperatures below $0.9 \mathrm{keV}$ observed in elliptical galaxies, groups of galaxies, and in the CCs of some clusters emits three strong Fe XVII lines. While the line at $15.01 \AA$ has a very strong oscillator strength and is expected to be optically thick, the blend of lines at 17.05 and 17.1 A have negligible optical depths. The radial profile of the ratios of these two lines (Fe XVII 17.1 / Fe XVII 15.0 ̊) derived using RGS shows a clear gradient with the peak in the center of the galaxy NGC 4636, proving that many of the $15.01 \AA$ photons get scattered before exiting its core (see Fig. $20 \mathrm{Xu}$ et al. 2002).

Following up the work by Xu et al. (2002); Werner et al. (2009) analyzed the $X M M-N e w t o n$ RGS data of five nearby bright elliptical galaxies and found that the FeXVII lines in the cores of four galaxies show evidence for resonance scattering in the innermost region. The data for NGC 4636 in particular allowed the effects of resonant scattering to be studied in detail. Werner et al. (2009) used deprojected density and temperature profiles obtained by Chandra to model the radial intensity profiles of the strongest resonance lines, accounting for the effects of resonant scattering, for different values of the characteristic turbulent velocity. Comparing the model to the 


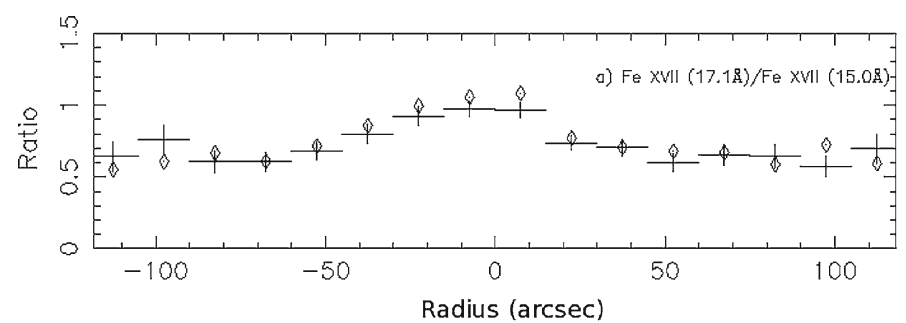

Fig. 20 Ratio of the $2 p-3 s$ lines of Fe XVII at 17.05 and $17.10 \AA$ to the $2 p-3 d$ line of the same ion at $15.01 \AA$. The ratio has a significant peak at the center of the galaxy, NGC 4636. Since the lines originate from the same ion, the ratio cannot be due to a spatial abundance gradient. Given the narrow temperature range and the weak dependence of the line ratio on temperature it cannot be due to a temperature gradient either. This trend is the best evidence so far for resonant scattering of the line at $15.01 \AA$ in a hot galactic halo

data they found that the isotropic turbulent velocities on spatial scales smaller than $\approx 1 \mathrm{kpc}$ are less than $100 \mathrm{~km} \mathrm{~s}^{-1}$ and the turbulent pressure support in the galaxy core is smaller than $5 \%$ of the thermal pressure at the $90 \%$ confidence level, and less than $20 \%$ at $95 \%$ confidence. Note that the spatial scales of turbulence probed in the cores of elliptical galaxies by RGS are much smaller than those probed in Perseus (Churazov et al. 2004) or Coma (Schuecker et al. 2004).

The FeXVII lines are the most sensitive probes of turbulence using resonant scattering, but because they are present only at temperatures less than $\sim 1 \mathrm{keV}$ they cannot be used to probe turbulence in the much hotter clusters of galaxies. High-resolution spectra obtained by X-ray calorimeters on the future satellites like Astro- $H$ and IXO will allow us to probe turbulence also in higher mass systems and at larger radii (see Sect. 7).

\section{AGN heated cluster CCs}

The temperature profiles shown in the right hand panel of Fig. 10 reveal two types of clusters: those with temperature profiles falling toward the center are called CC clusters; and clusters with no central drop of temperature are called NCC clusters. While the former also have high densities in their cores which implies short cooling times for the central ICM, typically one or two orders of magnitude smaller than the Hubble time, the NCC clusters have usually central cooling times exceeding the Hubble time. This observation implies that the ICM plasma should cool and condense in the CC clusters in the absence of any heat source, which could balance the cooling. Theoretical considerations focussed on the consequences of cooling without heating lead to the so-called "cooling flow" scenario (Fabian and Nulsen 1977; Fabian 1994), which predicts mass deposition in the centers of some clusters at a rate of up to hundreds to thousands of solar masses a year. In general more than half of the clusters in the nearby Universe should have cooling flows in this model (Peres et al. 1998), where the exact fractional number depends to some degree on the definition of a cooling flow.

Interestingly, in particular in the context of this review, the cooling flow model leads to the prediction of very specific and testable spectral signatures of a steady 


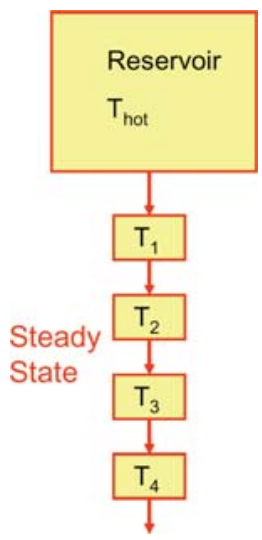

Fig. 21 Sketch of the temperature evolution of a classical cooling flow model, with a reservoir of only slowly cooling hot gas at low density and a steady cooling of the fraction of gas with a mass cooling rate, $\dot{M}$, down to lower temperatures. The spectrum of this cooling flow is then determined by the integral of the spectra of the transient temperature phases, where each temperature phase contribution is fixed by the amount of heat that has to be radiated in the given temperature interval to cool an amount of ICM with mass $\dot{M}$ (see equations in the text)

state cooling flow. Looking at the plasma distribution in this scenario in temperature space, we have a reservoir of hot plasma and a steady cooling of some plasma with a mass deposition rate $\dot{M}$, as sketched in Fig. 21. This simplified picture of a reservoir of hot gas and a constant fraction of gas cooling is motivated by the fact that cooling is accelerating with decreasing temperature because the plasma is getting denser while it is cooling in pressure equilibrium and the cooling radiation power is proportional to the gas density squared. For the part of the plasma that is steadily cooling to lower temperature, each temperature interval has to contribute to the cooling radiation with a power equal to the enthalpy change of the plasma that is cooling across this temperature interval:

$$
L_{x} \mathrm{~d} T=\frac{\mathrm{d}}{\mathrm{d} t} \text { Enthalpy } \mathrm{d} T=\frac{5 k_{b} \mathrm{~d} T}{2 \mu m_{p}} \dot{M} .
$$

To calculate the expected spectrum we have to weight this power by the ratio of the emission at a certain frequency to the bolometric emission:

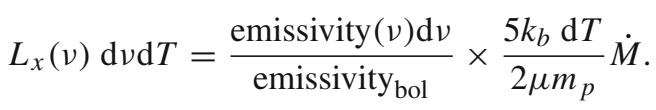

To obtain the total spectrum radiated by all steady cooling temperature phases we have to integrate the above equation over $\mathrm{d} T$ : 


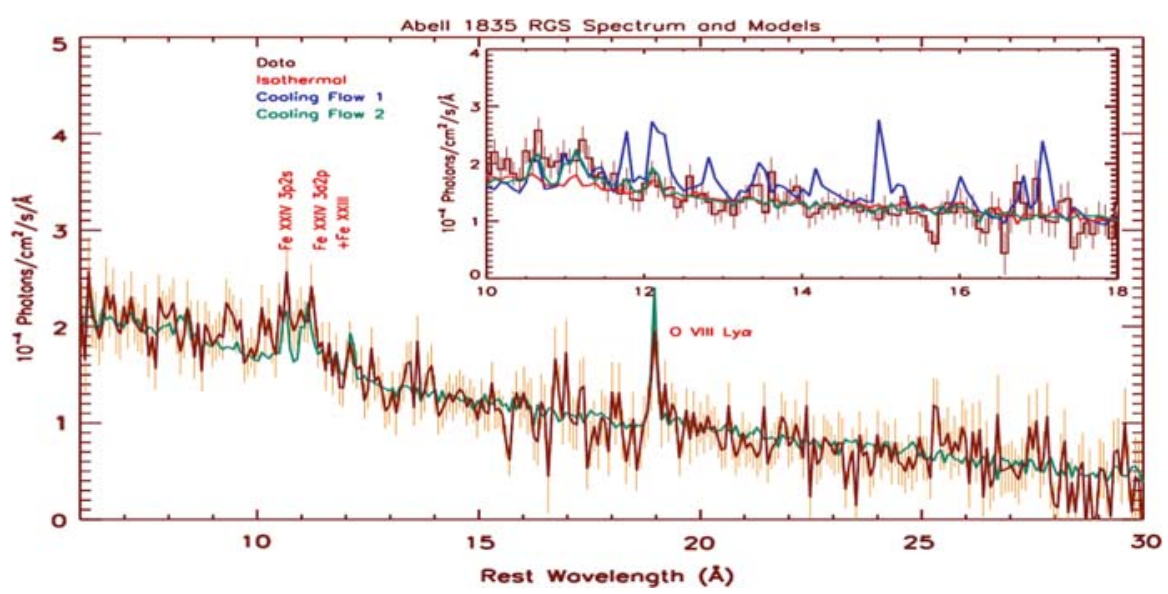

Fig. $22 X M M$-Newton RGS spectrum of the central region of the prominent cool core cluster, A1835 compared to model predictions (Peterson et al. 2001). The red line shows a spectral model for an isothermal temperature of $8.2 \mathrm{keV}$. The blue line shows the expectation for the classical cooling flow and an ambient temperature of $8.2 \mathrm{keV}$, the green line shows a cooling flow model where temperatures below $2.7 \mathrm{keV}$ have been truncated. Clearly, some of the cooling flow predicted lines are missing from the observed spectrum

$$
L_{x}(v) \mathrm{d} v=\frac{5 k_{b} \mathrm{~d} T}{2 \mu m_{p}} \dot{M} \int_{T_{\text {cutoff }}}^{T_{\text {hot }}} \frac{\Lambda_{v}\left(T^{\prime}\right) \mathrm{d} v}{\Lambda_{\text {bol }}\left(T^{\prime}\right)} \mathrm{d} T^{\prime},
$$

where $\Lambda_{v}(T)$ and $\Lambda_{\text {bol }}(T)$ are the emissivity of the plasma (radiation power per unit emission measure) for radiation at frequency, $v$, and for bolometric radiation, respectively.

With the advent of high quality spectra provided by XMM-Newton and Chandra, the cooling flow paradigm could be tested by spectroscopy. First evidence that the cluster $\mathrm{CCs}$ are not consistent with the classical cooling flow model came from spectra analyzed with the Reflection Grating Spectrometers on XMM-Newton. Some of the CC clusters have such highly peaked surface brightness profiles, that they look almost like blurred point sources to the RGS (which operates analogously to slitless spectroscopy in optical astronomy). Therefore the RGS can obtain spectra with much higher resolution than provided by the imaging CCD devices of the XMM-Newton EPIC cameras for these very peaked CC regions. Figure 22 shows the first published RGS spectrum of one of the very strong CC clusters, A1835, at a redshift of $z=0.2523$ (Peterson et al. 2001). In Fig. 22 the observed XMM-Newton spectrum (dark red) is compared to the prediction for the cooling flow model with a mass deposition rate inferred from the total radiative emission in the cooling flow region determined from the X-ray images (blue curve). There are clearly some expected lines, notably those of Fe XVII, missing in the observations. A more detailed analysis of the spectrum shows that for an ambient ICM temperature of $8.2 \mathrm{keV}$, the central regions shows temperature phases down to about $2.7 \mathrm{keV}$, but the expected intermediate temperature phases below $2.7 \mathrm{keV}$ are absent in the spectrum. This was generally taken as the first evidence that there is 

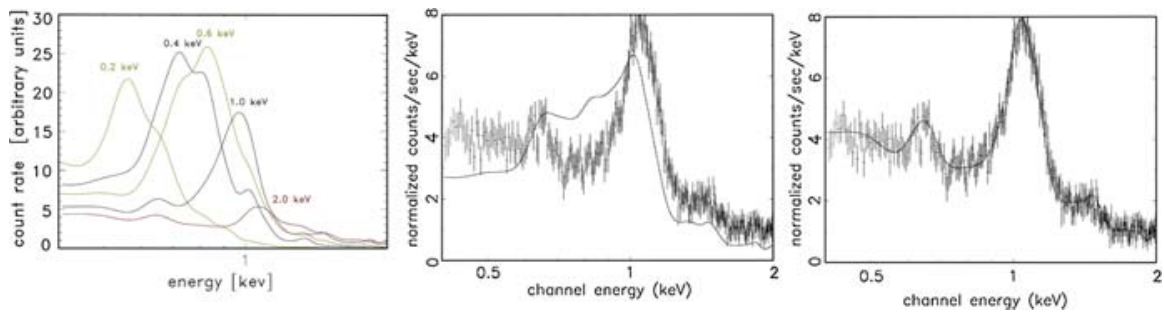

Fig. 23 Left Spectrum of hot plasma at various temperatures around the Fe L-shell line blend near $1 \mathrm{keV}$ as observed with the response function of the XMM-Newton EPIC pn instrument. This feature lends itself as a nice thermometer for the temperature range $0.2-2 \mathrm{keV}$. Middle Predicted X-ray spectrum around the Fe L-shell line blend for the cooling flow in the X-ray halo of M87 with a mass deposition rate of $\dot{M} \sim$ $1 \mathrm{M}_{\odot}$ year $^{-1}$ compared to the actual spectrum in the radial region $r=1-2 \operatorname{arcmin}$ as observed with the $X M M-N e w t o n$ EPIC pn instrument. The observations are clearly inconsistent with the predictions from the classical cooling flow model. Right Reproduction of the observed spectrum by assuming that the plasma in this region of the M87 halo occupies the narrow temperature interval of about $1.44-2 \mathrm{keV}$ (for more details see Böhringer et al. 2002)

something wrong with the classical cooling flow model. Since at the same time high angular resolution Chandra images showed signs of strong interaction of the central AGN with the ambient ICM in many CC regions (e.g. David et al. 2001; Nulsen et al. 2002; Fabian et al. 2005), the most probable solution to the absence of strong cooling was soon believed to be the heating of CC regions by the central AGN.

The spectral signature of the absence of cooling was not only obtained from XMMNewton RGS spectra. For example for the CC region in the X-ray halo of M87, the central dominant galaxy in the northern part of the Virgo cluster, clear evidence for an inconsistency of the spectral signatures with a classical cooling flow model could also be provided by results with the EPIC pn and MOS cameras of XMM-Newton. The most prominent feature that can be used for these diagnostics is the blend of Fe lines in the spectrum from transitions into the $\mathrm{L}$-shell at photon energies around $1 \mathrm{keV}$. Figure 23 shows how the spectral feature of this blend of Fe lines changes with temperature. The shift is caused by the changing degree of ionization of the Fe ions that contribute to the line blend. When the temperature is lowered more and more electrons in the Fe ions shield the charge of the nucleus decreasing the binding energy of the L-shell electrons. As illustrated in Fig. 23 this spectral feature can be used as a sensitive thermometer in the temperature range of $0.2-2 \mathrm{keV}$ (Böhringer et al. 2002). The middle panel in Fig. 23 shows the spectrum implied for the broad temperature distribution expected for the cooling flow in M87 (in the radial range 1-2 arcmin) with a mass deposition rate of $\dot{M} \sim 1 \mathrm{M}_{\odot}$ year $^{-1}$. The predicted spectrum is clearly different from the observed one. A way to modify the predicted spectrum in the direction of the observed one, is to assume that the central region of M87 is highly absorbed by cold or warm material to reduce the low energy part of the broad Fe line feature. The actual absorption can be determined directly, however, by using the power law spectrum of the M87 AGN with the result that there is no significant excess absorption above the known value of the galactic absorption column density of about $1.8 \times 10^{20} \mathrm{~cm}^{-2}$. A very good fit to the observed spectrum is then obtained by assuming that the bulk of the plasma in the central region of the M87 halo only occupies a narrow temperature interval of about 1.44-2 keV (Böhringer et al. 2002) as shown in the right panel of Fig. 23. 

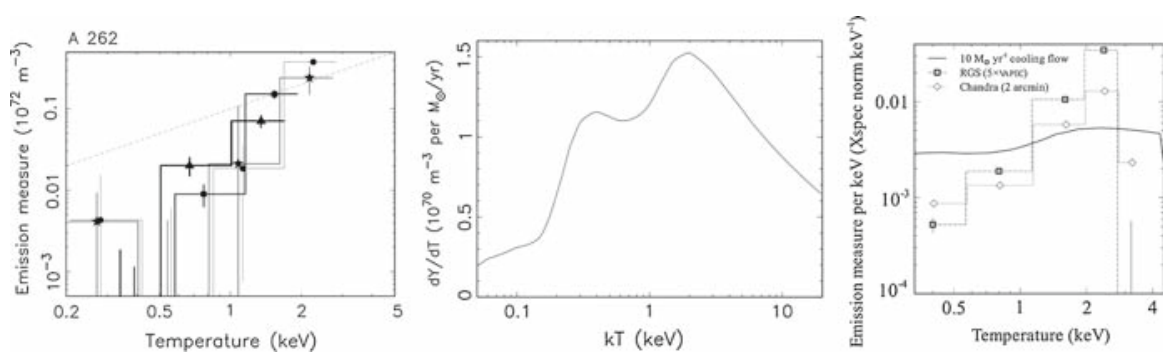

Fig. 24 Left Differential emission measure distribution as a function of temperature of the plasma in the cool core region of the cluster Abell 262 as deduced from a spectral analysis of XMM-Newton RGS observations. The results are derived for the innermost four shells: 0-0.5 arcmin (triangles), $0.5-1$ arcmin (squares), 1-2 arcmin (stars), 2-3 arcmin (circles). For comparison the dashed line shows an approximation to the slope of the isobaric cooling flow model (Kaastra et al. 2004). Middle Differential emission measure distribution for the isobaric cooling flow model, for 0.5 solar abundance (Kaastra et al. 2004) Right Differential emission measure distribution of the plasma in the inner 2 arcmin region in the core of the Centaurus cluster of galaxies. The results from a spectral analysis of XMM-Newton RGS and CHADRA are shown for comparison. The solid line shows the expected emission measure distribution for a $10 \mathrm{M}_{\odot}$ year $^{-1}$ isobaric cooling flow at solar metallicity, cooling from a temperature of 4.5-0.08 keV (Sanders et al. 2008)

It has to be noted that the interpretation of spectra from cooling flows had an interesting history already before the launch of Chandra and XMM-Newton. For example Ikebe et al. (1997) and Makishima (1999) already argued on the basis of ASCA spectroscopy with similar arguments as those given above for M87, that the prediction of cooling flow models is not met by the observations. The counterargument to keep the cooling flow model alive at the time was to postulate internal absorption (see, e.g. Allen et al. 2001). The spectral resolution and sensitivity of $A S C A$ was not quite good enough, however, to reject the cooling flow model in the very compelling way as now done with the XMM-Newton results. Even earlier, spectroscopic analysis of the X-ray halo of M87 with the Crystal Spectrometer on board of the Einstein satellite showed the emission lines for the cool phases expected in cooling flows (Canizares et al. 1979, 1982), that we are now missing in modern observations. This evidence for steady state cooling in cooling flows gave actually one of the strongest supports to the cooling flow scenario at the time. It is now clear, however, that the results obtained in these early days were most probably features of spectral noise.

Deep observations of CC regions, in particular with the XMM-Newton RGS instrument, have now also been used to characterize the multi-temperature structure in CCs in more detail in terms of the emission measure (or luminosity) distribution of the plasma as a function of temperature (e.g. Peterson et al. 2003; Kaastra et al. 2004). This works well only in colder systems. Figure 24 shows in the left panel the differential emission measure distribution in the CC region of the cluster Abell 262 in the innermost four shells out to 3 arcmin. These observations can be compared to the expected emission measure distribution for the model of an isobaric cooling flow, shown in the middle panel of Fig. 24 (Kaastra et al. 2004). While the temperature distribution is not isothermal, we clearly note that the emission measure distribution falls off much more steeply than the model expectations toward lower temperatures, highlighting again the fact that less plasma is observed at lower temperatures than required for the cooling flow model. A similar result is shown-in the right panel of 


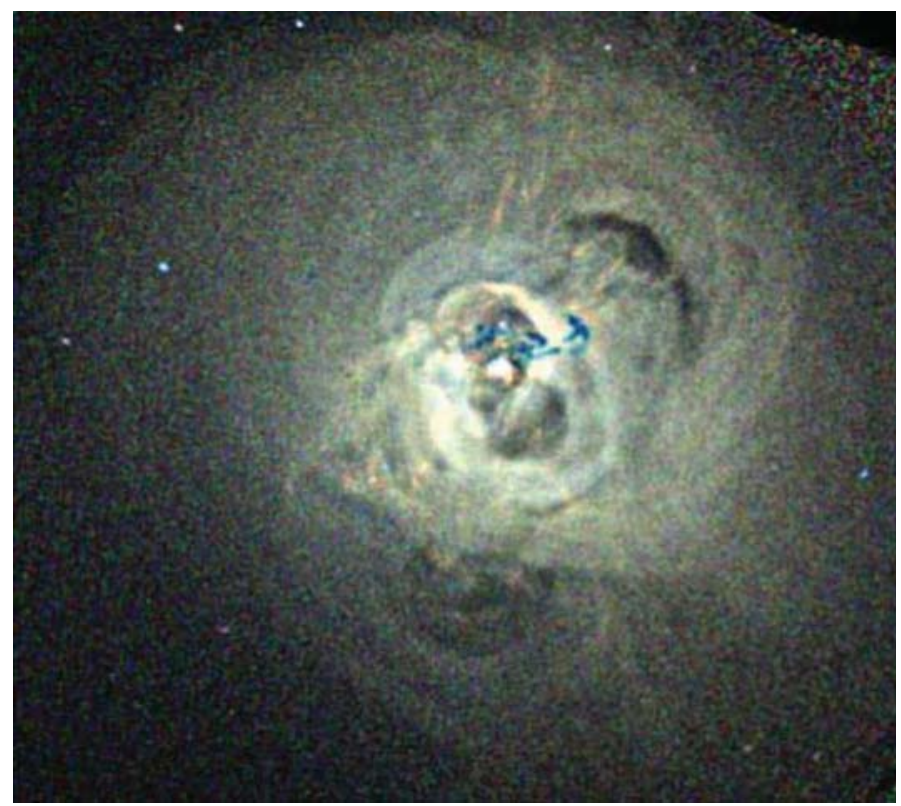

Fig. 25 False color image of the central region of the Perseus cluster produced from images in three energy bands $0.3-1.2,1.2-2$, and $2-7 \mathrm{keV}$. An image smoothed on a scale of 10 arcsec (with $80 \%$ normalization) has been subtracted from the image to highlight regions of strong density contrast. The image shows a series of nearly concentric "ripples" which are interpreted as sound waves or weak shock waves set off by the activity of the central AGN (Fabian et al. 2006)

Fig. 24-for the case of the Centaurus cluster taken from Sanders et al. (2008) based on a very deep XMM-Newton observations of the cluster with the RGS instruments as well as deep Chandra data. Here we can follow the local temperature distribution of the plasma over a full decade in temperatures from about 0.4 to $4 \mathrm{keV}$. But again the contribution of the very low temperature phases is much less than what is expected for unimpeded cooling.

As the change from the cooling flow paradigm to an AGN feedback scenario seems to be now widely accepted, the interest has shifted to the question: how is the ICM actually heated by AGN interaction? One of the most important earlier arguments in favor of cooling flows in the absence of feedback was that any possible heating mechanism has to be very well fine tuned, to exactly provide the balance to cooling. If too much heat is produced, it disperses the observed dense gas cores in cooling flows. Secondly, we observe increasing entropy profiles in the ICM of clusters (Fig. 13). It therefore has to be explained how central heating can work without inverting the entropy profiles leading to the dissipation of heat by convection. Both requirements need to be met and seem almost improbable (Fabian 1994). This makes it even more interesting now to understand how nature achieves this fine tuning.

One of the first, best studied cases is that of the Perseus cluster, which has now been observed with the Chandra observatory for more than 1 Million seconds as proposed by Fabian et al. (2005) providing the most detailed picture of a central CC region. Figure 25 shows a multi-color image of the central region of the Perseus cluster from 


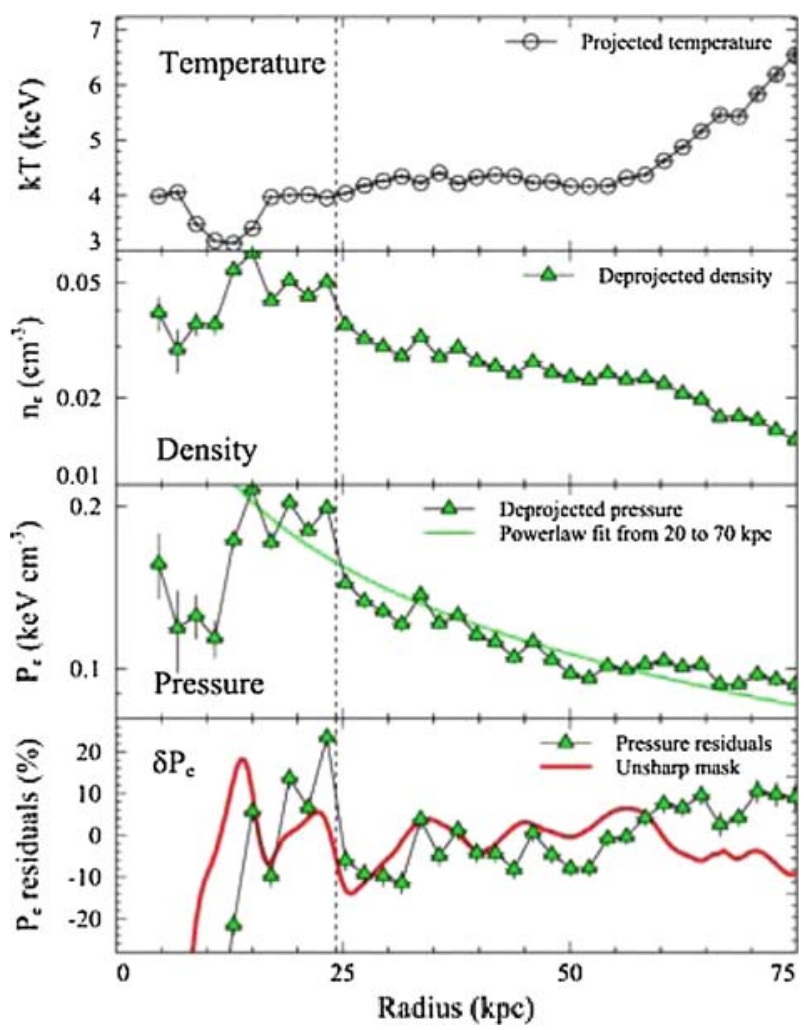

Fig. 26 Temperature, density, pressure, and pressure residual profiles in the core region ICM of the Perseus cluster in a sector to the North-East (Fabian et al. 2006). The red line shows the ripples from an unsharp mask image. The dashed line marks the position of a shock front

Fabian et al. (2005). The "false color" image was produced from images in three energy bands $0.3-1.2,1.2-2$, and $2-7 \mathrm{keV}$. An image smoothed on a scale of 10 arcsec (with $80 \%$ normalization) has been subtracted from the image to highlight regions of strong density contrast. The central, bright part of the image shows two features of low surface brightness, interpreted as two cavities of the X-ray emitting plasma filled with the relativistic plasma from the radio jets of the central AGN. This region is surrounded by a series of nearly concentric "ripples" which are more clearly brought out by the unsharp masking processing of the image.

A more detailed analysis of the density and temperature variations across the ripples, shown in Fig. 26, implies typical pressure variations associated with the ripples with amplitudes of about $\pm 5-10 \%$, which are interpreted as sound waves or very weak shock waves in the innermost region. As detailed in Fabian et al. (2005) the dissipation of the sound waves - if the viscosity is sufficiently high — can balance the radiative cooling in the $\mathrm{CC}$ by heating with the realistic assumption that the sound waves cross the $\mathrm{CC}$ region in about $1 / 50$ of the cooling time.

Fabian et al. (2006) have also carried out multi-temperature plasma modeling in various regions of the cluster center and produced mass distribution maps of plasma 


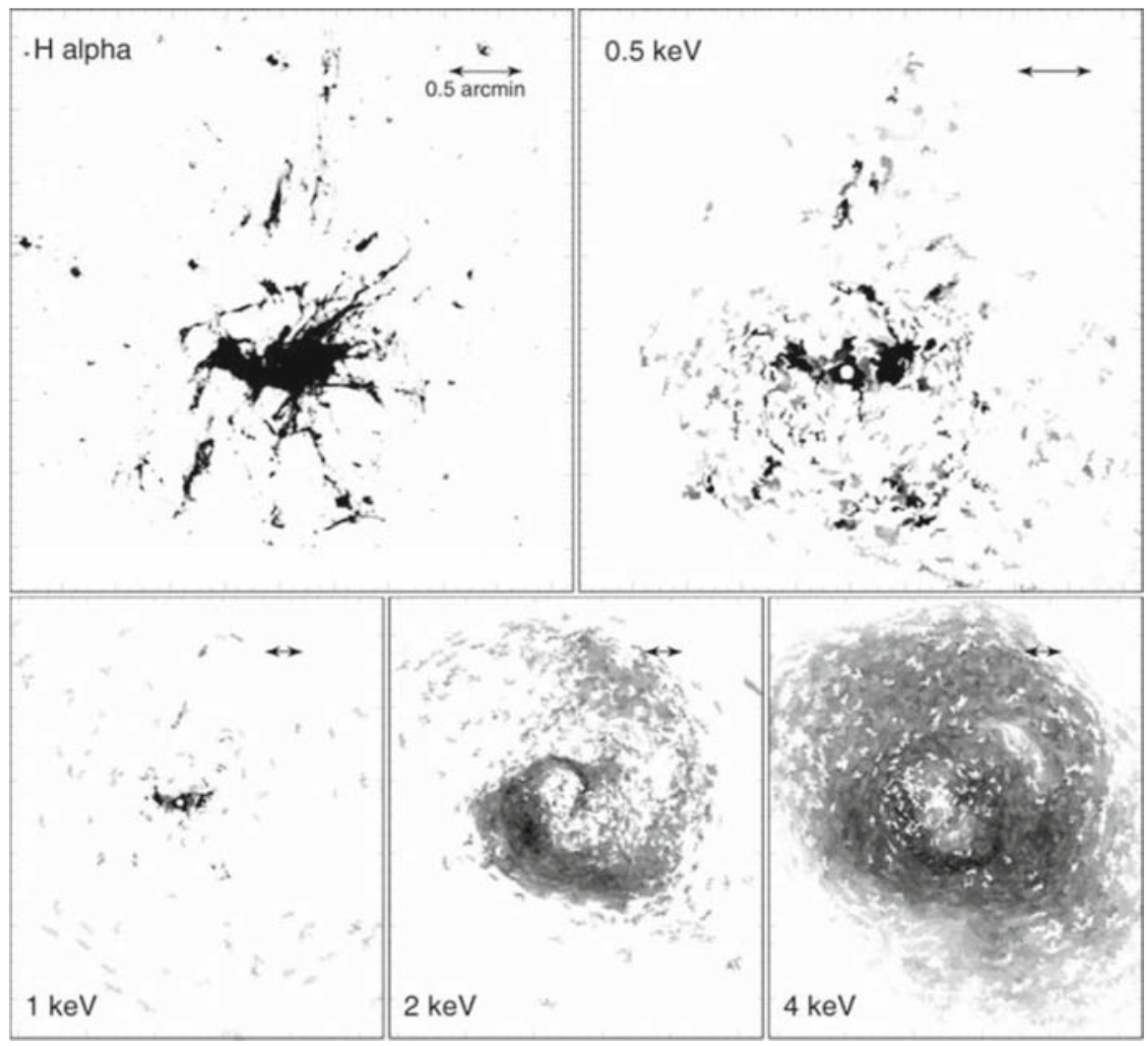

Fig. $27 \mathrm{H} \alpha$ image in the halo of NGC 1275 in the center of the Perseus cluster (from Conselice et al. 2001, upper left) shown on the same scale for comparison with maps of the mass distribution of different temperature components, at $0.5,1,2$, and $4 \mathrm{keV}$, of the ICM in the center of the Perseus cluster (Fabian et al. 2006)

at temperatures around $0.5,1,2$ and $4 \mathrm{keV}$. These maps are shown in comparison to a map of the optical $\mathrm{H} \alpha$ emission (observed by Conselice et al. 2001) in Fig. 27. The distribution of the lowest temperature phase around $0.5 \mathrm{keV}$ has a striking similarity to the $\mathrm{H} \alpha$ map. There is relatively little plasma at $1 \mathrm{keV}$, mostly in the very center of the CC. The $2 \mathrm{keV}$ phase coincides mostly with the denser regions of the ICM. This detailed picture of the central region of the Perseus cluster given in a series of papers by Fabian et al. and Sanders et al. well illustrate the frontier of the application of X-ray spectroscopy and thermodynamic analysis of the ICM in the bright central regions of clusters.

To show that the physics of the $\mathrm{CC}$ regions seems to be quite diverse and that we can obviously not easily generalize the behavior of the Perseus cluster to all CC clusters we show two further examples of systems studied in detail, that of M87 and of Hydra A. M87 was studied in very much detail by a combination of a 500 ks exposure with Chandra (Forman et al. 2005, 2007) and a $120 \mathrm{ks}$ exposure with XMM-Newton (Simionescu et al. 2007, 2008). The X-ray halo of M87 is the closest of all CC regions 

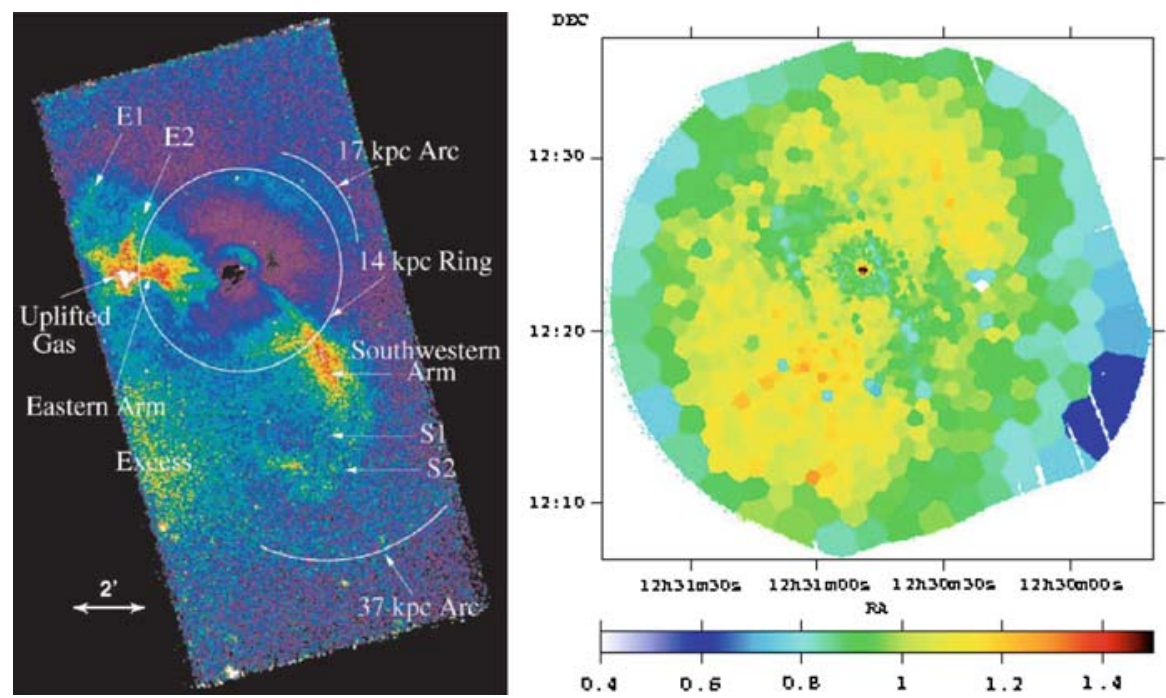

Fig. 28 Left: Chandra image of the X-ray halo of M87 in the 0.5-2.5 keV band (Forman et al. 2005). Several features are marked on this image: the surface brightness enhancements coinciding with the radio lobes (SW arm and E arm) and several sharp surface brightness discontinuities (14 kpc ring, $17 \mathrm{kpc}$ arc, and $37 \mathrm{kpc}$ arc) which are identified as shock fronts. Right Map of the pressure deviations from a smooth spherical symmetric model derived from a deep XMM-Newton observation of the M87 halo (Simionescu et al. 2007). The $14 \mathrm{kpc}$ ring is now clearly revealed as a pressure discontinuity supporting the shock interpretation. The regions of the radio lobes (SW arm and E arm) are now showing up as zones of pressure deficit, which is probably compensated for by the unseen pressure of the relativistic radio plasma

in galaxy clusters and can therefore be studied at the highest spatial resolution. Also the AGN in the center of M87 is active and is interacting with the ambient ICM through relativistic jets (e.g. Böhringer et al. 1995; Churazov et al. 2001). The deep Chandra exposure shows a surface brightness discontinuity which can be almost followed over the entire circumference of a ring around the nucleus with a radius of $14 \mathrm{kpc}$ (Fig. 28, Forman et al. 2005, 2007). Another arc-shaped discontinuity is seen over a smaller sector region at the radius of $17 \mathrm{kpc}$. These features are identified as shock waves in Forman et al. (2005, 2007). A detailed spectroscopic analysis of the M87 X-ray halo with XMM-Newton can corroborate this interpretation and give enhanced physical constraints. While the density discontinuity seen with Chandra implies a shock Mach number of about 1.2, the temperature jump seen in the projected spectra is about $5 \%$ at about $2 \mathrm{keV}$ and implies a shock Mach number of $>1.05$ without deprojection correction (Simionescu et al. 2007). Thus the temperature enhancement identifies the surface brightness discontinuity definitely as a weak shock front. The shock front has also been visualized in a pressure map of the M87 halo constructed from the XMMNewton data shown in Fig. 28 (Simionescu et al. 2007). Similar to the hydrodynamic maps described above this map has been derived from spectral modeling in many spatial pixel, which in this case have been constructed by means of a Voronoi tessellation technique developed by Diehl and Statler (2006) and originally by Cappellari and Copin (2003) such that each pixel has a spectral signal to noise of at least 100. To enhance the visibility of discontinuities in the presence of the strong pressure gradient, 

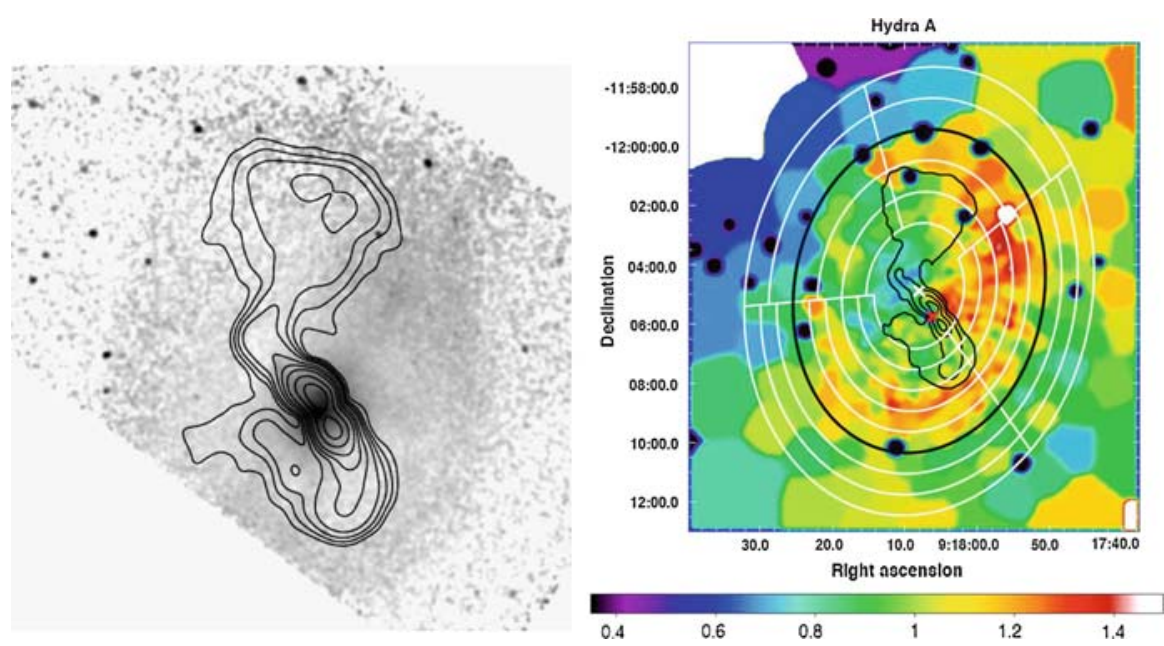

Fig. 29 Left: Chandra image of Hydra A with the radio lobes superposed (Nulsen et al. 2005). An oval shaped surface brightness discontinuity at a radius of about $200-300 \mathrm{kpc}$ can be noted that is identified with a shock wave caused by an energetic radio jet outburst. Right Map of the pressure deviations from a smooth azimuthally symmetric model derived from a deep XMM-Newton observation of Hydra A (Simionescu et al. 2009a). The location of the X-ray surface brightness discontinuity observed in the Chandra image is approximated by the elliptical black line. A clear pressure jump can be seen almost all along this line indicating a shock front with a Mach number of about 1.3

a smooth, spherically symmetric model has been subtracted from the pressure distribution shown in Fig. 28. The map clearly shows a pressure enhancement at the shock radius of $3^{\prime}(14 \mathrm{kpc})$ which implies without projection correction a Mach number larger than 1.08. There are some further interesting features seen in the pressure map. Outside the shock front we note two regions of lower pressure in the NE and in the SW. These are the regions where radio lobes filled with relativistic plasma emerging from the central AGN are observed. This observation thus implies that (at least part of) the missing pressure in these regions comes from the pressure of the relativistic plasma in the lobes, which does not contribute to the X-ray emission analyzed here. Further insight into the physics of this interesting $\mathrm{CC}$ region in M87 will be described in connection with the analysis of the element abundance distribution discussed in the next section.

In a combined Chandra and XMM-Newton study of the central region of the galaxy cluster associated with the radio source Hydra A, a similar front diagnostics has also been performed by Nulsen et al. (2005) and Simionescu et al. (2009a). Here the shock feature has a radial extent of $200-300 \mathrm{kpc}$ and hints at an event which was much more powerful by about two orders of magnitude than that in M87. Figure 29 shows again the surface brightness discontinuity clearly detected in the high angular resolution image obtained with the Chandra observatory (Nulsen et al. 2005). A deep XMMNewton observation then provides higher photon statistics that allows us to study this discontinuity spectroscopically. Firstly, we can again visualize the shock front as a pressure jump in the XMM-Newton based pressure map shown in Fig. 29 (Simionescu et al. 2009a). A more detailed analysis of the temperature and density distribution 

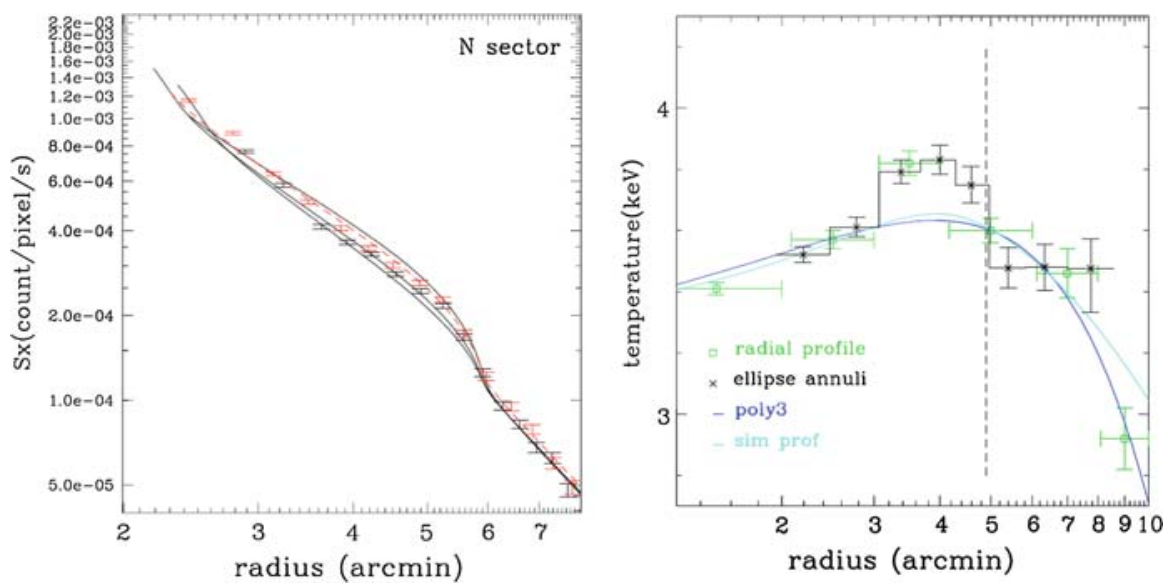

Fig. 30 Left Model of the effect of a shock front with different Mach numbers predicting the observed surface brightness distribution in the XMM-Newton image of Hydra A (Simionescu et al. 2009a). Right Temperature profile around the shock front compared to a smooth model (blue lines). The observed temperature and density (surface brightness) jumps are consistent with a shock wave with Mach number $\sim 1.3$ (Simionescu et al. 2009a)

around the shock front in several sectors, shown in Fig. 30, provides more detailed information about the shock strength. Modeling the discontinuity in three dimensions and fitting the projected model to the data implies a Mach number of the best fitting model of about 1.2-1.3 (Simionescu et al. 2009a). Simple one-dimensional modeling of the evolution of the shock driven by the energy input of the radio lobes as well as detailed three-dimensional hydrodynamical simulations provide good estimates for the age and the total energy of the event causing the shock wave, with values for the age of $\sim 2 \times 10^{7}$ years and the energy of $10^{61} \mathrm{erg}$ (Nulsen et al. 2005; Simionescu et al. 2009a).

These few examples show the complex and probably very diverse physics prevailing in cluster $\mathrm{CC}$ regions. We hope that further detailed studies of a larger sample of $\mathrm{CC}$ regions will help to generalize the scenario of CC heating. A major break through is also expected here from future high resolution spectroscopy, which will allow us to observe turbulent and bulk motions in the ICM as described in Sect. 7.

\section{What chemical abundance measurements tell us}

Clusters of galaxies are unique laboratories for the study of the nucleosynthesis and chemical enrichment of the Universe. Their deep gravitational potential wells keep all the metals produced by the stellar populations of the member galaxies within the cluster. The dominant fraction of these metals reside within the hot ICM. The chemical abundances measured in the intra-cluster plasma thus provide us with a "fossil" record of the integral yield of all the different stars (releasing metals in supernova explosions and winds) that have left their specific abundance patterns in the gas prior and during cluster evolution. 

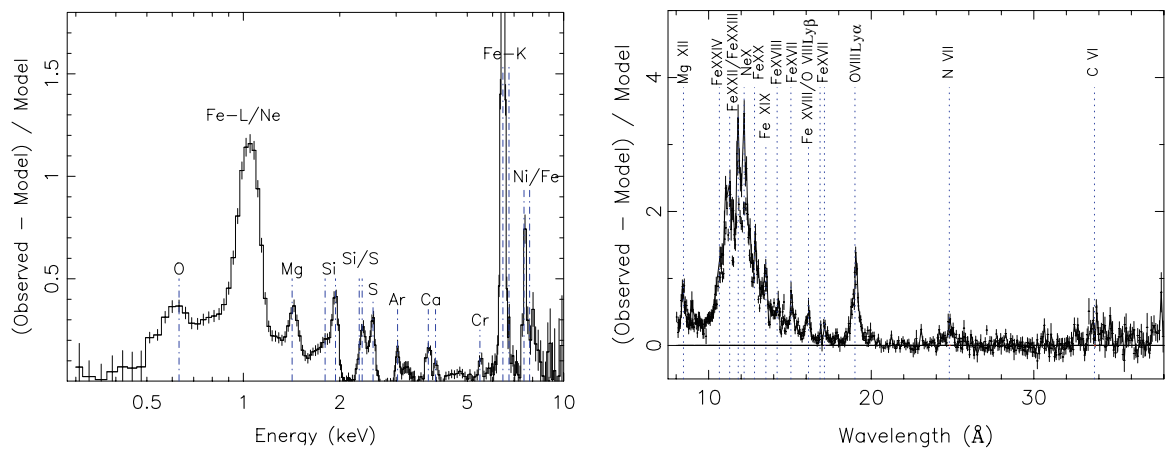

Fig. 31 Left panel The line spectrum of the cluster 2A 0335+096, as observed with XMM-Newton EPIC (from Werner et al. 2006b). Right panel Line spectrum of M 87, as observed with XMM-Newton RGS (from Werner et al. 2006a). This representation of the spectrum has been obtained by recalculating the spectrum with the best fit values without lines, and using the result to subtract it from the observed spectrum as indicated on the $Y$-axis. While the CCD spectra are plotted as a function of the observed energy, the grating spectra are shown as a function of the observed wavelength

X-ray spectroscopy provides an accurate measure of metal abundances in the ICM. These abundances put constrains on nucleosynthesis and on the star formation history of the clusters, and as long as the stellar populations where the cluster metals were synthesized are representative, these measurements allow us to put constraints on the chemical evolution of the Universe as a whole.

The CCD type detectors on Chandra, XMM-Newton, and Suzaku allow us to detect the emission lines of $\mathrm{O}, \mathrm{Ne}, \mathrm{Mg}, \mathrm{Si}, \mathrm{S}, \mathrm{Ar}, \mathrm{Ca}, \mathrm{Fe}$, and $\mathrm{Ni}$ (see the left panel of Fig. 31). The $2 \mathrm{p}-1 \mathrm{~s}$ Ne lines at $1.02 \mathrm{keV}$ are in the middle of the Fe-L complex (lying between 0.8 and $1.4 \mathrm{keV}$ ). The energy resolution of the CCD type detectors is not sufficient to resolve the individual lines in the Fe-L complex, which makes the Ne abundance determination very sensitive to uncertainties in the temperature modeling. However, the abundance of $\mathrm{Ne}$ in the cores of cooling core clusters, observed with $X M M$ Newton with sufficiently deep exposures, can be well determined using the high spectral resolution of the Reflection Grating Spectrometers (RGS). The RGS has a higher spectral resolution than the CCDs. It resolves the Fe-L complex into individual lines and allows for accurate measurements of the equivalent widths of $\mathrm{O}, \mathrm{Ne}, \mathrm{Mg}$, and Fe. In the case of deep observations of nearby bright, relatively cool clusters and elliptical galaxies even the spectral lines of $\mathrm{C}$ and $\mathrm{N}$ can be detected (see the right panel of Fig. 31). The high resolution transmission grating spectrometers on Chandra are because of their small effective area unfortunately not well suited for cluster spectroscopy.

The equivalent widths of the observed lines are under the assumption of collisional equilibrium directly converted into abundances of the corresponding elements. Because the ICM is a relatively uncomplicated physical environment (ionization nonequilibrium or optical depth effects are minimal) these abundances are relatively robust.

Abundances are often shown in the literature with respect to the outdated Solar abundances by Anders and Grevesse (1989) or with respect to newer sets of Solar and proto-solar abundances by Lodders (2003). The more recent Solar abundance 
determinations of $\mathrm{O}, \mathrm{Ne}$, and Fe by Lodders (2003) are 30\% lower than those given by Anders and Grevesse (1989). The Solar abundances of O and Ne reported by Grevesse and Sauval (1998) are higher and the abundance of Fe is slightly lower than those reported by Lodders (2003).

\subsection{Early pioneering work on chemical abundances}

The first evidence that the ICM is strongly polluted by metals ejected from stars in the cluster galaxies came with the discovery of the Fe-K line emission in the spectrum of the Perseus, Coma, and Virgo clusters by the Ariel $V$ and $O S O-8$ satellites (Mitchell et al. 1976; Serlemitsos et al. 1977). Spectral analysis of samples of clusters observed with $O S O-8$ and HEAO-1 A2 showed that the ICM is enriched by Fe to $0.3-0.5$ of the Solar abundance value (Mushotzky et al. 1978; Mushotzky 1984).

Until the launch of ASCA in 1993, only the Fe abundance was accurately measured in many clusters. ASCA for the first time detected emission features from many different elements $(\mathrm{O}, \mathrm{Ne}, \mathrm{Mg}, \mathrm{Si}, \mathrm{S}, \mathrm{Ar}, \mathrm{Ca}, \mathrm{Fe}$, and $\mathrm{Ni}$ ) in the ICM, opening thus a new chapter in the chemical enrichment studies. While core collapse supernovae $\left(\mathrm{SN}_{\mathrm{CC}}\right)$ produce large amounts of $\mathrm{O}, \mathrm{Ne}$, and $\mathrm{Mg}$, Type Ia supernovae (SN Ia) produce large quantities of $\mathrm{Fe}, \mathrm{Ni}$, and $\mathrm{Si}$-group elements $(\mathrm{Si}, \mathrm{S}, \mathrm{Ar}$, and $\mathrm{Ca}$ ), but only very little $\mathrm{O}, \mathrm{Ne}$, and $\mathrm{Mg}$. Early ASCA measurements of $\mathrm{O}, \mathrm{Ne}, \mathrm{Si}, \mathrm{S}$, and $\mathrm{Fe}$ abundances in four clusters suggested that the ICM enrichment is dominated by $\mathrm{SN}_{\mathrm{CC}}$ (Mushotzky et al. 1996). However, few years later analyzing ASCA data, Finoguenov et al. (2000) showed that while the $\mathrm{SN}_{\mathrm{CC}}$ products are uniformly distributed in the ICM, the chemical enrichment of the cluster cores is dominated by SN Ia. An interesting result based on ASCA by Fukazawa et al. (1998) and Baumgartner et al. (2005) showed that the Si abundance and the $\mathrm{Si} / \mathrm{Fe}$ ratio increase from the poorer to the richer clusters, suggesting that the relative contribution of $\mathrm{SN}_{\mathrm{CC}}$ increases toward the more massive clusters. In a pioneering work Dupke and White (2000) used the abundances measured in three clusters of galaxies to put constraints on SN Ia models. They used the $\mathrm{Ni} / \mathrm{Fe}$ ratio to discriminate between different SN Ia explosion scenarios available in the literature. Their work preferred the W7 "deflagration" models over the "delayed detonation" models.

The ASCA data of the Centaurus cluster were the first to show a strong Fe abundance peak at the cooling core (Fukazawa et al. 1994). Allen and Fabian (1998) used ASCA spectra and ROSAT images to show a segregation between the metallicities of clusters with and without cooling cores and suggested that it is caused by the presence of metallicity gradients coupled with the sharply peaked surface brightness profiles of the cooling core clusters. Unfortunately, the large point-spread function of ASCA did not allow to investigate the spatial abundance distributions in detail. Using the BeppoSAX satellite, which had a better spatial resolution than ASCA, De Grandi and Molendi (2001) clearly showed that while non-cooling core clusters have flat Fe abundance profiles, the metallicity distribution of cooling core clusters has a gradient with a central peak (see Fig. 32). Abundance gradients were also found in 9 out of 10 galaxies and groups of galaxies by Buote (2000a), who analyzed ROSAT data of these $\sim 1 \mathrm{keV}$ systems. 
Fig. 32 The average radial distribution of the Fe abundance for a sample of cooling core (filled circles) and non-cooling core (empty circles) clusters observed with BeppoSAX (from De Grandi et al. 2004)

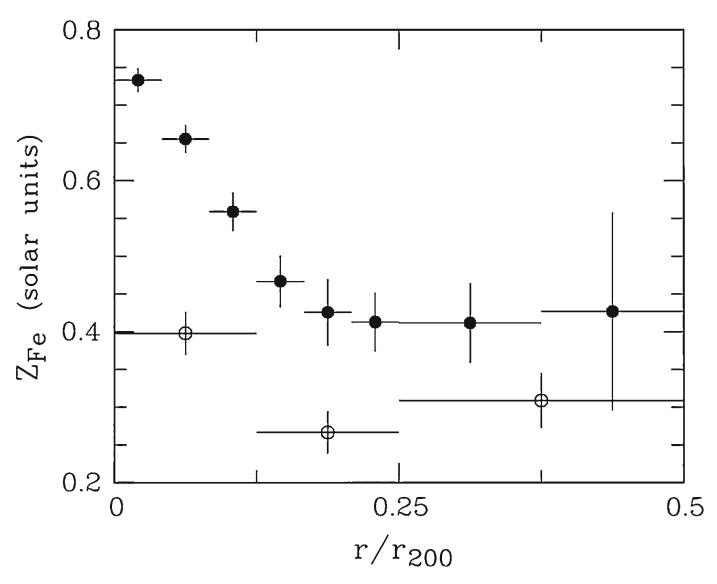

$A S C A$ data were furthermore used for a pioneering search for evolution in cluster metallicities, revealing a lack of evolution out to redshift $z \sim 0.4$ (Mushotzky and Loewenstein 1997; Rizza et al. 1998) and finding no evidence for a decrease at higher redshifts (Donahue et al. 1999).

\subsection{Chemical enrichment in cooling core clusters}

The emerging picture of the chemical enrichment in clusters of galaxies at the time of the launch of XMM-Newton and Chandra was that of an early enrichment of the ICM by $\mathrm{SN}_{\mathrm{CC}}$, the products of which are today well mixed and homogeneously distributed, and a subsequent more centrally peaked enrichment by SN Ia which continue to explode in the $\mathrm{cD}$ galaxy for a long time after the cluster is formed. According to this picture the observed peaked distribution of the $\mathrm{Fe}$ abundance is largely due to the SN Ia in the cD galaxy.

The first abundance studies performed with XMM-Newton seemed to confirm this scenario. The radial abundance profiles of the cooling core cluster Abell 496 showed a flat distribution of $\mathrm{O}, \mathrm{Ne}, \mathrm{Mg}$, which are predominantly produced by $\mathrm{SN}_{\mathrm{CC}}$, but a centrally peaked distribution of $\mathrm{Si}, \mathrm{S}, \mathrm{Ar}, \mathrm{Fe}$, and Ni (Tamura et al. 2001). However, while most of the Fe and $\mathrm{Ni}$ is produced by $\mathrm{SN} \mathrm{Ia}, \mathrm{Si}, \mathrm{S}, \mathrm{Ar}$, and $\mathrm{Ca}$ (so called $\alpha$ elements) are produced by both supernova types in similar proportions. In many systems, Si has a relatively well determined abundance value and in a cluster with a flat $\mathrm{O}$ profile one would expect to see a shallower Si abundance gradient than that of Fe.

However, this has never been observed. In the most nearby cluster cooling core, M87, the Si abundance has a similar gradient as that of Fe, but at the same time it has a low $\mathrm{O} / \mathrm{Fe}$ ratio in the core that increases toward the outer regions (Böhringer et al. 2001; Finoguenov et al. 2002). A similar abundance pattern was observed in the cluster sample analyzed by Tamura et al. (2004), in the Perseus cluster (Sanders et al. 2004), in Abell 85 (Durret et al. 2005), in the Centaurus cluster (Matsushita et al. 2007a; Sanders and Fabian 2006), in the group NGC 5044 (Buote et al. 2003), in Abell 1060 (Sato et al. 2007), and in AWM7 (Sato et al. 2008). 
To solve this discrepancy in M 87, Finoguenov et al. (2002) proposed that there are two types of SN Ia with different $\mathrm{Si} / \mathrm{Fe}$ yields. Those with longer delay times and higher Si yields are today dominating the enrichment of elliptical galaxies, such as the $\mathrm{cD}$ galaxies in the centers of cooling core clusters, and those with shorter delay times and lower Si yields dominate the enrichment in the ICM at larger radii, because the bulk of them exploded in the time of the strongest star formation period (for more details on SN Ia models see Sect. 5.3).

Recently, Simionescu et al. (2009b) determined radial abundance profiles for O, Si, $\mathrm{S}$, and $\mathrm{Fe}$ in the Hydra A cluster. They found that all elements have a centrally peaked distribution. In order to investigate the radial abundance distributions in clusters in more detail, they combined their profiles with those determined for 5 other clusters with high quality X-ray data (M87, Centaurus, Fornax, 2A 0335+096, Sérsic 159-03, and Abell 1060). In the combined data set they find that the $\mathrm{O}$ distribution also peaks in the cluster core and decreases with radius as $d \mathrm{O} / d\left(\log r / r_{200}\right)=-0.48 \pm 0.07$. But the Fe abundance still decreases faster: $d \mathrm{Fe} / d\left(\log r / r_{200}\right)=-0.72 \pm 0.04$, and therefore the fraction of $\mathrm{O}$ with respect to $\mathrm{Fe}$ increases with radius as $d(\mathrm{O} / \mathrm{Fe}) / d\left(\log r / r_{200}\right)=$ $0.25 \pm 0.09$. The $\mathrm{Si}$ abundance profile, however, remains exactly the same as that of $\mathrm{Fe}$, it decreases with radius as: $d \mathrm{Si} / d\left(\log r / r_{200}\right)=-0.72 \pm 0.04$.

Surprisingly, for the group NGC 507, Sato et al. (2009) found steep abundance gradients peaking on the cluster core not only for $\mathrm{Si}, \mathrm{S}$, and Fe but also for $\mathrm{Mg}$. They also found a mildly centrally peaked abundance distribution for O. The Suzaku satellite has a better sensitivity in the $\mathrm{O}$ line energy and it allows for better measurements of the $\mathrm{Mg}$ lines in the low surface brightness cluster outskirts than XMM-Newton, which has strong instrumental lines and effective area calibration problems at the $\mathrm{Mg}$ energy. As shown in Fig. 33 the $\mathrm{Mg} / \mathrm{Fe}$ ratio as measured with Suzaku is consistent with being flat out to $0.2 r_{180}$ in Fornax (Matsushita et al. 2007a), HCG 62 (Tokoi et al. 2008), and in NGC 507 (Sato et al. 2009). Since both $\mathrm{Mg}$ and $\mathrm{O}$ are produced by $\mathrm{SN}_{\mathrm{CC}}$ their radial profiles should follow each other. However, even though the error-bars are large, the $\mathrm{O} / \mathrm{Fe}$ ratio seems to increase with the radius in these three systems. The different abundance profiles for $\mathrm{Mg}$ and $\mathrm{O}$ might be due to systematic uncertainties in regions with low surface brightness where the confusion with the strong O VIII Galactic foreground line becomes an issue. The most straightforward interpretation of these profiles is that the $\mathrm{SN}_{\mathrm{CC}}$ products are also centrally peaked, as indicated by $\mathrm{Mg}$, and the measured peak in the $\mathrm{O}$ abundance profile is shallower because of the confusion with the Galactic $\mathrm{O}$ VIII foreground lines which become important in the faint cluster outskirts.

Based on the Fe abundance profiles, De Grandi et al. (2004) and Böhringer et al. (2004) concluded that the central abundance peak is due to the metals released by the stellar population of the $\mathrm{cD}$ galaxy. To produce the observed abundance peaks requires long ( $\gtrsim 5$ Gyr) enrichment times (Böhringer et al. 2004). In the absence of mixing the metallicity profiles should follow the optical light profiles of the $\mathrm{cD}$ galaxies. But the observed metal mass profiles are much less peaked than the optical light, which implies that the metals get mixed and transported out to larger radii, most probably by the AGN/ICM interaction (e.g. Rebusco et al. 2005, 2006).

The role of the AGN in the metal transport was both theoretically and observationally best investigated in M 87. Churazov et al. (2001) proposed a scenario where buoyant bubbles of radio emitting relativistic plasma, produced by the jets of the AGN, 


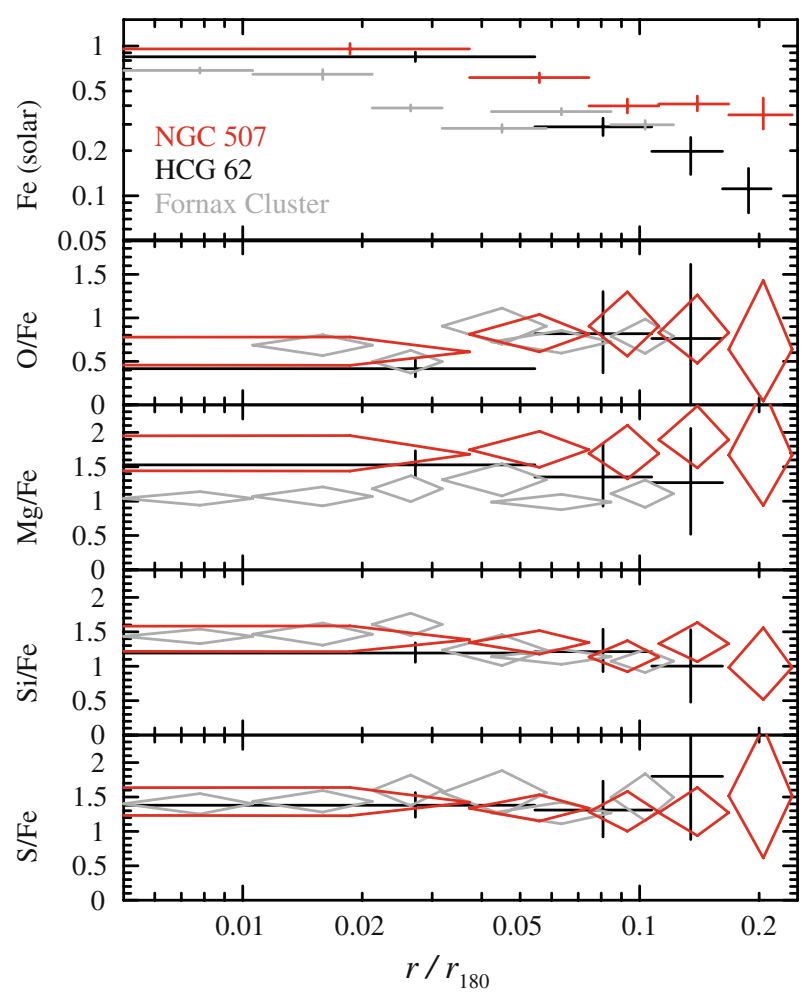

Fig. 33 Radial profile of abundance ratios for NGC 507 (Sato et al. 2009), HCG 62 (Tokoi et al. 2008), and the Fornax cluster (Matsushita et al. 2007a). All the abundance ratios are consistent with having a flat radial profile, except $\mathrm{O} / \mathrm{Fe}$ which shows an indication for an increase with the radius. The abundance ratios are in the solar units of Anders and Grevesse (1989)

uplift the cool metal rich gas from the central parts of the $\mathrm{cD}$ galaxy. This scenario was confirmed by observations of cool metal rich gas, distributed along the radio lobes (see Fig. 34, Simionescu et al. 2008). The Fe abundance of the uplifted gas (a mixture of ICM, stellar mass loss, and SN Ia products) is $\sim 2.2$ times solar and its mass is $\approx 5 \times 10^{8} M_{\odot}$. It takes $\approx 30-110 \mathrm{Myr}$ to produce the metals in the cool gas. The relative abundances of the uplifted gas are consistent with those of the ambient ICM, indicating that the dominant fraction of metals in the cluster core was uplifted after the last major epoch of star formation and the relative enrichment rate by SN Ia with respect to that by stellar winds remains fairly constant with time. Uplift of metal rich cool gas from the $\mathrm{cD}$ galaxy by buoyantly rising bubbles of radio emitting plasma was also observed in the Hydra A cluster of galaxies (Simionescu et al. 2009b). The total mass of the uplifted Fe in Hydra A is $1.7 \times 10^{7} M_{\odot}$.

Can the abundance peaks be produced by only stellar winds and SN Ia in the cD galaxy? To explain the centrally peaked distribution of $\mathrm{O}, \mathrm{Si}$, and $\mathrm{S}$ in the Hydra A cluster would require the amount of metals produced by stellar winds to be 3-8 times higher than predicted by models or the metals produced by $\mathrm{SN}_{\mathrm{CC}}$ in the proto-cluster phase not to be mixed completely (Simionescu et al. 2009b). Centrally peaked distribution of all elements can also be produced by ram-pressure stripping of cluster galaxies (e.g. 

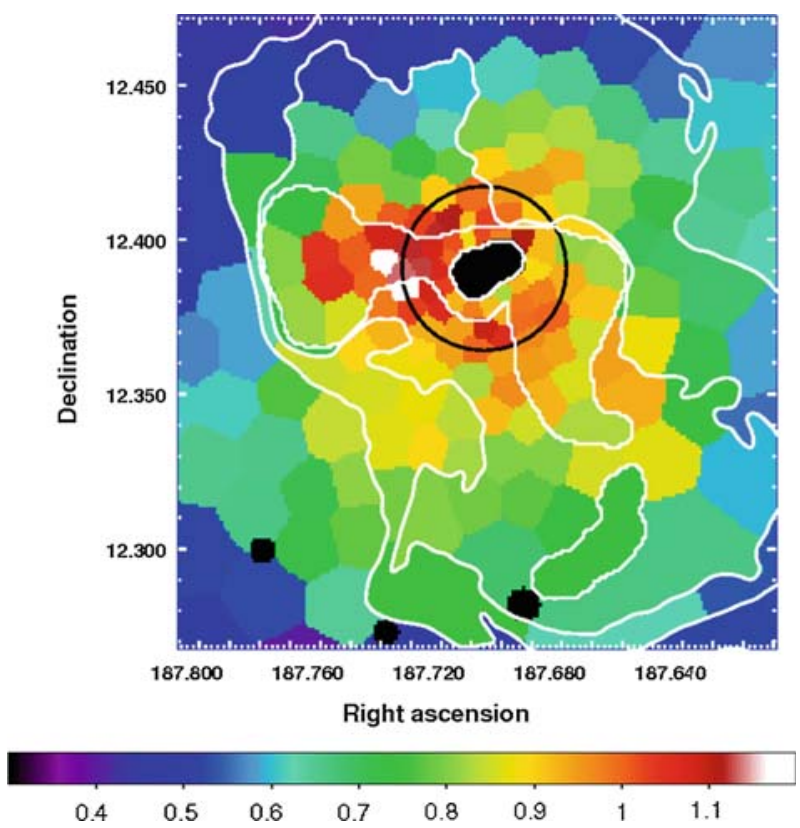

Fig. 34 Map of the Fe abundance in M 87 in Solar units indicated under the color bar. Contours of the $90 \mathrm{~cm}$ radio emission (Owen et al. 2000) are overplotted. Beyond the expected radial gradient, one clearly sees the enhanced Fe abundance in the radio arms, especially within the Eastern arm (from Simionescu et al. 2008)

Domainko et al. 2006). But both simulations and observations indicate that galaxies are getting stripped already at large distances from cluster cores and this mechanism probably does not contribute significantly to the observed abundance peaks on the relatively small spatial scales around the $\mathrm{cD}$ galaxies.

The radial metallicity gradients in cooling flow clusters often display an inversion in the center (e.g. Sanders and Fabian 2002). While an apparent drop in metal abundances can be the result of an oversimplified model in the spectral analysis of the very center of a cooling core (e.g. Buote and Fabian 1998; Molendi and Gastaldello 2001; Werner et al. 2006b; Matsushita et al. 2007a, for more discussion about spectral fitting biases see Sect. 5.5.1) in some cases this inversion seems robust. In particular the metallicity in the Perseus cluster drops very clearly at radii smaller than $40 \mathrm{kpc}$ and the central dip does not disappear when multi-temperature models and extra power-law components are introduced in the spectral fitting, or when projection effects and possible effects of resonant scattering are taken into account (Sanders et al. 2004; Sanders and Fabian 2006, 2007). The metallicity dips in the cluster cores may be related to the AGN/ICM feedback.

\subsection{Constraining supernova models using clusters}

As we mentioned before, clusters of galaxies retain all the metals produced by their stellar populations within their gravitational potential. The dominant fraction of these 
metals resides within the hot ICM (Finoguenov et al. 2003). All the elements from oxygen up to the iron group are primarily produced by supernovae. Their abundances within the ICM thus provide us with the integral yield of all the supernova explosions in the cluster.

Since the launch of the ASCA satellite there have been efforts to use the abundance patterns in the ICM to put constraints on the theoretical supernova models. These efforts were mainly focused on constraining the Type Ia explosion mechanisms. Dupke and White (2000) and Dupke and Arnaud (2001) used the Ni/Fe ratio measured in clusters by ASCA to distinguish between the SN Ia models assuming slow deflagration and delayed detonation. While the deflagration explosion mechanism of SN Ia (represented by the W7 and W70 models in the literature, Iwamoto et al. 1999) predicts high Ni/Fe ratio of 2.18-3.22 in the Solar units of Grevesse and Sauval (1998), the delayed-detonation explosion scenarios predict significantly lower $\mathrm{Ni} / \mathrm{Fe}$ ratios of 0.9-1.4 Solar. They are represented by the WDD1, WDD2, WDD3, CDD1, and CDD2 models in the literature, where the last digit indicates the density at which the flame velocity becomes supersonic (deflagration-to-detonation transition density) in units of $10^{7} \mathrm{~g} \mathrm{~cm}^{-3}$; the " $\mathrm{C}$ " and "W" refer to two different central densities $\left(1.37 \times 10^{9}\right.$ and $2.12 \times 10^{9} \mathrm{~g} \mathrm{~cm}^{-3}$, respectively) in the model at the onset of the thermonuclear runaway (Iwamoto et al. 1999). ASCA measured relatively high $\mathrm{Ni} / \mathrm{Fe}$ ratios which clearly favored the W7 SN Ia model (Dupke and White 2000; Dupke and Arnaud 2001 see also Sect. 5.5.1).

The results based on the abundances measured by XMM-Newton, Chandra, and Suzaku are not so clear and depending on the relative abundances in the given cluster sometimes deflagration models and sometimes delayed detonation models are favored.

An excellent example of the complicated situation is the Virgo cluster with M87 in its cooling core. The abundance patterns in M 87 cannot be described by any combination of $\mathrm{SN}_{\mathrm{CC}}$ and deflagration $\mathrm{SN}$ Ia models. The low Ni/Fe ratio and very high $\mathrm{Si} / \mathrm{Fe}$ ratios clearly favor delayed detonation models. The high SN Ia yield of Si-group elements in M87 imply that the Si burning in SN Ia is incomplete, as predicted by the delayed-detonation models with lower density of deflagration to denotation transition (WDD1 model). The abundance patterns in the outer region of M 87 are on the other hand characteristic of deflagration supernova models or delayed detonation models with the highest deflagration to detonation density (WDD3). Finoguenov et al. (2002) conclude that these abundance patterns confirm the diversity of SN Ia. Today, this conclusion seems to be supported by both optical supernova surveys and more recent measurements of cluster abundances.

Optical surveys show a correlation between SN Ia properties and the morphology of their host galaxy. While a population of brighter SN Ia with a slow luminosity decline is more common in late-type spiral and irregular galaxies with recent star formation (indicating a short delay time between their formation and the explosion), a fainter and more rapidly decaying population of SN Ia is more common in early-type galaxies (Hamuy et al. 1996; Ivanov et al. 2000). This is clearly seen in Fig. 35 from Gallagher et al. (2005) which shows the morphological type of the parent galaxy versus the decline rate of SNe Ia. SNe Ia in galaxies whose populations have a characteristic age greater than 5 Gyr are $\sim 1$ mag fainter than those found in galaxies with younger populations. Based on the evolution of the SN Ia rate with redshift, its correlation with the 


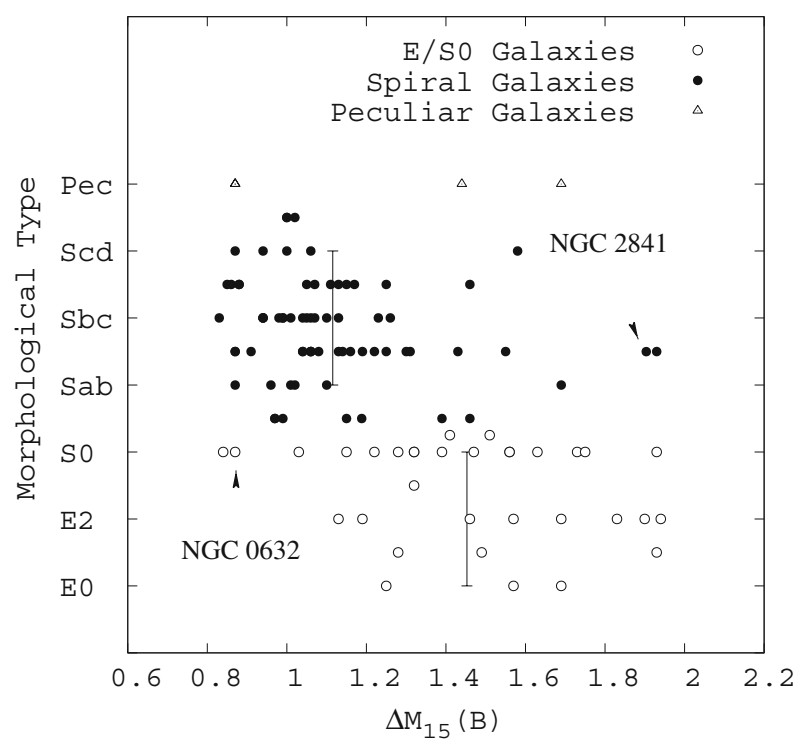

Fig. 35 A compilation of the morphological type of the host galaxy versus the decline rate of SNe Ia. NGC 2841 has spectral features of an elliptical and NGC 0632 has emission lines characteristic for latetype galaxies. The vertical markers indicate the average decline rates for early- and late-type galaxies (from Gallagher et al. 2005)

color and radio luminosity of the parent galaxies, Mannucci et al. (2006) concluded that the delay time distribution of SN Ia is bimodal. About half of the SN Ia explode $\approx 10^{8}$ years after the formation of the progenitor binary system and the delay time distribution of the other half of SN Ia could be described by an exponential function with a decay time of $\sim 3$ Gyr. This diversity should be reflected in the abundance yields. The brighter supernovae are expected to produce more $\mathrm{Ni}$ and less $\alpha$ elements ( $\mathrm{Si}, \mathrm{S}$, $\mathrm{Ar}, \mathrm{Ca}$ ) than the fainter ones.

Figure 36 shows the $\log _{10}(\mathrm{Fe} / \mathrm{Si})$ against the $\log _{10}(\mathrm{Fe} / \mathrm{O})$ for different spatial regions in clusters of galaxies Hydra A (Simionescu et al. 2009b), Sérsic 159-03 (de Plaa et al. 2006), Centaurus (Matsushita et al. 2007a), Fornax (Matsushita et al. 2007b), M 87 (Matsushita et al. 2003), and A1060 (Sato et al. 2007). The plot shows a relatively large spread in the abundance patterns determined for different clusters. In particular the differences in the Fe/Si ratio between Hydra A and M 87 are striking. Each overplotted line in Fig. 36 shows a mixture of yields of a different SN Ia model with $\mathrm{SN}_{\mathrm{CC}}$ yields by Tsujimoto et al. (1995) integrated over the Salpeter IMF. Using $\mathrm{SN}_{\mathrm{CC}}$ yields by different authors, assuming different progenitor pre-enrichment levels, or integrating the $\mathrm{SN}_{\mathrm{CC}}$ yields over a different IMF would move these curves (see Fig. 11 in Simionescu et al. 2009b). But an intrinsic variation in the metallicity or IMF of the $\mathrm{SN}_{\mathrm{CC}}$ progenitors alone could not explain the observed variety of abundance patterns. Thus it seems that, indeed, a variety of SN Ia flavors with different densities at the transition from the subsonic to supersonic flame velocities is needed.

While the ratio of the $\alpha$ elements to Fe can be different for different $\mathrm{SNe} \mathrm{Ia}$, the ratio between the $\alpha$ elements is very similar for all explosion mechanisms, because the fuel $(\mathrm{C} / \mathrm{O})$ is always the same and the burning conditions where the $\alpha$ elements 

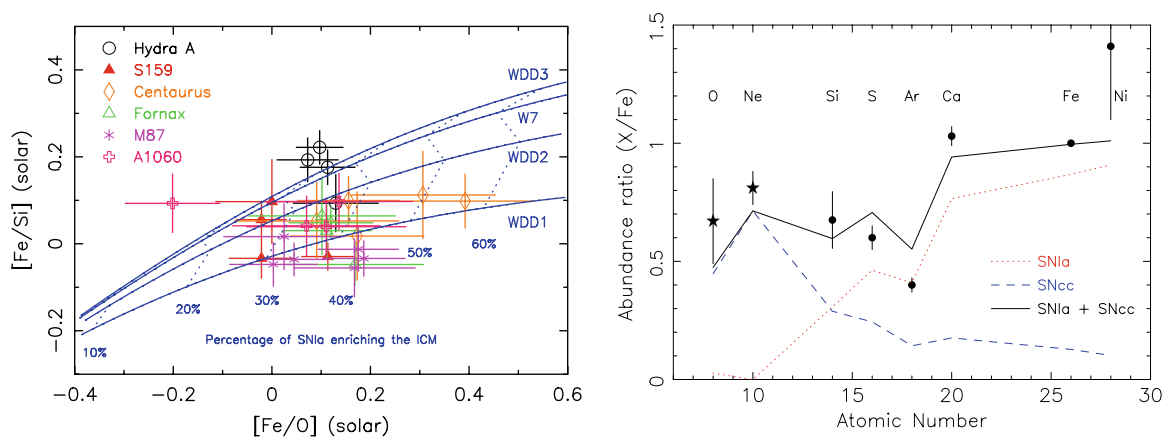

Fig. 36 Intrinsic variation of cluster abundances and their average yields. Left panel The observed [Fe/Si] versus $[\mathrm{Fe} / \mathrm{O}]$ for different spatial regions of a sample of six clusters of galaxies. The curves indicate models of a mixture of $\mathrm{SN}_{\mathrm{CC}}$ products assuming a Salpeter initial mass function (Tsujimoto et al. 1995) mixed with different SN Ia products (Iwamoto et al. 1999). The dotted line connects points of the same number contributions of SN Ia to the enrichment of the ICM. The abundance patterns in the Hydra cluster and in M 87 favor very different SN Ia models (after Simionescu et al. 2009b). Right panel Reconstruction of the average abundance patterns observed in a sample of 22 clusters of galaxies with theoretical supernova yields (from de Plaa et al. 2007). The model describing the data the best is a combination of SN Ia yields from a 1D delayed detonation model by Badenes et al. (2006) and $\mathrm{SN}_{\mathrm{CC}}$ yields for progenitors with Solar metallicities by Nomoto et al. (2006) integrated over the Salpeter IMF (from de Plaa et al. 2007)

are synthesized do not vary much in SN Ia. Therefore the relative abundances of the $\alpha$ elements, especially the $\mathrm{Ca} / \mathrm{Ar}$ ratio, are the best diagnostic of the quality of SN Ia models. de Plaa et al. (2007) analyzed the abundance patterns of Si, S, Ar, Ca, Fe, and $\mathrm{Ni}$ in a sample of 22 clusters observed with $X M M$-Newton within $0.2 R_{500}$. Because the $\mathrm{SN}_{\mathrm{CC}}$ do not have a significant impact on the Ar/Ca ratio, they used the abundances of these elements to find the best fitting SN Ia model. Delayed detonation models clearly provide a better fit to the data than the deflagration scenarios, but none of the supernova models by Iwamoto et al. (1999) fit the Ar and Ca abundances. de Plaa et al. (2007) obtained a good fit with a one-dimensional delayed-detonation SN Ia model, calculated on a grid introduced in Badenes et al. (2003), with a deflagration-todetonation density of $2.2 \times 10^{7} \mathrm{~g} \mathrm{~cm}^{-3}$ and kinetic energy of $1.16 \times 10^{51} \mathrm{ergs}$, which was shown to fit best the properties of the Tycho supernova remnant (Badenes et al. 2006).

Here it is interesting to note that Tycho seems to be a remnant of a fairly typical SN Ia with an average luminosity and the data points in the left panel of Fig. 36 also tend to be centered around the WDD2 model which is the closest to the best fitting model of Badenes et al. (2006). Several other observational clues point toward the delayed detonation models. Optical light curves (Woosley et al. 2007) and spectra (Mazzali et al. 2007), the X-ray emission of SN Ia remnants (Badenes et al. 2006, 2008) all show that the structure of the SN Ia ejecta has to be very close to what one-dimensional delayed detonation models predict: most of the white dwarf is burnt, Fe-peak nuclei stay in the inner ejecta and the intermediate mass elements stay in the outer ejecta. The variation of the peak brightness, which correlates with the production of ${ }^{56} \mathrm{Ni}$ and anti-correlates with the production of Si-group elements, can also be explained in the framework of the delayed detonation models by a variation of the 
deflagration-to-detonation transition density (transition from subsonic to supersonic flame velocities).

As shown by de Plaa et al. (2007) the relative abundances of chemical elements in the ICM can, in principle, be also used to distinguish between $\mathrm{SN}_{\mathrm{CC}}$ models with different level of pre-enrichment of the progenitors and with different initial-mass functions (IMF). Comparing the measured abundances with the $\mathrm{SN}_{\mathrm{CC}}$ yields predicted by the model of Nomoto et al. (2006), de Plaa et al. (2007) found that the dominant fraction of $\mathrm{SN}_{\mathrm{CC}}$ progenitors contributing to the enrichment of the ICM was already enriched and their mass distribution was close to the Salpeter IMF. The main constraints on the $\mathrm{SN}_{\mathrm{CC}}$ progenitors are based on the measured $\mathrm{O} / \mathrm{Ne}$ ratio in the ICM.

While elements from $\mathrm{O}$ up to the Fe-group are primarily produced in supernovae, carbon and nitrogen are believed to originate from a wide range of sources: winds of short-lived massive metal rich stars, longer lived low- and intermediate-mass stars, and an early generation of massive stars (e.g. Gustafsson et al. 1999; Chiappini et al. 2003; Meynet and Maeder 2002). The ICM abundance of C and N was measured only in a few nearby bright elliptical galaxies and clusters (Peterson et al. 2003; Werner et al. 2006a). The small $\mathrm{O} / \mathrm{Fe}$ ratio and large $\mathrm{C} / \mathrm{Fe}$ and $\mathrm{N} / \mathrm{Fe}$ found in $\mathrm{M} 87$ by Werner et al. (2006a) suggest that the main sources of $\mathrm{C}$ and $\mathrm{N}$ are not the massive stars that also produce large quantities of $\mathrm{O}$, but the low- and intermediate-mass asymptotic giant branch stars.

\subsection{Metallicity evolution and differences between clusters}

As we mentioned before, cluster data obtained by ASCA were consistent with no evolution in the ICM metallicity out to redshift $z \sim 0.4$. More recent observations with Chandra, however, indicate a significant evolution (Balestra et al. 2007; Maughan et al. 2008). Balestra et al. (2007), who investigated the Fe abundance evolution on a sample of 56 clusters, found that while in the redshift range of $z \simeq 0.3-0.5$ the average ICM Fe abundance is $\simeq 0.4$ Solar, above redshift $z \sim 0.5$ the metallicity drops to $\simeq 0.25$ Solar. Maughan et al. (2008) looked at a larger sample of 116 clusters at $0.1<z<1.3$ in the Chandra archive and essentially confirmed the results of Balestra et al. (2007). They found that the abundances drop by $\sim 50 \%$ between $z \sim 0.1$ and $z \sim 1$ and the evolution is still present if the cluster cores (the inner $0.15 R_{500}$ ) are excluded from the analysis. This result indicates that the abundance drop is not due to the lack of strong CCs at large redshifts.

Balestra et al. (2007) also found a trend of the Fe abundance with the cluster temperature. Within $(0.15-0.3) R_{\text {vir }}$ in clusters below $5 \mathrm{keV}$ the Fe abundance is on average a factor of $\sim 2$ larger than in the hotter clusters. The Fe abundance values measured within $0.2 R_{500}$ for the sample of low redshift 22 clusters analyzed by de Plaa et al. (2007) show the same trend with the cluster temperature: while in hot massive clusters $(k T \gtrsim 5 \mathrm{keV}$ ) the Fe abundance seems to be constant and equal to $\sim 0.3$ solar, for cooler clusters, in the temperature range of $2-4 \mathrm{keV}$, the Fe abundance shows a range of values between 0.2 and 0.9 solar. This trend is probably linked to the changing stellar mass over gas mass ratio in clusters. While the gas mass fraction was found to, in general, increase with the cluster temperature with a large spread of values 
especially below $5 \mathrm{keV}$ (Sanderson et al. 2003), the gas fraction in clusters above $>5 \mathrm{keV}$ is constant with the ICM temperature (Allen et al. 2008). The Fe mass over light ratio seems to be constant as a function of temperature (e.g. Renzini 1997). Lin et al. (2003) find that the stellar mass fraction decreases by a factor of 1.8 from low- to high- mass clusters, and the ICM to stellar mass ratio increases from 5.9 to 10.4. This trend suggests a decrease of star formation efficiency with increasing cluster mass and provides a natural explanation for the on average lower iron abundance in the more massive clusters. The large range of Fe abundance values in the clusters with lower temperatures might be due to the large spread in the gas mass fraction of the lower mass clusters and due to the range of enrichment times and gas densities in the CCs of clusters.

Fukazawa et al. (1998) and Baumgartner et al. (2005) showed based on ASCA data an increase of the $\mathrm{Si} / \mathrm{Fe}$ ratio from 0.7 to 3 Solar in the $2-8 \mathrm{keV}$ temperature range. This result was not confirmed by later XMM-Newton observations. The abundance ratios of $\mathrm{Si} / \mathrm{Fe}, \mathrm{S} / \mathrm{Fe}, \mathrm{Ca} / \mathrm{Fe}$, and $\mathrm{Ni} / \mathrm{Fe}$ measured within $0.2 r_{500}$ in the sample of 22 clusters with temperatures in the range of 1.5-10 keV analyzed by de Plaa et al. (2007) are consistent with being constant as a function of temperature. The intrinsic spread in the abundance ratios is smaller than $30 \%$. This result shows that the abundance patterns do not change with the observed drop of star formation efficiency with the cluster mass and the ratios of SN Ia and $\mathrm{SN}_{\mathrm{CC}}$ contributing to the ICM enrichment are similar in clusters of all masses. ${ }^{2}$

The observed drop of the Fe abundance with increasing redshift could be at least partly connected to the metallicity-temperature relation. The clusters observed at high redshift are primarily massive clusters which eventually evolve into hot clusters like those with lower abundances at low redshifts. The evolution of the metallicity below redshift $z \sim 1$ therefore might be substantially weaker than the observations suggest.

\subsection{How good are the assumptions behind the abundance measurements?}

The chemical abundances in the ICM are determined assuming a certain plasma model, a certain ICM temperature structure, optically thin plasma, and solar abundances for elements lighter than $\mathrm{O}$ or $\mathrm{N}$. How good are these assumptions and how can they bias our abundance determinations?

\subsubsection{How much bias due to spectral modeling?}

Usually two plasma models are used for fitting cluster spectra: MEKAL (Mewe et al. 1985, 1986; Kaastra 1992; Liedahl et al. 1995) and APEC (Smith et al. 2001). A more updated version of MEKAL compared to the one available in XSPEC is implemented in the SPEX spectral fitting package (Kaastra et al. 1996). MEKAL and APEC

\footnotetext{
2 The increase of the $\mathrm{Si} / \mathrm{Fe}$ ratio with the cluster temperature observed by ASCA might have been due to a bias introduced by the strong temperature gradients in the hot clusters with cooling cores. If the temperature of the best fit plasma model (the average cluster temperature) is higher than the temperature of the cooler gas with high $\mathrm{Si}$ line emissivity, then the model will naturally predict a higher $\mathrm{Si}$ abundance than the actual value.
} 

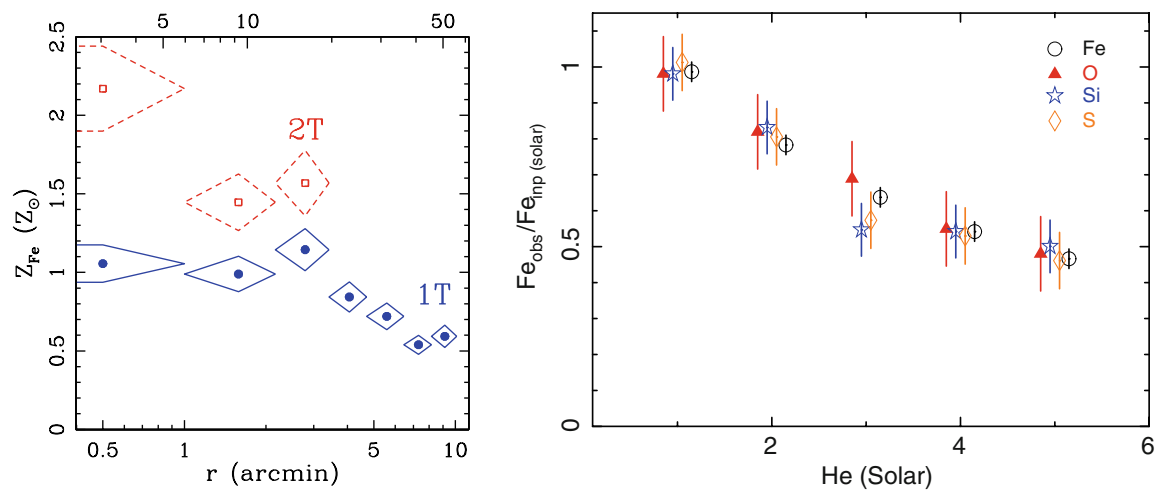

Fig. 37 Left panel The influence of the temperature model on the best fit Fe abundance as seen in the radial Fe abundance distribution in NGC 1399 modeled with single- and two-temperature models (from Buote 2002). Right panel The helium abundance is usually assumed to be Solar in clusters. If the helium abundance in cluster cores is increased by sedimentation and we do not take it into account in the spectral modeling, our best fit abundances will be lower than the real values. The effect is indicated in the plot for simulated spectra of a $4 \mathrm{keV}$ cluster. The simulation was made assuming a $100 \mathrm{ks}$ observation with $X M M-N e w t o n$ EPIC and a circular extraction region with a radius of $1^{\prime}$

treat differently the atomic physics and differences are also in their line libraries. But despite small differences in the best fit temperature structure, the abundances derived with the two plasma codes are generally consistent within the errors (Buote et al. 2003; Sanders and Fabian 2006; de Plaa et al. 2007). The two plasma codes thus do not show any systematic inconsistencies. The implemented ionization balance for $\mathrm{Ni}$ is inaccurate and can significantly bias the Ni abundance measurements; an update is planned shortly for SPEX (J. S. Kaastra, private communication).

One has to be careful and keep in mind possible biases when interpreting the results of measurements of chemical abundances in clusters. The most serious biases arise when the temperature structure in the extraction region is not modeled properly. Historically, X-ray observations showed significantly subsolar abundances in galaxies and groups of galaxies. Buote and Canizares (1994) and Buote (2000b) showed that a low resolution spectrum which is composed of intrinsically two temperature components with similar emission measures, one below $1 \mathrm{keV}$ and one above $1 \mathrm{keV}$, with an average temperature of about $1 \mathrm{keV}$ fitted with a single temperature model will seriously bias the Fe abundance determination. Since the lower temperature component preferentially excites emission lines in the Fe-L complex below $1 \mathrm{keV}$ (Fe XII-XXI) and the higher temperature component excites the Fe-L lines in the range of $\sim 1-1.4 \mathrm{keV}$ (Fe XXI-XXIV) the shape of the Fe-L complex will be flatter than that produced by a single-temperature model with the average temperature of the two components (see also Fig. 23). The single-temperature model can fit the flat shape of the Fe-L complex only with a relatively low Fe abundance and higher bremsstrahlung continuum. The obtained Fe abundance is thus much lower than the actual value (see the left panel of Fig. 37).

In the past few years several authors (e.g. Buote et al. 2003; Werner et al. 2006b; Matsushita et al. 2007a) showed that in the complex CCs of groups and clusters the obtained abundances of essentially all elements increase when two-temperature 
models or more complicated differential emission measure (DEM) models are used instead of simple single-temperature models.

For a multi-temperature plasma with average temperatures of $\sim 2-4 \mathrm{keV}$, where both $\mathrm{Fe}-\mathrm{L}$ and $\mathrm{Fe}-\mathrm{K}$ emission lines are seen with similar statistics, fitted with a single temperature model an "inverse Fe bias" is observed. Simionescu et al. (2009b) point out that in a multi-temperature plasma at these temperatures the cool component produces stronger Fe-L lines and the hotter component emits more Fe-K emission than that expected for the single-temperature plasma with the average temperature. Therefore, by fitting a single temperature model to such plasma one will obtain a higher Fe abundance than the actual value (see also Rasia et al. 2008).

These results show that for chemical abundance studies in the ICM a good description of the temperature structure is crucial. Accurate abundance determinations require deep observations which allow to test different differential emission measure distributions to obtain the best description of the true temperature structure in the cluster cores.

\subsubsection{Possible biases due to helium sedimentation?}

In the gravitational potential of clusters helium and other metals can diffuse toward the cluster center (Fabian and Pringle 1977; Abramopoulos et al. 1981; Gilfanov and Syunyaev 1984). The sedimentation is more efficient for the lighter elements and in a $\mathrm{H}-\mathrm{He}$ plasma, the helium is expected to be more centrally peaked than hydrogen. The helium abundance cannot be directly measured. The cluster spectra are commonly modeled under the assumption of solar or primordial He abundance values in the ICM. In the inner regions of galaxy clusters where the effects of sedimentation are the most important, the underestimation of the amount of helium can cause inaccurate determination of gas densities and metallicities.

Peng and Nagai (2009) solved a set of flow equations for a H-He plasma for clusters assuming temperature profiles by Vikhlinin et al. (2006). They showed that at $0.06 r_{500}$ of a $11 \mathrm{Gyr}$ massive $(10 \mathrm{keV})$ cluster the $\mathrm{He} / \mathrm{H}$ abundance ratio can be as high as 4.3 Solar. These predictions are, however, made for unmagnetized plasma assuming no turbulent suppression of particle diffusion and no mixing in the ICM, therefore they should be treated as upper limits. Ettori and Fabian (2006) pointed out that for an actual helium abundance of $\sim 4$ times the value assumed in the spectral modeling, would cause an under-determination of the Fe abundance by a factor of 2 . As we show in the right panel of Fig. 37 the same is approximately true for the other elements as well.

\subsubsection{Resonance scattering in cooling cores?}

For the strongest resonant lines the ICM can be moderately optically thick (Gilfanov et al. 1987) and as we already mentioned in Sect. 3.5 resonant scattering at the energies of these lines changes the observed line intensities. This can affect the measurements of elemental abundances in the dense cores of clusters. 
Sanders and Fabian (2006) constructed a model that takes into account resonant scattering for thousands of resonance lines. They tested their model on Chandra data of two clusters with central abundance dips. They conclude that metallicities in cluster cores could be underestimated due to resonant scattering by at most $10 \%$. Resonance scattering can therefore not be the cause for the observed central abundance dips.

In order to determine the amount of resonant scattering Werner et al. (2009) studied the line ratios of the Fe XVII ion in 5 elliptical galaxies (see Sect. 3.5). They concluded that neglecting the effects of resonant scattering in the spectral fitting of the inner $2 \mathrm{kpc}$ core of NGC 4636 will lead to underestimates of the chemical abundances of Fe and $\mathrm{O}$ by $\sim 10-20 \%$.

\section{Beyond thermal equilibrium}

The intra-cluster medium is usually assumed to be in collisional ionization equilibrium (CIE). Plasma in CIE is optically thin for its own radiation and external radiation fields do not affect the ionization balance which is entirely determined by the temperature of the plasma. The ionization and recombination rates in CIE plasma have come to a balance and the electron and ion temperatures are in equilibrium. The energy distribution of the electrons in the ICM is usually described by a Maxwellian.

These assumptions are mostly justified but there are certain situations when the plasma deviates from the thermal equilibrium. The shock heated plasma in the cluster outskirts with densities $n_{e} \lesssim 10^{-4} \mathrm{~cm}^{-3}$ is likely to be out of ionization balance. The equilibration time scale at those low densities is longer than the age of the cluster and therefore the gas may still ionize. At the lowest density parts of warm-hot intergalactic medium filaments photoionization by the diffuse radiation field of galaxies and the cosmic background becomes important. Deviations from a Maxwellian electron distribution could occur in the cooling cores of clusters where multiple gas phases are present. If electrons diffuse from a hotter into a cooler phase they over-ionize the gas. Plasma could also be out of equilibrium in the vicinity of shocks, which may alter the electron/ion equilibrium. Shocks could also accelerate electrons and produce non-Maxwellian tails in the ICM electron distribution. Future observatories with large photon collecting area and high spectral resolution will be able to study the spectral signature of these non-equilibrium effects in detail. Moreover, radio observations of clusters reveal the presence of relativistic electrons emitting synchrotron radio emission. This relativistic plasma in clusters may also emit Inverse-Compton radiation in the form of a power law shaped X-ray continuum.

Below we briefly describe what are the spectral signatures of non-equilibrium effects in the multiphase plasma and around shocks, and we summarize the state of observations of X-ray emission from relativistic plasma.

\subsection{Multiphase plasma}

High signal-to-noise X-ray spectra reveal that multiple spatially unresolved gas phases are present in the cooling cores of clusters (Kaastra et al. 2004; Sanders and Fabian 2007; Simionescu et al. 2008). In M87 and Perseus, the most nearby and the brightest 


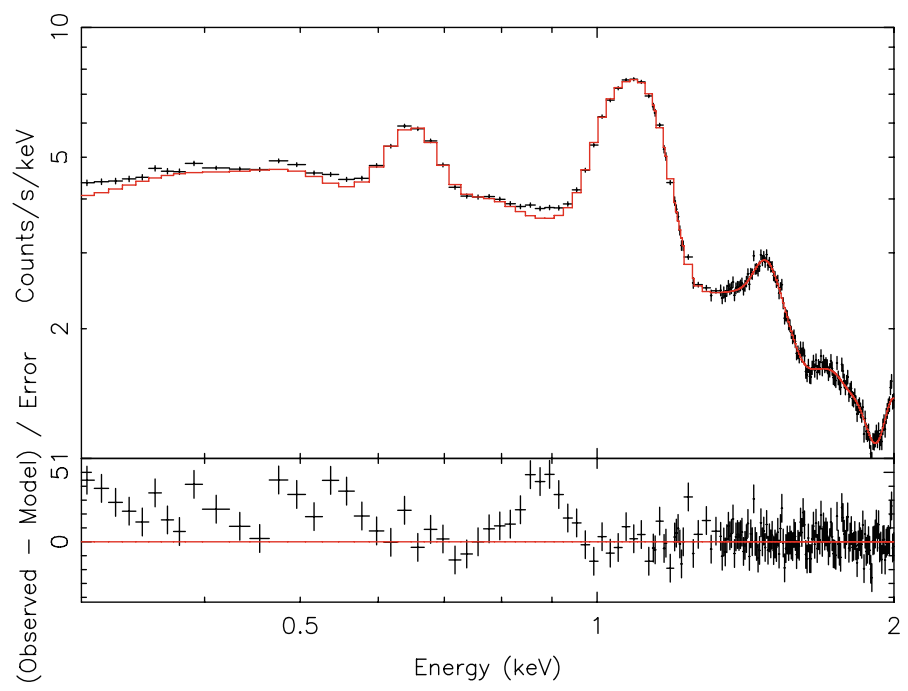

Fig. 38 Simulated EPIC/pn spectrum of the Eastern X-ray arm of M87 assuming a presence of a filament with a diameter of $60 \mathrm{pc}$ and length of $3.5 \mathrm{kpc}$ filled with $10^{4} \mathrm{~K}$ gas with an average density of $n_{e}=10 \mathrm{~cm}^{-3}$. We assumed that $0.05 \%$ of all the electrons within the filament penetrated there from the ambient ICM and have a Maxwellian distribution with a temperature of $2 \mathrm{keV}$. The model plotted with the red line shows the emission from the ambient hot ICM. Assuming a well calibrated instrument the excess line emission at $\sim 0.85 \mathrm{keV}$ would be clearly revealed

cooling cores, images obtained by Chandra reveal filaments and blobs in soft X-rays (Forman et al. 2007; Fabian et al. 2006). But the multi-phase extends to much lower temperatures. Narrow band optical observations of cooling cores reveal $\mathrm{H} \alpha$ emitting warm $\left(10^{4} \mathrm{~K}\right)$ gas which both in M87 and in Perseus is often associated with soft X-ray filaments (Sparks et al. 2004; Fabian et al. 2006). The H $\alpha$ filaments in Perseus are composed of gas with temperatures of $10^{4} \mathrm{~K}$ down to $50 \mathrm{~K}$ (Hatch et al. 2005). Sanders and Fabian (2007) showed that they are surrounded and possibly mixed with soft X-ray emitting plasma with temperatures of $0.5-1 \mathrm{keV}$.

If electrons penetrate from the hotter to the cooler phase they over-ionize the "cool" plasma which may result in observable low energy X-ray line emission. In Fig. 38 we show a simulated EPIC/pn spectrum $(100 \mathrm{ks})$ of the Eastern X-ray arm of M87. Within this extraction region, we assume the presence of a filament with a diameter of $60 \mathrm{pc}$ and length of $3.5 \mathrm{kpc}$ filled with $10^{4} \mathrm{~K}$ gas with an average density of $n_{e}=10 \mathrm{~cm}^{-3}$. We assume that $0.05 \%$ of all the electrons within the filament have penetrated there from the ambient ICM and have a Maxwellian energy distribution with a temperature of $2 \mathrm{keV}$. As the spectrum in Fig. 38 shows, a deep observation with a well calibrated instrument would even at medium spectral resolution reveal the line emission from Ne III-VI at $0.85-0.9 \mathrm{keV}$, O IV-VII at $\sim 0.55 \mathrm{keV}, \mathrm{N}$ III-VI between 0.40 and $0.42 \mathrm{keV}, \mathrm{C}$ IV-V at $0.35,0.37 \mathrm{keV}$, and at $0.30-0.31 \mathrm{keV}$. This exercise demonstrates the potential of X-ray spectroscopy in constraining the diffusion of electrons from the hot ICM phase into the warm filaments. In reality such attempts are hampered by the complicated multi-temperature structure of the ICM around the filaments. Detailed studies of the electron diffusion will be possible with future micro-calorimeters or transition-edge sensors sensitive at low energies. 

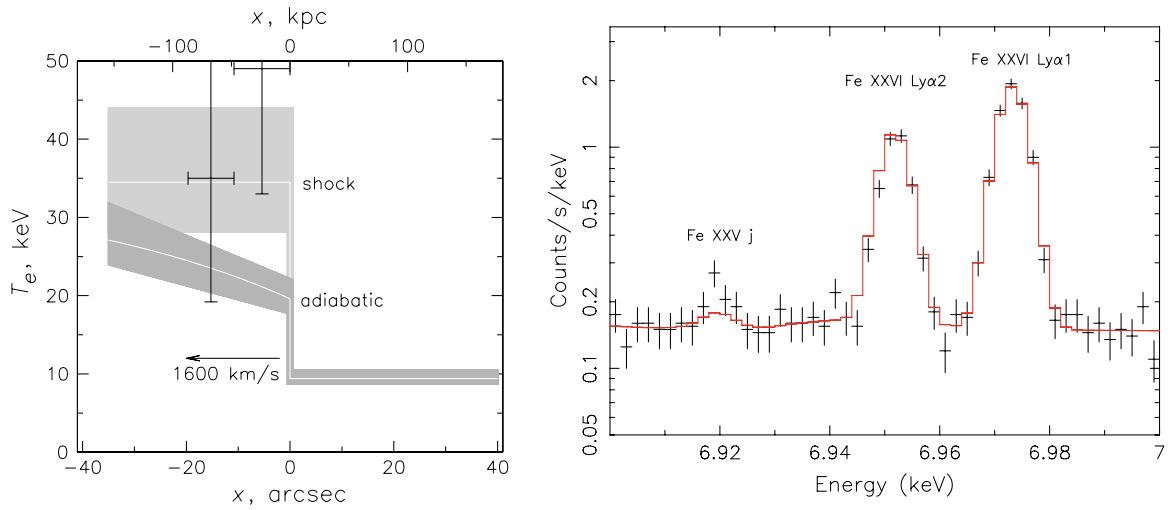

Fig. 39 Left panel Electron-ion equilibration in 1E 0657-56 (the "Bullet cluster"). The two data points show the deprojected electron temperature for two narrow post shock regions. The data points are overlaid on the model predictions for instant equilibration (light gray) and for adiabatic compression followed by collisional equilibration (dark gray). The velocity of the post-shock gas relative to the shock front is $\sim 1,600 \mathrm{~km} \mathrm{~s}^{-1}$ (from Markevitch 2006). Right panel The 6.9-7.0 keV part (rest frame energies) of a simulated Astro- $H$ micro-calorimeter spectrum of the downstream region of a $M=2.2$ shock propagating through a $10^{8} \mathrm{~K}$ plasma. The Fe XXVJ satellite line is clearly stronger (with a significance of $3 \sigma$ for these simulations) than that predicted by the thermal model with a Maxwellian electron distribution indicated by the full line (from Kaastra et al. 2009)

\subsection{Shocks and non-equilibrium}

In the downstream regions of shock fronts the electron and ion temperatures of the plasma might be different and the ICM is out of ionization equilibrium.

In a collisional plasma, protons are heated dissipatively at the shock layer which has approximately the width of the collisional mean free path, but the faster moving electrons do not feel the shock (for Mach numbers smaller than $~ 40$ ), they get adiabatically compressed and equilibrate with the ions via Coulomb collisions reaching the post-shock temperature predicted by the Rankine-Hugoniot jump condition. Astrophysical shocks in a magnetized plasma, like the ICM, are expected to be "collisionless". Which means that the electron and ion temperature jump occurs on a spatial scale much smaller than the collisional mean free path, but ions and electrons are heated by the shock to different temperatures. In heliospheric shocks with moderate Mach numbers electrons are heated much less than protons, barely above the adiabatic compression temperature (Schwartz et al. 1988). The plasma behind the shocks is thus expected to be in electron/ion temperature non-equilibrium. Assuming that no kinetic energy goes into acceleration of cosmic rays, the post-shock temperature after the Coulomb equilibration time scale reaches the same temperature as the one predicted by the Rankine-Hugoniot jump condition.

Markevitch (2006) used the bow shock in the "Bullet cluster" (1E 0657-56) to determine whether the electrons in the intra-cluster plasma are heated directly at the shock to the equilibrium temperature (instant-equilibration), or whether they equilibrate via Coulomb collisions with protons. They measure the temperature in front of the shock front seen as a gas density jump, from which, using the adiabatic and RankineHugoniot jump conditions, they determine the expected post shock adiabatic and 
instant-equilibration electron temperatures which they directly compare with observed data. The shock in the "Bullet cluster" is happening approximately in the plane of the sky, which means that the downstream velocity of the shocked gas spreads out the time dependence of the electron temperature in the plane of the sky. The measured temperatures behind the shock in the bullet cluster are consistent with instant heating of electrons. Adiabatic compression followed by equilibration on the collisional time scale is excluded at the 95\% confidence level (see Fig. 39).

As the temperature of the plasma suddenly rises at a shock front, it gets out of ionization balance. While the ionization state of ions still reflects the pre-shock temperature of the plasma after the instant heating, the electron temperature is higher. The plasma is under-ionized compared to the equilibrium case and the ionization balance must be recovered by collisions. Until the ionization balance recovers there will be more ionization in the plasma than recombination. The exact ionization state of shocked plasma in non-equilibrium conditions depends on its temperature, density, and the time since it has been shocked. Such situation is often seen in supernova remnants.

Shocks and turbulence in the magnetized ICM are believed to be sides of nonthermal particle acceleration in clusters (for reviews on particle acceleration see, e.g. Petrosian and Bykov 2008; Bykov et al. 2008). Stochastic turbulence in the ICM can create a non-thermal tail to the Maxwellian electron distribution (Bykov and Uvarov 1999). The low energy end of the power-law electron distribution enhances the ionization rates and modifies the degree of the ionization of the plasma. Porquet et al. (2001) showed that the plasma is always more ionized for hybrid (Maxwellian plus powerlaw) electron distribution than for a Maxwellian distribution and the mean charge of a given element at a given temperature is increased. The effect is more pronounced at lower temperatures. In groups of galaxies, low mass clusters or in cooling cores a peculiar ionization state of Fe which is inconsistent with the temperature determined from continuum emission can potentially be a good tool to reveal such non-thermal tails in the electron distribution. Kaastra et al. (2009) show that a non-Maxwellian tail in the electron distribution behind a shock front propagating through $10^{8} \mathrm{~K}$ plasma cannot be revealed by current detectors. They simulate the electron distribution and the resulting X-ray spectra of the downstream region of a $M=2.2$ shock in a X-ray bright hot cluster and show that a good indicator of hard non-thermal electrons is the enhancement of the equivalent widths of satellite lines which may be possible to detect with the X-ray micro-calorimeters on the future Astro- $H$ satellite or on the proposed International X-ray Observatory (IXO). In the right panel of Fig. 39, we show the 6.9-7.0 keV part (rest frame energies) of a simulated $100 \mathrm{ks}$ Astro- $H$ spectrum of the downstream region of a $M=2.2$ shock propagating through the ICM in a cluster similar to Abell 2029. The Fe XXVI Ly $\alpha$ lines and the Fe XXVJ satellite line are visible in the spectrum and the satellite line is clearly stronger than that predicted by the thermal model with a Maxwellian electron distribution indicated by the full line.

\subsection{Non-thermal X-ray emission from relativistic plasma}

Direct evidence for the presence of electrons accelerated up to relativistic energies comes from observations of radio emission in clusters. Large-scale diffuse extended 
radio emission in the form of halos or relics has been observed in about 50 known clusters of galaxies (Feretti and Giovannini 2008). This radiation is associated with the ICM and has no connection to the cluster galaxies. It is clearly produced by synchrotron emission by a population of relativistic electrons in the ICM. Since the energy density of the cosmic microwave background (CMB) radiation $(\sim 4 \times$ $\left.10^{-13} \mathrm{erg} \mathrm{cm}^{-3}\right)$ is higher than the energy density in the intra-cluster magnetic field $\left(3 \times 10^{-14}(B / \mu \mathrm{G})^{2} \mathrm{erg} \mathrm{cm}^{-3}\right)$, these relativistic electrons will radiate away most of their energy via inverse-Compton scattering of the CMB photons, producing a powerlaw shaped X-ray continuum emission (Rephaeli 1977). The detection of both the non-thermal X-ray emission and the radio emission would be a powerful tool, which would allow us to determine both the volume averaged magnetic field in clusters and the energy in the population of relativistic electrons. But for the observed radio fluxes, detectable hard X-ray emission can only be produced if the magnetic fields are of the order of $0.1 \mu \mathrm{G}$. Faraday rotation measurements indicate that the intra-cluster magnetic fields are much stronger - of the order of $1-10 \mu \mathrm{G}$. The volume averaged magnetic field might, however, be weaker than the strong magnetic field measured by Faraday rotation along our line of sight.

Non-thermal X-ray emission could in principle also originate in non-thermal bremsstrahlung of shock accelerated supra-thermal electrons in the ICM. However, such non-thermal bremsstrahlung phase must be very short lived. Petrosian (2001) points out that in order to explain the reported hard X-ray luminosity in the Coma cluster (see later) by bremsstrahlung emission, the continuous input of energy into the ICM would increase the ICM temperature to $10^{10} \mathrm{~K}$ in Hubble time. Also the high-energy electrons $>50 \mathrm{keV}$ cannot be confined by the gravitational potential of the cluster and will escape in a crossing time of $<1.5 \times 10^{7}$ years, unless they are confined by the intracluster magnetic field. Alternatively, hard X-ray emission with power-law spectra of $\Gamma \sim 1.5$ could be produced by synchrotron emission from ultra-relativistic electrons and positrons produced by the interaction of relativistic protons with the CMB (Inoue et al. 2005).

The nearest hot massive cluster of galaxies with a big radio halo is the Coma cluster (Feretti and Giovannini 1998). Therefore, Coma has been the primary target to identify non-thermal X-rays. Their detection has been reported with both BeppoSAX (Fusco-Femiano et al. 1999) and RXTE (Rephaeli and Gruber 2002). More recently, Coma has also been observed with the INTEGRAL satellite (Eckert et al. 2007). A combined analysis of XMM-Newton and INTEGRAL data revealed the presence of hotter $(\sim 12 \mathrm{keV})$ gas in the south-west region overlapping with a radio halo. This hot gas was probably heated in a merger (Eckert et al. 2007). Analyzing INTEGRAL, ROSAT, and RXTE data, Lutovinov et al. (2008) found that the global Coma spectrum is well fitted with a thermal model and the evidence for a hard excess is very marginal $(1.6 \sigma)$. Ajello et al. (2009) analyzed combined XMM-Newton and hard X-ray Swift-BAT spectra and even though they could not rule out the presence of non-thermal hard X-ray emission outside of their $10^{\prime}$ region, within their field of view they found a good fit with two thermal models, with no need for a non-thermal component. Wik et al. (2009) analyzed combined Suzaku Hard X-ray Detector (HXD-PIN) and XMMNewton mosaic spectra of a larger $34^{\prime} \times 34^{\prime}$ region centered on the Coma cluster. They 
found no statistically significant evidence for non-thermal emission implying a lower limit of $0.15 \mu \mathrm{G}$ on the cluster averaged magnetic field.

Suzaku observations of the bright radio relic in the merging cluster Abell 3667 show that, at least in some cases, magnetic fields can be high even at distances of $\sim 1 \mathrm{Mpc}$ from the cluster core. The upper limits on the non-thermal emission from Suzaku put a lower limit of $2.3 \mu \mathrm{G}$ on the magnetic field in the radio relic. The non-thermal energy density in the relics is $>7 \%$ of the thermal energy density and likely near $20 \%$ (Nakazawa et al. 2009).

Ajello et al. (2009) analyzed the spectra of 10 clusters of galaxies detected above $15 \mathrm{keV}$ with the Swift-BAT. Except of the Perseus cluster the spectrum of which is probably contaminated by hard X-ray emission from the central AGN, the spectra of all clusters are well fitted by a simple thermal model. Their stacked spectrum of 8 clusters (except Perseus and Coma) also confirms the absence of any non-thermal high energy component down to a flux of $1.9 \times 10^{-12} \mathrm{erg} \mathrm{cm}^{-2} \mathrm{~s}^{-1}$ in the $50-100 \mathrm{keV}$ band.

Although there have been several other reports claiming the detection of both hard and soft X-ray emission in excess to the ICM emission, interpreted as being possibly of non-thermal origin (for reviews see Rephaeli et al. 2008; Durret et al. 2008), a solid identification of non-thermal X-rays from clusters is still lacking. In the relatively near future, X-ray satellites with imaging hard X-ray optics sensitive up to $40 \mathrm{keV}$ will be launched: Astro-H, NuSTAR, Simbol-X. They will shed more light on the presence of very hot thermal gas, non-thermal and supra-thermal electron populations, and on intra-cluster magnetic fields. The Fermi Gamma- ray Large Array Space Telescope (GLAST) might detect gamma ray emission associated with relativistic intra-cluster ions. These missions promise a big progress in our understanding of the non-thermal particle population in clusters of galaxies.

\section{Future potential of X-ray spectroscopy}

One of the most awaited features of the future of X-ray observatories is high resolution spectroscopy of the next generation of imaging devices based on calorimetric measurements of the photon energy in detectors cooled to few tens of mK. The two missions for which such detectors are envisioned and actually play a major role in the scope of these missions are Astro-H in Japan (with a possible launch in $2013^{3}$ ) and the International X-ray Observatory (IXO) currently discussed by ESA, NASA, and JAXA as a mission for about 2018-2021. ${ }^{4}$ While Astro-H (Takahashi et al. 2009) is already being built, the $I X O$ mission has not yet been fully approved and is still in the first phase of technical studies. Nevertheless, to give an outlook on the coming spectroscopic capabilities of these future X-ray missions, we will use the current prospective response files for the IXO mission with the planned so called narrow field detectors (NFI), which provide high resolution spectroscopy of about $2-3 \mathrm{eV}$ energy resolution, to illustrate the prospects. In addition to providing high resolution spectroscopy

\footnotetext{
${ }^{3}$ http://astro-h.isas.jaxa.jp/.

${ }^{4}$ http://ixo.gsfc.nasa.gov/.
} 


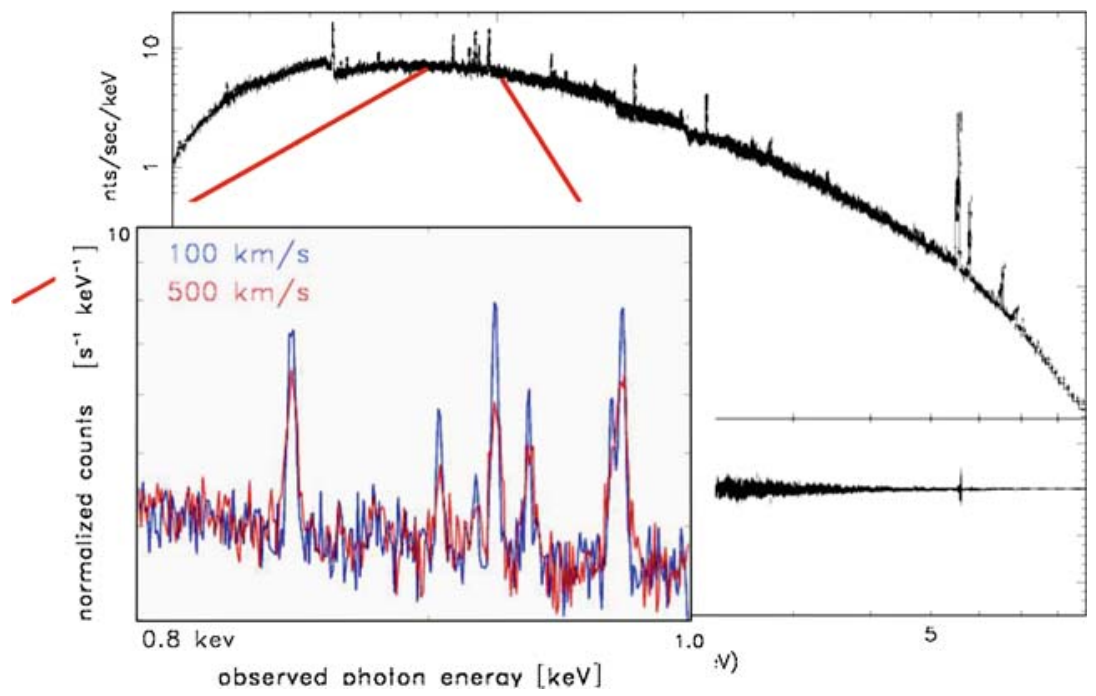

Fig. 40 Simulated $I X O$ NFI spectrum of cluster emission with a flux of $3 \cdot 10^{-13} \mathrm{erg} \mathrm{s}^{-1} \mathrm{~cm}^{-2}$ in the NFI field-of-view at a redshift $z \sim 0.2$ with an exposure of $100 \mathrm{ks}$. The inset shows the spectral structure of major Fe L-shell lines around $1 \mathrm{keV}$. Spectral line broadening for plasma with mean turbulent velocities of 100 and $500 \mathrm{~km} / \mathrm{s}$ are compared in this inset

IXO will also significantly increase the photon collecting power compared to XMMNewton with a proposed collecting area around $1 \mathrm{keV}$ of about $3 \mathrm{~m}^{2}$ to provide a better photon statistics to fully exploit the higher spectral resolution statistically. It will also increase the redshift range of cluster studies providing a detailed picture of galaxy cluster astrophysics up to redshifts of 2.

With the prospective energy resolution it will be possible to trace gas motions in the ICM with an accuracy of better than $100 \mathrm{~km} \mathrm{~s}^{-1}$ in the Fe lines in the X-ray spectra. This is made possible due to the fact that the thermal line broadening for lines from $\mathrm{Fe}$ ions is smaller by a factor of about 7 than the sound velocity which corresponds closely to the thermal velocity of the bulk of the baryons in the ICM, the protons. Figure 40 shows the simulated spectrum as observed with the IXO NFI of a patch in a cluster with an aperture radius of 1 arcmin and a flux of $3 \times 10^{-13} \mathrm{erg} \mathrm{s}^{-1} \mathrm{~cm}^{-2}$ (at $0.5-$ $2 \mathrm{keV}$ ). With an exposure of about $100 \mathrm{ks}(40 \mathrm{ks})$ a Gaussian shape turbulent velocity broadening can be measured with an accuracy of $40(70) \mathrm{km} \mathrm{s}^{-1}$. Even for a cluster at redshift 1 with a flux of $10^{-14} \mathrm{erg} \mathrm{s}^{-1} \mathrm{~cm}^{-2}$ velocity broadening accuracy would still be $100 \mathrm{~km} \mathrm{~s}^{-1}$ in an exposure of $100 \mathrm{ks}$. This will provide many new insights into the ICM physics, answering questions such as for example: how much turbulent energy is injected into the ICM by the interaction of CCs with the central AGN? How much of the $\mathrm{CC}$ heating comes from the dissipation of this turbulent energy? How is the energy dissipated in merging clusters and how is this reflected in the turbulent and bulk motion structure of the ICM? How much can the mass estimate of clusters be improved if we can measure the energy content of the ICM in turbulent energy?

Finally, Fig. 41 illustrates the potential of IXO to provide detailed information on very distant clusters. Common objects at redshift 2 having a sky density of about 1 per 


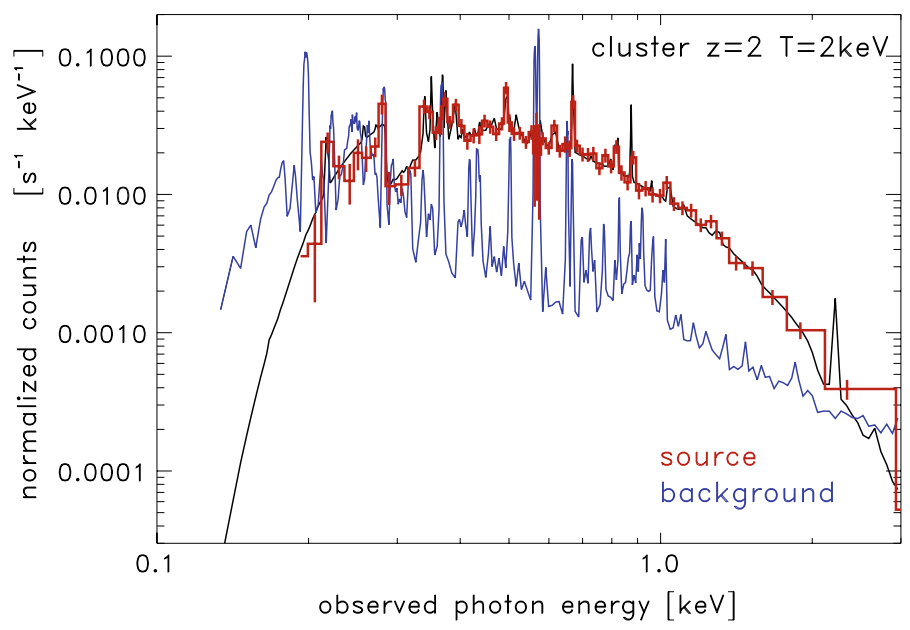

Fig. 41 Simulated IXO NFI spectrum of a galaxy group with an ICM temperature of $2 \mathrm{keV}$ at redshift 2. Also shown is the estimated background spectrum. ICM properties like the temperature and abundance measurements can well be determined from this simulated exposure of $250 \mathrm{ks}$. See text for details

$\operatorname{deg}^{2}$ are poor clusters with masses of about $3 \times 10^{13} \mathrm{M}_{\odot}$ and probably temperatures around $2 \mathrm{keV}$. Figure 41 shows the a spectrum of such an object observed with the IXO NFI instrument for $250 \mathrm{ks}$ (see alsoArnaud et al. 2009). It will allow the measurement of the bulk temperature with an accuracy better than $5 \%$ and provide abundance determinations with interesting precision: $\mathrm{Fe}( \pm 11 \%), \mathrm{Si}( \pm 18 \%), \mathrm{O}, \mathrm{Mg}( \pm 30 \%)$. This will allow us to study the thermal and chemical enrichment history of galaxy clusters over most of the interesting cosmic epochs in which clusters existed. For the more nearby bright clusters $I X O$ will very accurately measure the abundances of many chemical elements, including trace elements like $\mathrm{Cr}$, Ti, Mn, or Co. The other elements, which we detect in clusters with XMM-Newton and Chandra (O, Ne, Mg, Si, S, Ar, Ca, Fe, Ni) will be traced with an unprecedented accuracy, which will help us to put better constrains on supernova yields and thus on their explosion mechanisms. Furthermore, the wide field imager on $I X O$ will allow us to resolve the 2-dimensional metal distribution down to the relevant mixing physical scales and study in detail the process of metal injection.

The filamentary warm-hot intergalactic medium (WHIM) permeating the cosmic web might contain up to $50 \%$ of baryons at redshifts of $z \lesssim 2$. This intergalactic gas is heated to temperatures between $10^{5}$ and $10^{7} \mathrm{~K}$ as it gathers in regions of overdensity 10-100 (with respect to the mean baryon density of the Universe; at the current epoch the mean baryon density is $\left\langle n_{b}\right\rangle=2 \times 10^{-7} \mathrm{~cm}^{-3}$ ). Because this gas has such a low density and because it is so highly ionized, it is very hard to detect. The current instrumentation only allows us to probe the highest overdensity regions of the WHIM, such as the filament between the clusters Abell 222 and 223 (Werner et al. 2008), or to look for absorption features toward bright continuum sources in sight lines with known large scale structure, such as H2356-309 behind the Sculptor Wall (Buote et al. 2009). The combination of the large collecting area of $I X O$ and the high spectral resolution of its diffraction grating spectrometers will allow us to begin to probe the low-overdensity 
regions of the intergalactic medium. High-resolution X-ray absorption spectroscopy of sight lines toward bright continuum sources may allow us to probe overdensities of 10 or more (assuming a metallicity of 0.1 Solar), through the absorption features of $\mathrm{H}$ - and He-like ions of oxygen. Emission line imaging spectroscopy of the WHIM should be pursued with a large field-of-view (of order one degree), short focal length, large grasp (effective area times field-of-view) soft X-ray telescope, with a high resolution imaging X-ray spectrometer at the focus. A successful experiment will need a grasp of order several hundred $\mathrm{cm}^{2} \mathrm{deg}^{2}$, and a spectral resolution of order $1-2 \mathrm{eV}$ or better (Paerels et al. 2008).

Apart from these expectations, a new mission like $I X O$ capable of opening a new parameter space for X-ray spectroscopy and simultaneously increasing the sensitivity substantially and thus the reach of the observations, has an enormous potential for new discoveries. The most important discoveries of such missions generally come as surprises. Since the concept of IXO is already realistic - apart from detailed technical problems still to be solved-and also not much more costly than previous missions, we can expect its realization. Therefore we can foresee a bright future for X-ray spectroscopic research also over the coming two decades, and clusters will surely be among the most interesting objects to study.

Acknowledgments We like to thank A. Bykov, G. Pratt, Carles Badenes, and Aurora Simionescu for a critical reading of the manuscript and for discussions. NW acknowledges support provided by the National Aeronautics and Space Administration through Chandra Postdoctoral Fellowship Award Number PF890056 issued by the Chandra X-ray Observatory Center, which is operated by the Smithsonian Astrophysical Observatory for and on behalf of the National Aeronautics and Space Administration under contract NAS8-03060. HB acknowledges support for the research group through The Cluster of Excellence "Origin and Structure of the Universe", funded by the Excellence Initiative of the Federal Government of Germany, EXC project number 153 and support from the DfG Transregio Programme TR33.

Open Access This article is distributed under the terms of the Creative Commons Attribution Noncommercial License which permits any noncommercial use, distribution, and reproduction in any medium, provided the original author(s) and source are credited.

\section{References}

Abramopoulos F, Chanan GA, Ku WHM (1981) On the equilibrium distribution of the elements in the gas in the Coma cluster. ApJ 248:429-438. doi:10.1086/159168

Ajello M, Rebusco P, Cappelluti N, Reimer O, Böhringer H, Greiner J, Gehrels N, Tueller J, Moretti A (2009) Galaxy clusters in the swift/burst alert telescope era: hard X-rays in the intracluster medium. ApJ 690:367-388. doi:10.1088/0004-637X/690/1/367. arXiv:0809.0006

Akritas MG, Bershady MA (1996) Linear regression for astronomical data with measurement errors and intrinsic scatter. ApJ 470:706-714. doi:10.1086/177901. arXiv:astro-ph/9605002

Allen SW, Fabian AC (1998) The relationship between cooling flows and metallicity measurements for X-ray-luminous clusters. MNRAS 297:L63-L68. arXiv:astro-ph/9802219

Allen SW, Fabian AC, Johnstone RM, Arnaud KA, Nulsen PEJ (2001) ASCA and ROSAT observations of nearby cluster cooling flows. MNRAS 322:589-613. doi:10.1046/j.1365-8711.2001.04135.x. arXiv:astro-ph/9910188

Allen SW, Rapetti DA, Schmidt RW, Ebeling H, Morris RG, Fabian AC (2008) Improved constraints on dark energy from Chandra X-ray observations of the largest relaxed galaxy clusters. MNRAS 383:879-896. doi:10.1111/j.1365-2966.2007.12610.x. arXiv:0706.0033

Anders E, Grevesse N (1989) Abundances of the elements-meteoritic and solar. Geochim Cosmochim Acta 53:197-214 
Arnaud M, Rothenflug R (1985) An updated evaluation of recombination and ionization rates. A\&AS 60:425-457

Arnaud M, Rothenflug R, Boulade O, Vigroux L, Vangioni-Flam E (1992) Some constraints on the origin of the iron enriched intracluster medium. A\&A 254:49-64

Arnaud M, Pointecouteau E, Pratt GW (2005) The structural and scaling properties of nearby galaxy clusters. II. The M-T relation. A\&A 441:893-903. doi:10.1051/0004-6361:20052856. arXiv:astro-ph/0502210

Arnaud M, Pointecouteau E, Pratt GW (2007) Calibration of the galaxy cluster M $\{500\}-Y\{X\}$ relation with XMM-Newton. A\&A 474:L37-L40. doi:10.1051/0004-6361:20078541. arXiv:0709.1561

Arnaud M, Barcons X, Barret D, Bautz M, Bellazzini R, Bleeker J, Böhringer H, Boller T, Brandt WN, Cappi M, Carrera F, Comastri A, Costa E, Courvoisier T, de Korte P, Dwelly T, Fabian A, Flanagan K, Gilli R, Griffiths R, Hasinger G, Kaastra J, Kahn S, Kelley R, Kunieda H, Makishima K, Matt G, Mendez M, Mitsuda K, Nandra K, Ohashi T, Page M, Palumbo G, Pavlinsky M, Sciortino S, Smith A, Strüder L, Takahashi T, Türler M, Turner M, Ueda Y, Vignali C, Vink J, Warwick R, Watson M, Willingale R, Zhang SN (2009) XEUS: the physics of the hot evolving universe. Exp Astron 23:139168. doi:10.1007/s10686-008-9104-y

Badenes C, Bravo E, Borkowski KJ, Domínguez I (2003) Thermal X-ray emission from shocked ejecta in type Ia supernova remnants: prospects for explosion mechanism identification. ApJ 593:358-369. doi:10.1086/376448. arXiv:astro-ph/0304552

Badenes C, Borkowski KJ, Hughes JP, Hwang U, Bravo E (2006) Constraints on the physics of type Ia supernovae from the X-ray spectrum of the tycho supernova remnant. ApJ 645:1373-1391. doi:10. 1086/504399. arXiv:astro-ph/0511140

Badenes C, Hughes JP, Cassam-Chenaï G, Bravo E (2008) The persistence of memory, or how the X-ray spectrum of SNR 0509-67.5 reveals the brightness of its parent type Ia supernova. ApJ 680:1149-1157. doi:10.1086/524700. arXiv:0801.4761

Balestra I, Tozzi P, Ettori S, Rosati P, Borgani S, Mainieri V, Norman C, Viola M (2007) Tracing the evolution in the iron content of the intra-cluster medium. A\&A 462:429-442. doi:10.1051/0004-6361: 20065568. arXiv:astro-ph/0609664

Baumgartner WH, Loewenstein M, Horner DJ, Mushotzky RF (2005) Intermediate-element abundances in galaxy clusters. ApJ 620:680-696

Belsole E, Sauvageot JL, Pratt GW, Bourdin H (2005) An XMM-Newton observation of A3921: an off-axis merger. A\&A 430:385-397. doi:10.1051/0004-6361:20041275. arXiv:astro-ph/0409544

Birkinshaw M (1999) The Sunyaev-Zel'dovich effect. Phys Rep 310:97-195. doi:10.1016/ S0370-1573(98)00080-5. arXiv:astro-ph/9808050

Böhringer H (1998) Equilibrium ionization and non-equilibrium ionization plasma models. In: Breitschwerdt D, Freiberg MJ, Trümper J (eds) The local bubble and beyond. Springer, Heidelberg, p 341

Böhringer H, Hensler G (1989) Metallicity-dependence of radiative cooling in optically thin, hot plasmas. A\&A 215:147-149

Böhringer H, Nulsen PEJ, Braun R, Fabian AC (1995) The interaction of the radio halo of M87 with the cooling intracluster medium of the Virgo cluster. MNRAS 274:L67-L71

Böhringer H, Belsole E, Kennea J, Matsushita K, Molendi S, Worrall DM, Mushotzky RF, Ehle M, Guainazzi M, Sakelliou I, Stewart G, Vestrand WT, Dos Santos S (2001) XMM-Newton observations of M 87 and its X-ray halo. A\&A 365:L181-L187. doi:10.1051/0004-6361:20000092

Böhringer H, Matsushita K, Churazov E, Ikebe Y, Chen Y (2002) The new emerging model for the structure of cooling cores in clusters of galaxies. A\&A 382:804-820. doi:10.1051/0004-6361:20011708

Böhringer H, Matsushita K, Churazov E, Finoguenov A, Ikebe Y (2004) Implications of the central metal abundance peak in cooling core clusters of galaxies. A\&A 416:L21-L25. doi:10.1051/0004-6361: 20040047. arXiv:astro-ph/0402216

Borgani S, Rosati P, Tozzi P, Stanford SA, Eisenhardt PR, Lidman C, Holden B, Della Ceca R, Norman C, Squires G (2001) Measuring $\Omega_{m}$ with the ROSAT deep cluster survey. ApJ 561:13-21. doi:10.1086/ 323214. arXiv:astro-ph/0106428

Borgani S, Murante G, Springel V, Diaferio A, Dolag K, Moscardini L, Tormen G, Tornatore L, Tozzi P (2004) X-ray properties of galaxy clusters and groups from a cosmological hydrodynamical simulation. MNRAS 348:1078-1096. doi:10.1111/j.1365-2966.2004.07431.x. arXiv:astro-ph/0310794

Borgani S, Finoguenov A, Kay ST, Ponman TJ, Springel V, Tozzi P, Voit GM (2005) Entropy amplification from energy feedback in simulated galaxy groups and clusters. MNRAS 361:233-243. doi:10.1111/ j.1365-2966.2005.09158.x. arXiv:astro-ph/0504265 
Bourdin H, Sauvageot JL, Slezak E, Bijaoui A, Teyssier R (2004) Temperature map computation for X-ray clusters of galaxies. A\&A 414:429-443. doi:10.1051/0004-6361:20031662

Braglia F, Pierini D, Böhringer H (2007) Flaming, bright galaxies along the filaments of A 2744. A\&A 470:425-429. doi:10.1051/0004-6361:20077257. arXiv:0705.0273

Brickhouse NS, Raymond JC, Smith BW (1995) New model of iron spectra in the extreme ultraviolet and application to SERTS and EUV observations: A solar active region and capella. ApJS 97:551-570. doi:10.1086/192150

Briel UG, Henry JP (1994) An X-ray temperature map of the merging galaxy cluster A:2256. Nature 372:439. doi:10.1038/372439a0

Briel UG, Henry JP, Boehringer H (1992) Observation of the Coma cluster of galaxies with ROSAT during the all-sky survey. A\&A 259:L31-L34

Buote DA (2000a) Iron gradients in cooling flow galaxies and groups. ApJ 539:172-186. doi:10.1086/ 309224. arXiv:astro-ph/0001329

Buote DA (2000b) X-ray evidence for multiphase hot gas with nearly solar Fe abundances in the brightest groups of galaxies. MNRAS 311:176-200

Buote DA (2002) An XMM-Newton observation of NGC 1399 reveals two phases of hot gas and supersolar abundances in the central regions. ApJ 574:L135-L138. doi:10.1086/342532. arXiv:astro-ph/0206422

Buote DA, Canizares CR (1994) Geometrical evidence for dark matter: X-ray constraints on the mass of the elliptical galaxy NGC 720. ApJ 427:86-111. doi:10.1086/174123. arXiv:astro-ph/9311073

Buote DA, Fabian AC (1998) X-ray spectral analysis of elliptical galaxies from ASCA: the Fe abundance in a multiphase medium. MNRAS 296:977-994. arXiv:astro-ph/9707117

Buote DA, Lewis AD, Brighenti F, Mathews WG (2003) XMM-Newton and Chandra observations of the galaxy group NGC 5044. II. Metal abundances and supernova fraction. ApJ 595:151-166. doi:10. 1086/377256. arXiv:astro-ph/0303054

Buote DA, Gastaldello F, Humphrey PJ, Zappacosta L, Bullock JS, Brighenti F, Mathews WG (2007) The X-ray concentration-virial mass relation. ApJ 664:123-134. doi:10.1086/518684. arXiv:astro$\mathrm{ph} / 0610135$

Buote DA, Zappacosta L, Fang T, Humphrey PJ, Gastaldello F, Tagliaferri G (2009) X-ray absorption by WHIM in the sculptor wall. ApJ 695:1351. ArXiv:0901.3802

Bykov AM, Uvarov YA (1999) Electron kinetics in collisionless shock waves. JETP 88:465

Bykov AM, Dolag K, Durret F (2008) Cosmological shock waves. Space Sci Rev 134:119-140. doi:10. 1007/s11214-008-9312-9. arXiv:0801.0995

Canizares CR, Clark GW, Markert TH, Berg C, Smedira M, Bardas D, Schnopper H, Kalata K (1979) Highresolution X-ray spectroscopy of M87 with the Einstein observatory-the detection of an O VIII emission line. ApJ 234:L33-L37. doi:10.1086/183104

Canizares CR, Clark GW, Jernigan JG, Markert TH (1982) X-ray spectroscopy of the galaxy M87-radiative accretion of the hot plasma halo. ApJ 262:33-43. doi:10.1086/160393

Cappellari M, Copin Y (2003) Adaptive spatial binning of integral-field spectroscopic data using Voronoi tessellations. MNRAS 342:345-354. doi:10.1046/j.1365-8711.2003.06541.x. arXiv:astro-ph/0302262

Cavagnolo KW, Donahue M, Voit GM, Sun M (2009) Intracluster medium entropy profiles for a chandra archival sample of galaxy clusters. ApJS 182:12-32. doi:10.1088/0067-0049/182/1/12. arXiv: 0902.1802

Chiappini C, Matteucci F, Meynet G (2003) Stellar yields with rotation and their effect on chemical evolution models. A\&A 410:257-267. doi:10.1051/0004-6361:20031192

Churazov E, Brüggen M, Kaiser CR, Böhringer H, Forman W (2001) Evolution of buoyant bubbles in M87. ApJ 554:261-273. doi:10.1086/321357

Churazov E, Forman W, Jones C, Sunyaev R, Böhringer H (2004) XMM-Newton observations of the Perseus cluster-II. Evidence for gas motions in the core. MNRAS 347:29-35. doi:10.1111/j.1365-2966. 2004.07201.x. arXiv:astro-ph/0309427

Conselice CJ, Gallagher JS III, Wyse RFG (2001) On the nature of the NGC 1275 system. AJ 122:22812300. doi:10.1086/323534. arXiv:astro-ph/0108019

Cox DP (1972) Theoretical structure and spectrum of a shock wave in the interstellar medium: the cygnus loop. ApJ 178:143-158. doi:10.1086/151774

Cox DP, Daltabuit E (1971) Radiative cooling of a low-density plasma. ApJ 167:113. doi:10.1086/151009

Cox DP, Tucker WH (1969) Ionization equilibrium and radiative cooling of a low-density plasma. ApJ 157:1157. doi:10.1086/150144 
David LP, Jones C, Forman W (1996) ROSAT PSPC observations of cool rich clusters. ApJ 473:692. doi:10. $1086 / 178182$

David LP, Nulsen PEJ, McNamara BR, Forman W, Jones C, Ponman T, Robertson B, Wise M (2001) A high-resolution study of the Hydra A cluster with chandra: comparison of the core mass distribution with theoretical predictions and evidence for feedback in the cooling flow. ApJ 557:546-559. doi:10. 1086/322250. arXiv:astro-ph/0010224

De Grandi S, Molendi S (2001) Metallicity gradients in X-ray clusters of galaxies. ApJ 551:153-159. doi:10.1086/320098. arXiv:astro-ph/0012232

De Grandi S, Molendi S (2002) Temperature profiles of nearby clusters of galaxies. ApJ 567:163-177

De Grandi S, Ettori S, Longhetti M, Molendi S (2004) On the iron content in rich nearby clusters of galaxies. A\&A 419:7-18. doi:10.1051/0004-6361:20034228. arXiv:astro-ph/0310828

de Plaa J, Werner N, Bykov AM, Kaastra JS, Méndez M, Vink J, Bleeker JAM, Bonamente M, Peterson JR (2006) Chemical evolution in Sérsic 159-03 observed with XMM-Newton. A\&A 452:397-412. doi:10. 1051/0004-6361:20053864. arXiv:astro-ph/0602582

de Plaa J, Werner N, Bleeker JAM, Vink J, Kaastra JS, Méndez M (2007) Constraining supernova models using the hot gas in clusters of galaxies. A\&A 465:345-355. doi:10.1051/0004-6361:20066382. arXiv:astro-ph/0701553

Diehl S, Statler TS (2006) Adaptive binning of X-ray data with weighted Voronoi tessellations. MNRAS 368:497-510. doi:10.1111/j.1365-2966.2006.10125.x. arXiv:astro-ph/0512074

Domainko W, Mair M, Kapferer W, van Kampen E, Kronberger T, Schindler S, Kimeswenger S, Ruffert M, Mangete OE (2006) Enrichment of the ICM of galaxy clusters due to ram-pressure stripping. A\&A 452:795-802. doi:10.1051/0004-6361:20053921. arXiv:astro-ph/0507605

Donahue M, Voit GM, Scharf CA, Gioia IM, Mullis CR, Hughes JP, Stocke JT (1999) The second most distant cluster of galaxies in the extended medium sensitivity survey. ApJ 527:525-534. doi:10.1086/ 308101

Dressler A (1980) Galaxy morphology in rich clusters - implications for the formation and evolution of galaxies. ApJ 236:351-365. doi:10.1086/157753

Dressler A, Oemler AJ, Couch WJ, Smail I, Ellis RS, Barger A, Butcher H, Poggianti BM, Sharples RM (1997) Evolution since $Z=0.5$ of the morphology-density relation for clusters of galaxies. ApJ 490:577. doi:10.1086/304890. arXiv:astro-ph/9707232

Dupke RA, Arnaud KA (2001) Central elemental abundance ratios in the perseus cluster: resonant scattering or SN IA enrichment? ApJ 548:141-149. doi:10.1086/318694. arXiv:astro-ph/0010054

Dupke RA, White RE III (2000) Constraints on type IA supernova models from X-ray spectra of galaxy clusters. ApJ 528:139-144. doi:10.1086/308181. arXiv:astro-ph/9907343

Durret F, Lima Neto GB, Forman W (2005) An XMM-Newton view of the cluster of galaxies Abell 85. A\&A 432:809-821. doi:10.1051/0004-6361:20041666. arXiv:astro-ph/0411527

Durret F, Kaastra JS, Nevalainen J, Ohashi T, Werner N (2008) Soft X-ray and extreme ultraviolet excess emission from clusters of galaxies. Space Sci Rev 134:51-70. doi:10.1007/s11214-008-9313-8. arXiv:0801.0977

Eckert D, Neronov A, Courvoisier TJL, Produit N (2007) South-West extension of the hard X-ray emission from the Coma cluster. A\&A 470:835-841. doi:10.1051/0004-6361:20077268. arXiv:0705.2722

Evrard AE, Metzler CA, Navarro JF (1996) Mass estimates of X-ray clusters. ApJ 469:494

Ettori S, Fabian AC (2006) Effects of sedimented helium on the X-ray properties of galaxy clusters. MNRAS 369:L42-L46. doi:10.1111/j.1745-3933.2006.00170.x. arXiv:astro-ph/0603383

Fabian AC (1994) Cooling flows in clusters of galaxies. ARA\&A 32:277-318

Fabian AC, Nulsen PEJ (1977) Subsonic accretion of cooling gas in clusters of galaxies. MNRAS 180:479_ 484

Fabian AC, Pringle JE (1977) On the spatial distribution of heavy elements in X-ray emitting clusters of galaxies. MNRAS 181:5P-7P

Fabian AC, Sanders JS, Taylor GB, Allen SW (2005) A deep Chandra observation of the Centaurus cluster: bubbles, filaments and edges. MNRAS 360:L20-L24. doi:10.1111/j.1745-3933.2005.00037.x. arXiv:astro-ph/0503154

Fabian AC, Sanders JS, Taylor GB, Allen SW, Crawford CS, Johnstone RM, Iwasawa K (2006) A very deep Chandra observation of the Perseus cluster: shocks, ripples and conduction. MNRAS 366:417-428. doi:10.1111/j.1365-2966.2005.09896.x. arXiv:astro-ph/0510476

Feretti L, Giovannini G (1998) Diffuse radio emission in the Coma cluster. In: Mazure A, Casoli F, Durret F, Gerbal D (eds) Untangling coma berenices: a new vision of an old cluster, p 123 
Feretti L, Giovannini G (2008) Galaxy clusters in the radio: relativistic plasma and ICM/radio galaxy interaction processes. LNP 740:143 arXiv:astro-ph/0703494

Feretti L, Gioia IM, Giovannini G (eds) (2002) Merging processes in galaxy clusters. Astrophysics and Space Science Library, vol 272

Finoguenov A, David LP, Ponman TJ (2000) An ASCA study of the heavy-element distribution in clusters of galaxies. ApJ 544:188-203. doi:10.1086/317173. arXiv:astro-ph/9908150

Finoguenov A, Matsushita K, Böhringer H, Ikebe Y, Arnaud M (2002) X-ray evidence for spectroscopic diversity of type Ia supernovae: XMM observation of the elemental abundance pattern in M87. A\&A 381:21-31. doi:10.1051/0004-6361:20011477

Finoguenov A, Briel UG, Henry JP (2003) XMM-Newton discovery of an X-ray filament in Coma. A\&A 410:777-784

Finoguenov A, Böhringer H, Zhang Y-Y (2005) XMM-Newton study of the two-dimensional structure of the REFLEX-DXL galaxy clusters. A\&A 442:827-839

Forman W, Nulsen P, Heinz S, Owen F, Eilek J, Vikhlinin A, Markevitch M, Kraft R, Churazov E, Jones C (2005) Reflections of active galactic nucleus outbursts in the gaseous atmosphere of M87. ApJ 635:894-906. doi:10.1086/429746. arXiv:astro-ph/0312576

Forman W, Jones C, Churazov E, Markevitch M, Nulsen P, Vikhlinin A, Begelman M, Böhringer H, Eilek J, Heinz S, Kraft R, Owen F, Pahre M (2007) Filaments, bubbles, and weak shocks in the gaseous atmosphere of M87. ApJ 665:1057-1066. doi:10.1086/519480. arXiv:astro-ph/0604583

Fukazawa Y, Ohashi T, Fabian AC, Canizares CR, Ikebe Y, Makishima K, Mushotzky RF, Yamashita K (1994) Metal concentration and X-ray cool spectral component in the central region of the Centaurus cluster of galaxies. PASJ 46:L55-L58

Fukazawa Y, Makishima K, Tamura T, Ezawa H, Xu H, Ikebe Y, Kikuchi K, Ohashi T (1998) ASCA measurements of silicon and iron abundances in the intracluster medium. PASJ 50:187-193. arXiv:astro$\mathrm{ph} / 9802126$

Fusco-Femiano R, dal Fiume D, Feretti L, Giovannini G, Grandi P, Matt G, Molendi S, Santangelo A (1999) Hard X-ray radiation in the coma cluster spectrum. ApJ 513:L21-L24. doi:10.1086/311902. arXiv:astro-ph/9901018

Gaetz TJ, Salpeter EE (1983) Line radiation from a hot, optically thin plasma-collision strengths and emissivities. ApJS 52:155-168. doi:10.1086/190862

Gallagher JS, Garnavich PM, Berlind P, Challis P, Jha S, Kirshner RP (2005) Chemistry and star formation in the host galaxies of type Ia supernovae. ApJ 634:210-226. doi:10.1086/491664. arXiv:astro$\mathrm{ph} / 0508180$

Gao L, Navarro JF, Cole S, Frenk CS, White SDM, Springel V, Jenkins A, Neto AF (2008) The redshift dependence of the structure of massive $\Lambda$ cold dark matter haloes. MNRAS 387:536-544. doi:10. 1111/j.1365-2966.2008.13277.x. arXiv:0711.0746

Gastaldello F, Molendi S (2004) Ni abundance in the core of the perseus cluster: an answer to the significance of resonant scattering. ApJ 600:670-680. doi:10.1086/379970. arXiv:astro-ph/0309582

George MR, Fabian AC, Sanders JS, Young AJ, Russell HR (2009) X-ray observations of the galaxy cluster PKS0745-191: to the virial radius, and beyond. MNRAS 395:657-666. doi:10.1111/j.1365-2966. 2009.14547.x. arXiv:0807.1130

Gilfanov MR, Syunyaev RA (1984) Intracluster gravitational separation of deuterium and helium in rich galaxy clusters. Sov Astron Lett 10:137

Gilfanov MR, Sunyaev RA, Churazov EM (1987) Radial brightness profiles of resonance X-ray lines in galaxy clusters. Sov Astron Lett 13:3-7

Grevesse N, Sauval AJ (1998) Standard solar composition. Space Sci Rev 85:161-174

Gronenschild EHBM, Mewe R (1978) Calculated X-radiation from optically thin plasmas. III-abundance effects on continuum emission. A\&AS 32:283-305

Gustafsson B, Karlsson T, Olsson E, Edvardsson B, Ryde N (1999) The origin of carbon, investigated by spectral analysis of solar-type stars in the Galactic Disk. A\&A 342:426-439

Hamuy M, Phillips MM, Suntzeff NB, Schommer RA, Maza J, Smith RC, Lira P, Aviles R (1996) The morphology of type IA supernovae light curves. AJ 112:2438. doi:10.1086/118193. arXiv:astro$\mathrm{ph} / 9609063$

Hatch NA, Crawford CS, Fabian AC, Johnstone RM (2005) Detections of molecular hydrogen in the outer filaments of NGC1275. MNRAS 358:765-773. doi:10.1111/j.1365-2966.2005.08787.x. arXiv:astro$\mathrm{ph} / 0411446$

Hattori M, Kneib J, Makino N (1999) Gravitational lensing in clusters of galaxies. Prog Theor Phys Suppl 133:1-51. doi:10.1143/PTPS.133.1. arXiv:astro-ph/9905009 
Henry JP (2004) X-ray temperatures for the extended medium-sensitivity survey high-redshift cluster sample: constraints on cosmology and the dark energy equation of state. ApJ 609:603-616. doi:10.1086/ 421336. arXiv:astro-ph/0404142

Henry JP, Briel UG (1995) An X-ray temperature map of Abell 754: a major merger. ApJ 443:L9-L12. doi:10.1086/187823

Henry JP, Evrard AE, Hoekstra H, Babul A, Mahdavi A (2009) The X-ray cluster normalization of the matter power spectrum. ApJ 691:1307-1321. doi:10.1088/0004-637X/691/2/1307. arXiv:0809.3832

Ikebe Y, Makishima K, Ezawa H, Fukazawa Y, Hirayama M, Honda H, Ishisaki Y, Kikuchi K, Kubo H, Murakami T, Ohashi T, Takahashi T, Yamashita K (1997) Structure of the X-ray-emitting gas in the hydra a cluster of galaxies. ApJ 481:660. doi:10.1086/304095. arXiv:astro-ph/9701133

Inoue S, Aharonian FA, Sugiyama N (2005) Hard X-ray and gamma-ray emission induced by ultrahigh-energy protons in cluster accretion shocks. ApJ 628:L9-L12. doi:10.1086/432602. arXiv:astro$\mathrm{ph} / 0505398$

Ivanov VD, Hamuy M, Pinto PA (2000) On the relation between peak luminosity and parent population of type IA supernovae: a new tool for probing the ages of distant galaxies. ApJ 542:588-596. doi:10. 1086/317060. arXiv:astro-ph/0006047

Iwamoto K, Brachwitz F, Nomoto K, Kishimoto N, Umeda H, Hix WR, Thielemann F (1999) Nucleosynthesis in Chandrasekhar mass models for type IA supernovae and constraints on progenitor systems and burning-front propagation. ApJS 125:439-462

Kaastra JS (1992) An X-ray spectral code for optically thin plasmas (Internal SRON-Leiden Report, Updated Version 2.0)

Kaastra JS, Mewe R (1994) Journal of the HEASARC at NASA GSFC, vol 3, p 16

Kaastra JS, Mewe R, Nieuwenhuijzen H (1996) Spex: a new code for spectral analysis of X and UV spectra. In: Yamashita K, Watanabe T (eds) UV and X-ray spectroscopy of astrophysical and laboratory plasmas. Universal Academy Press, Tokyo, p 411

Kaastra JS, Tamura T, Peterson JR, Bleeker J, Ferrigno C, Kahn SM, Paerels FBS, Piffaretti R, BranduardiRaymont G, Böhringer H (2004) Spatially resolved X-ray spectroscopy of cooling clusters of galaxies. A\&A 413:415-439

Kaastra JS, Bykov A, Werner N (2009) Non-Maxwellian electron distributions in clusters of galaxies. A\&A. arXiv 0905.4802 (in press)

Kato T (1976) Radiation from a hot, thin plasma from 1 to 250 A. ApJS 30:397-449. doi:10.1086/190367

Kolmogorov A (1941) The local structure of turbulence in incompressible viscous fluid for very large Reynolds' numbers. Akademiia Nauk SSSR Doklady 30:301-305

Kotov O, Vikhlinin A (2006) Chandra sample of galaxy clusters at $z=0.4-0.55$ : evolution in the masstemperature relation. ApJ 641:752-755. doi:10.1086/500553. arXiv:astro-ph/0511044

Kravtsov AV, Vikhlinin A, Nagai D (2006) A new robust low-scatter X-ray mass indicator for clusters of galaxies. ApJ 650:128-136. doi:10.1086/506319. arXiv:astro-ph/0603205

Landini M, Fossi BCM (1991) Ion equilibrium for minor components in a thin plasma. A\&AS 91:183-196

Landini M, Monsignori Fossi BC (1970) Solar radiation from 1 to 100 A. A\&A 6:468-H

Landini M, Monsignori Fossi BC (1990) The X-UV spectrum of thin plasmas. A\&AS 82:229-260

Liedahl DA, Osterheld AL, Goldstein WH (1995) New calculations of Fe L-shell X-ray spectra in hightemperature plasmas. ApJ 438:L115-L118. doi:10.1086/187729

Lin YT, Mohr JJ, Stanford SA (2003) Near-infrared properties of galaxy clusters: luminosity as a binding mass predictor and the state of cluster baryons. ApJ 591:749-763. doi:10.1086/375513. arXiv:astro$\mathrm{ph} / 0304033$

Lloyd-Davies EJ, Ponman TJ, Cannon DB (2000) The entropy and energy of intergalactic gas in galaxy clusters. MNRAS 315:689-702. doi:10.1046/j.1365-8711.2000.03380.x. arXiv:astro-ph/0002082

Lodders K (2003) Solar system abundances and condensation temperatures of the elements. ApJ 591:12201247

Lutovinov AA, Vikhlinin A, Churazov EM, Revnivtsev MG, Sunyaev RA (2008) X-ray observations of the Coma Cluster in a broad energy band with INTEGRAL, RXTE, and ROSAT observatories. ApJ 687:968. ArXiv 0802.3742

Mahdavi A, Hoekstra H, Babul A, Henry JP (2008) Evidence for non-hydrostatic gas from the cluster X-ray to lensing mass ratio. MNRAS 384:1567-1574. doi:10.1111/j.1365-2966.2007.12796.x. arXiv:0710.4132

Makishima K (1999) Energy non-equipartition processes in the Universe. Astron Nachr 320:163

Mannucci F, Della Valle M, Panagia N (2006) Two populations of progenitors for Type Ia supernovae? MNRAS 370:773-783. doi:10.1111/j.1365-2966.2006.10501.x. arXiv:astro-ph/0510315 
Markevitch M (2006) Chandra observation of the most interesting cluster in the Universe. In: Wilson A (ed) The X-ray Universe 2005, ESA Special Publication, vol 604, pp 723-727

Markevitch M, Vikhlinin A (2007) Shocks and cold fronts in galaxy clusters. Phys Rep 443:1-53. doi:10. 1016/j.physrep.2007.01.001. arXiv:astro-ph/0701821

Markevitch M, Forman WR, Sarazin CL, Vikhlinin A (1998) The temperature structure of 30 nearby clusters observed with ASCA: similarity of temperature profiles. ApJ 503:77. doi:10.1086/305976. arXiv:astro-ph/9711289

Markevitch M, Gonzalez AH, David L, Vikhlinin A, Murray S, Forman W, Jones C, Tucker W (2002) A textbook example of a bow shock in the merging galaxy cluster 1E 0657-56. ApJ 567:L27-L31. doi:10.1086/339619. arXiv:astro-ph/0110468

Markevitch M, Govoni F, Brunetti G, Jerius D (2005) Bow shock and radio halo in the merging cluster A520. ApJ 627:733-738. doi:10.1086/430695. arXiv:astro-ph/0412451

Masai K (1984) X-ray emission spectra from ionizing plasmas. Astrophys Space Sci 98:367-395. doi:10. 1007/BF00651415

Masai K, Kato T (1987) Electron density diagnostics by Fe XXII line intensity ratio. Phys Lett A 123:405409. doi:10.1016/0375-9601(87)90042-9

Mastropietro C, Burkert A (2008) Simulating the bullet cluster. MNRAS 389:967-988. doi:10.1111/j. 1365-2966.2008.13626.x. arXiv:0711.0967

Mathiesen BF, Evrard AE (2001) Four measures of the intracluster medium temperature and their relation to a cluster's dynamical state. ApJ 546:100-116. doi:10.1086/318249. arXiv:astro-ph/0004309

Matsushita K, Belsole E, Finoguenov A, Böhringer H (2002) XMM-Newton observation of M 87. I. Single-phase temperature structure of intracluster medium. A\&A 386:77-96. doi:10.1051/0004-6361: 20020087

Matsushita K, Finoguenov A, Böhringer H (2003) XMM observation of M 87. II. Abundance structure of the interstellar and intergalactic medium. A\&A 401:443-461. doi:10.1051/0004-6361:20021791

Matsushita K, Böhringer H, Takahashi I, Ikebe Y (2007a) The abundance pattern of O, Mg, Si, and Fe in the intracluster medium of the Centaurus cluster observed with XMM-Newton. A\&A 462:953-963. doi:10.1051/0004-6361:20041577. arXiv:astro-ph/0610293

Matsushita K, Fukazawa Y, Hughes JP, Kitaguchi T, Makishima K, Nakazawa K, Ohashi T, Ota N, Tamura T, Tozuka M, Tsuru TG, Urata Y, Yamasaki NY (2007b) Suzaku observation of the metallicity distribution in the intracluster medium of the fornax cluster. PASJ 59:327-338. arXiv:astro-ph/0609065

Maughan BJ, Jones C, Forman W, Van Speybroeck L (2008) Images, structural properties, and metal abundances of galaxy clusters observed with Chandra ACIS-I at 0.1 $<z<1.3$. ApJS 174:117-135. doi:10. 1086/521225. arXiv:astro-ph/0703156

Mazzali PA, Röpke FK, Benetti S, Hillebrandt W (2007) A common explosion mechanism for type Ia supernovae. Science 315:825-828. doi:10.1126/science.1136259. arXiv:astro-ph/0702351

Mazzotta P, Mazzitelli G, Colafrancesco S, Vittorio N (1998) Ionization balance for optically thin plasmas: rate coefficients for all atoms and ions of the elements H to NI. A\&AS 133:403-409. doi:10.1051/ aas:1998330. arXiv:astro-ph/9806391

Mazzotta P, Rasia E, Moscardini L, Tormen G (2004) Comparing the temperatures of galaxy clusters from hydrodynamical N-body simulations to Chandra and XMM-Newton observations. MNRAS 354: 10-24. doi:10.1111/j.1365-2966.2004.08167.x. arXiv:astro-ph/0409618

McCarthy IG, Babul A, Bower RG, Balogh ML (2008) Towards a holistic view of the heating and cooling of the intracluster medium. MNRAS 386:1309-1331. doi:10.1111/j.1365-2966.2008.13141.x. arXiv:0706.2768

Mei S, Holden BP, Blakeslee JP, Rosati P, Postman M, Jee MJ, Rettura A, Sirianni M, Demarco R, Ford HC, Franx M, Homeier N, Illingworth GD (2006) Evolution of the color-magnitude relation in high-redshift clusters: early-type galaxies in the Lynx supercluster at $z \sim 1.26$. ApJ 644:759-768. doi:10. 1086/503826. arXiv:astro-ph/0604082

Mewe R (1990) Ionization of hot plasmas. In: NATO ASIC Proc. 300: physical processes in hot cosmic plasmas, pp 39-65

Mewe R (1991) X-ray spectroscopy of stellar coronae. A\&AR 3:127-168. doi:10.1007/BF00873539

Mewe R, Gronenschild EHBM (1981) Calculated X-radiation from optically thin plasmas. IV-atomic data and rate coefficients for spectra in the range 1-270 A. A\&AS 45:11-52

Mewe R, Gronenschild EHBM, van den Oord GHJ (1985) Calculated X-radiation from optically thin plasmas. V. A\&AS 62:197-254 
Mewe R, Lemen JR, van den Oord GHJ (1986) Calculated X-radiation from optically thin plasmas. VI-improved calculations for continuum emission and approximation formulae for nonrelativistic average Gaunt factors. A\&AS 65:511-536

Mewe R, Kaastra JS, Liedahl DA (1995) Update of MEKA: MEKAL. Legacy 6:16

Meynet G, Maeder A (2002) The origin of primary nitrogen in galaxies. A\&A 381:L25-L28. doi:10.1051/ 0004-6361:20011554

Million ET, Allen SW (2008) Chandra measurements of complex X-ray emission from massive, merging, radio-halo clusters. MNRAS. ArXiv:0811.0834 (in press)

Mitchell RJ, Culhane JL, Davison PJN, Ives JC (1976) Ariel 5 observations of the X-ray spectrum of the Perseus Cluster. MNRAS 175:29P-34P

Molendi S, Gastaldello F (2001) On the metal abundance in the core of M 87. A\&A 375:L14-L17

Moore B, Ghigna S, Governato F, Lake G, Quinn T, Stadel J, Tozzi P (1999) Dark matter substructure within galactic halos. ApJ 524:L19-L22. doi:10.1086/312287. arXiv:astro-ph/9907411

Mushotzky RF (1984) X-ray emission from clusters of galaxies. Phys Scr Vol T 7:157-162

Mushotzky RF, Loewenstein M (1997) Lack of evolution in the iron abundance in clusters of galaxies and implications for the global star formation rate at high redshift. ApJ 481:L63. doi:10.1086/310651. arXiv:astro-ph/9702149

Mushotzky RF, Serlemitsos PJ, Boldt EA, Holt SS, Smith BW (1978) OSO 8 X-ray spectra of clusters of galaxies. I-observations of twenty clusters: physical correlations. ApJ 225:21-39. doi:10.1086/ 156465

Mushotzky R, Loewenstein M, Arnaud KA, Tamura T, Fukazawa Y, Matsushita K, Kikuchi K, Hatsukade I (1996) Measurement of the elemental abundances in four rich clusters of galaxies. I. Observations. ApJ 466:686. doi:10.1086/177541

Nakazawa K, Sarazin CL, Kawaharada M, Kitaguchi T, Okuyama S, Makishima K, Kawano N, Fukazawa Y, Inoue S, Takizawa M, Wik DR, Finoguenov A, Clarke TE (2009) Hard X-ray properties of the merging cluster Abell 3667 as observed with Suzaku. PASJ 61:339. arXiv:0812.1438

Navarro JF, Frenk CS, White SDM (1995) Simulations of X-ray clusters. MNRAS 275:720-740. arXiv:astro-ph/9408069

Navarro JF, Frenk CS, White SDM (1997) A universal density profile from hierarchical clustering. ApJ 490:493-508

Nomoto K, Tominaga N, Umeda H, Kobayashi C, Maeda K (2006) Nucleosynthesis yields of core-collapse supernovae and hypernovae, and galactic chemical evolution. Nucl Phys A 777:424-458. doi:10.1016/ j.nuclphysa.2006.05.008. arXiv:astro-ph/0605725

Nulsen PEJ, David LP, McNamara BR, Jones C, Forman WR, Wise M (2002) Interaction of radio lobes with the hot intracluster medium: driving convective outflow in Hydra A. ApJ 568:163-173. doi:10. 1086/338494. arXiv:astro-ph/0110523

Nulsen PEJ, McNamara BR, Wise MW, David LP (2005) The cluster-scale AGN outburst in Hydra A. ApJ 628:629-636. doi:10.1086/430845. arXiv:astro-ph/0408315

Oboukhov A (1941) On the distribution of energy in the spectrum of turbulent flow. Dokl Akad Nauk SSSR $32: 22$

Owen FN, Eilek JA, Kassim NE (2000) M87 at 90 centimeters: a different picture. ApJ 543:611-619. doi:10.1086/317151

Paerels F, Kaastra J, Ohashi T, Richter P, Bykov A, Nevalainen J (2008) Future instrumentation for the study of the warm-hot intergalactic medium. Space Sci Rev 134:405-418. doi:10.1007/s11214-008-9323-6. arXiv:0801.1064

Peng F, Nagai D (2009) Effect of helium sedimentation on X-ray measurements of galaxy clusters. ApJ 693:839-846. doi:10.1088/0004-637X/693/1/839. arXiv:0808.3769

Peres CB, Fabian AC, Edge AC, Allen SW, Johnstone RM, White DA (1998) A ROSAT study of the cores of clusters of galaxies-I. cooling flows in an X-ray flux-limited sample. MNRAS 298:416-432. arXiv:astro-ph/9805122

Peterson JR, Paerels FBS, Kaastra JS, Arnaud M, Reiprich TH, Fabian AC, Mushotzky RF, Jernigan JG, Sakelliou I (2001) X-ray imaging-spectroscopy of Abell 1835. A\&A 365:L104-L109

Peterson JR, Kahn SM, Paerels FBS, Kaastra JS, Tamura T, Bleeker JAM, Ferrigno C, Jernigan JG (2003) High-resolution X-ray spectroscopic constraints on cooling-flow models for clusters of galaxies. ApJ 590:207-224

Petrosian V (2001) On the nonthermal emission and acceleration of electrons in coma and other clusters of galaxies. ApJ 557:560-572. doi:10.1086/321557. arXiv:astro-ph/0101145 
Petrosian V, Bykov AM (2008) Particle acceleration mechanisms. Space Sci Rev 134:207-227. doi:10. 1007/s11214-008-9315-6. arXiv:0801.0923

Poggianti BM, Smail I, Dressler A, Couch WJ, Barger AJ, Butcher H, Ellis RS, Oemler AJ (1999) The star formation histories of galaxies in distant clusters. ApJ 518:576-593. doi:10.1086/307322. arXiv:astro-ph/9901264

Pointecouteau E, Arnaud M, Pratt GW (2005) The structural and scaling properties of nearby galaxy clusters. I. The universal mass profile. A\&A 435:1-7. doi:10.1051/0004-6361:20042569. arXiv:astro$\mathrm{ph} / 0501635$

Ponman TJ, Cannon DB, Navarro JF (1999) The thermal imprint of galaxy formation on X-ray clusters. Nature 397:135-137. doi:10.1038/16410. arXiv:astro-ph/9810359

Porquet D, Arnaud M, Decourchelle A (2001) Impacts of a power-law non-thermal electron tail on the ionization and recombination rates. A\&A 373:1110-1124. doi:10.1051/0004-6361:20010667. arXiv:astro$\mathrm{ph} / 0105376$

Pratt GW (2006) X-ray observations of the mass and entropy distributions in nearby galaxy clusters. arXiv:astro-ph/0607199, arXiv:astro-ph/0607199

Pratt GW, Arnaud M (2002) The mass profile of A1413 observed with XMM-Newton: implications for the M-T relation. A\&A 394:375-393

Pratt GW, Arnaud M, Pointecouteau E (2006) Structure and scaling of the entropy in nearby galaxy clusters. A\&A 446:429-438. doi:10.1051/0004-6361:20054025. arXiv:astro-ph/0508234

Pratt GW, Böhringer H, Croston JH, Arnaud M, Borgani S, Finoguenov A, Temple RF (2007) Temperature profiles of a representative sample of nearby X-ray galaxy clusters. A\&A 461:71-80. doi:10.1051/ 0004-6361:20065676. arXiv:astro-ph/0609480

Pratt GW, Croston JH, Arnaud M, Boehringer H (2009) Galaxy cluster X-ray luminosity scaling relations from a representative local sample (REXCESS). A\&A 498:361. arXiv:08093784 0809.3784

Rasia E, Mazzotta P, Borgani S, Moscardini L, Dolag K, Tormen G, Diaferio A, Murante G (2005) Mismatch between X-ray and emission-weighted temperatures in galaxy clusters: cosmological implications. ApJ 618:L1-L4. doi:10.1086/427554. arXiv:astro-ph/0409650

Rasia E, Ettori S, Moscardini L, Mazzotta P, Borgani S, Dolag K, Tormen G, Cheng LM, Diaferio A (2006) Systematics in the X-ray cluster mass estimators. MNRAS 369:2013-2024. doi:10.1111/j.1365-2966. 2006.10466.x. arXiv:astro-ph/0602434

Rasia E, Mazzotta P, Bourdin H, Borgani S, Tornatore L, Ettori S, Dolag K, Moscardini L (2008) X-MAS2: study systematics on the ICM metallicity measurements. ApJ 674:728-741. doi:10.1086/524345. arXiv:0707.2614

Raymond JC (1988) Radiation from Hot, thin plasmas. In: Pallavicini R (ed) NATO ASIC Proceedings, vol 249, Hot thin plasmas in astrophysics, p 3

Raymond JC, Brickhouse NS (1996) Atomic processes in astrophysics. Astrophys Space Sci 237:321-340. doi:10.1007/BF02424437

Raymond JC, Smith BW (1977) Soft X-ray spectrum of a hot plasma. ApJS 35:419-439. doi:10.1086/ 190486

Rebusco P, Churazov E, Böhringer H, Forman W (2005) Impact of stochastic gas motions on galaxy cluster abundance profiles. MNRAS 359:1041-1048. doi:10.1111/j.1365-2966.2005.08965.x. arXiv:astro$\mathrm{ph} / 0501141$

Rebusco P, Churazov E, Böhringer H, Forman W (2006) Effect of turbulent diffusion on iron abundance profiles. MNRAS 372:1840-1850. doi:10.1111/j.1365-2966.2006.10977.x. arXiv:astro-ph/0608491

Reiprich TH, Sarazin CL, Kempner JC, Tittley E (2004) XMM-newton observation of the merging galaxy cluster A1644. ApJ 608:179-188. doi:10.1086/386318. arXiv:astro-ph/0308282

Reiprich TH, Hudson DS, Zhang Y, Sato K, Ishisaki Y, Hoshino A, Ohashi T, Ota N, Fujita Y (2008) Suzaku measurement of Abell 2204's intracluster gas temperature profile out to $1800 \mathrm{kpc}$. A\&A 501:899. ArXiv e-prints 0806.2920

Renzini A (1997) Iron as a tracer in galaxy clusters and groups. ApJ 488:35. doi:10.1086/304696. arXiv:astro-ph/9706083

Rephaeli Y (1977) Spatial distribution of Compton-produced X-ray flux from rich and regular clusters of galaxies. ApJ 212:608-615

Rephaeli Y, Gruber D (2002) Results from a second Rossi X-ray timing explorer observation of the coma cluster. ApJ 579:587-591. doi:10.1086/342796. arXiv:astro-ph/0207443

Rephaeli Y, Nevalainen J, Ohashi T, Bykov AM (2008) Nonthermal phenomena in clusters of galaxies. Space Sci Rev 134:71-92. doi:10.1007/s11214-008-9314-7. arXiv:0801.0982 
Ricker PM, Sarazin CL (2001) Off-axis cluster mergers: effects of a strongly peaked dark matter profile. ApJ 561:621-644. doi:10.1086/323365. arXiv:astro-ph/0107210

Rizza E, Burns JO, Ledlow MJ, Owen FN, Voges W, Bliton M (1998) X-ray observations of distant Abell clusters. MNRAS 301:328-342

Sanders JS (2006) Contour binning: a new technique for spatially resolved X-ray spectroscopy applied to Cassiopeia A. MNRAS 371:829-842. doi:10.1111/j.1365-2966.2006.10716.x. arXiv:astro-ph/0606 528

Sanders JS, Fabian AC (2002) Spatially resolved X-ray spectroscopy of the core of the Centaurus cluster. MNRAS 331:273-283

Sanders JS, Fabian AC (2006) Enrichment in the Centaurus cluster of galaxies. MNRAS 371:1483-1496. doi:10.1111/j.1365-2966.2006.10779.x. arXiv:astro-ph/0607113

Sanders JS, Fabian AC (2007) A deeper X-ray study of the core of the Perseus galaxy cluster: the power of sound waves and the distribution of metals and cosmic rays. MNRAS 381:1381-1399. doi:10.1111/ j.1365-2966.2007.12347.x. arXiv:0705.2712

Sanders JS, Fabian AC, Allen SW, Schmidt RW (2004) Mapping small-scale temperature and abundance structures in the core of the Perseus cluster. MNRAS 349:952-972

Sanders JS, Fabian AC, Allen SW, Morris RG, Graham J, Johnstone RM (2008) Cool X-ray emitting gas in the core of the Centaurus cluster of galaxies. MNRAS 385:1186-1200. doi:10.1111/j.1365-2966. 2008.12952.x. arXiv:0711.2456

Sanderson AJR, Ponman TJ, Finoguenov A, Lloyd-Davies EJ, Markevitch M (2003) The Birmingham-CfA cluster scaling project-I. Gas fraction and the $M-T_{X}$ relation. MNRAS 340:989-1010. doi:10. 1046/j.1365-8711.2003.06401.x. arXiv:astro-ph/0301049

Sarazin CL (1986) X-ray emission from clusters of galaxies. Rev Mod Phys 58:1-115

Sato K, Yamasaki NY, Ishida M, Ishisaki Y, Ohashi T, Kawahara H, Kitaguchi T, Kawaharada M, Kokubun M, Makishima K, Ota N, Nakazawa K, Tamura T, Matsushita K, Kawano N, Fukazawa Y, Hughes JP (2007) X-ray study of temperature and abundance profiles of the cluster of galaxies Abell 1060 with Suzaku. PASJ 59:299-317. arXiv:astro-ph/0701328

Sato K, Matsushita K, Ishisaki Y, Sasaki S, Ohashi T, Yamasaki NY, Ishida M (2008) Suzaku observations of AWM 7 cluster of galaxies: temperatures, abundances, and bulk motions. PASJ 60:333. arXiv:0707.4342

Sato K, Matsushita K, Ishisaki Y, Yamasaki NY, Ishida M, Ohashi T (2009) Suzaku observation of group of galaxies NGC 507: temperature and metal distributions in the intra-cluster medium. PASJ 61:353-363

Schmutzler T, Tscharnuter WM (1993) Effective radiative cooling in optically thin plasmas. A\&A 273:318

Schuecker P, Böhringer H, Collins CA, Guzzo L (2003a) The REFLEX galaxy cluster survey. VII. Omega $a_{m}$ and sigma $_{8}$ from cluster abundance and large-scale clustering. A\&A 398:867-877. doi:10.1051/ 0004-6361:20021715. arXiv:astro-ph/0208251

Schuecker P, Caldwell RR, Böhringer H, Collins CA, Guzzo L, Weinberg NN (2003b) Observational constraints on general relativistic energy conditions, cosmic matter density and dark energy from X-ray clusters of galaxies and type-Ia supernovae. A\&A 402:53-63. doi:10.1051/0004-6361:20030197. arXiv:astro-ph/0211480

Schuecker P, Finoguenov A, Miniati F, Böhringer H, Briel UG (2004) Probing turbulence in the Coma galaxy cluster. A\&A 426:387-397. doi:10.1051/0004-6361:20041039. arXiv:astro-ph/0404132

Schwartz SJ, Thomsen MF, Bame SJ, Stansberry J (1988) Electron heating and the potential jump across fast mode shocks. J Geophys Res 93:12,923-12,931

Serlemitsos PJ, Smith BW, Boldt EA, Holt SS, Swank JH (1977) X-radiation from clusters of galaxiesspectral evidence for a hot evolved gas. ApJ 211:L63-L66

Shapiro PR, Moore RT (1976) Time-dependent radiative cooling of a hot, diffuse cosmic gas, and the emergent X-ray spectrum. ApJ 207:460-483. doi:10.1086/154515

Shapiro PR, Moore RT (1977) The nonequilibrium ionization of solar flare coronal plasma and the emergent X-ray spectrum. ApJ 217:621-643. doi:10.1086/155609

Simionescu A, Böhringer H, Brüggen M, Finoguenov A (2007) The gaseous atmosphere of M87 seen with XMM-Newton. A\&A 465:749. arXiv:0709.4499

Simionescu A, Werner N, Finoguenov A, Böhringer H, Brüggen M (2008) Metal-rich multi-phase gas in M 87. AGN-driven metal transport, magnetic-field supported multi-temperature gas, and constraints on non-thermal emission observed with XMM-Newton. A\&A 482:97-112. doi:10.1051/0004-6361: 20078749. arXiv:0709.4499 
Simionescu A, Roediger E, Nulsen PEJ, Brüggen M, Forman WR, Böhringer H, Werner N, Finoguenov A (2009a) The large-scale shock in the cluster of galaxies Hydra A. A\&A 495:721. ArXiv e-prints 0810.0271

Simionescu A, Werner N, Böhringer H, Kaastra JS, Finoguenov A, Brüggen M, Nulsen PEJ (2009b) Chemical enrichment in the cluster of galaxies Hydra A. A\&A 493:409-424. doi:10.1051/0004-6361: 200810225. arXiv:0809.2613

Smith RK, Brickhouse NS, Liedahl DA, Raymond JC (2001) Collisional plasma models with APEC/APED: emission-line diagnostics of hydrogen-like and helium-like Ions. ApJ 556:L91-L95. doi:10.1086/ 322992. arXiv:astro-ph/0106478

Sparks WB, Donahue M, Jordán A, Ferrarese L, Côté P (2004) X-ray and optical filaments in M87. ApJ 607:294-301. doi:10.1086/383189. arXiv:astro-ph/0402204

Spitzer LJ, Greenstein JL (1951) Continuous emission from planetary nebulae. ApJ 114:407. doi:10.1086/ 145480

Springel V, Farrar GR (2007) The speed of the 'bullet' in the merging galaxy cluster 1E0657-56. MNRAS 380:911-925. doi:10.1111/j.1365-2966.2007.12159.x. arXiv:astro-ph/0703232

Sunyaev RA, Norman ML, Bryan GL (2003) On the detectability of turbulence and bulk flows in X-ray clusters. Astron Lett 29:783-790

Sutherland RS, Dopita MA (1993) Cooling functions for low-density astrophysical plasmas. ApJS 88:253327. doi:10.1086/191823

Takahashi T, Kelley R, Mitsuda K, Kunieda H, Petre R, White N, Dotani T, Fujimoto R, Fukazawa Y, Hayashida K, Ishida M, Ishisaki Y, Kokubun M, Makishima K, Koyama K, Madejski GM, Mori K, Mushotzky R, Nakazawa K, Ogasaka Y, Ohashi T, Ozaki M, Tajima H, Tashiro M, Terada Y, Tsunemi H, Tsuru TG, Ueda Y, Yamasaki N, Watanabe S, The ASTRO-H Team (2009) The ASTRO-H Mission. In: Kawai N, Mihara T, Kohama M, Suzuki M (eds) Astrophysics with All-Sky X-ray observations, Proceedings of the RIKEN symposium, held 10-12 June, 2008. RIKEN, and JAXA Suzuki Umetaro Hall, RIKEN Wako, Saitama, Japan, p 356

Tamura T, Bleeker JAM, Kaastra JS, Ferrigno C, Molendi S (2001) XMM-Newton observations of the cluster of galaxies Abell 496. Measurements of the elemental abundances in the intracluster medium. A\&A 379:107-114

Tamura T, Kaastra JS, den Herder JWA, Bleeker JAM, Peterson JR (2004) The elemental abundances in the intracluster medium as observed with XMM-Newton. A\&A 420:135

Tokoi K, Sato K, Ishisaki Y, Ohashi T, Yamasaki NY, Nakazawa K, Matsushita K, Fukazawa Y, Hoshino A, Tamura T, Egawa C, Kawano N, Ota N, Isobe N, Kawaharada M, Awaki H, Hughes JP (2008) Suzaku observation of HCG 62: temperature, abundance, and extended hard X-ray emission profiles. PASJ 60:317. arXiv:0711.1454

Tozzi P, Norman C (2001) The evolution of X-ray clusters and the entropy of the intracluster medium. ApJ 546:63-84. doi:10.1086/318237. arXiv:astro-ph/0003289

Tsujimoto T, Nomoto K, Yoshii Y, Hashimoto M, Yanagida S, Thielemann FK (1995) Relative frequencies of Type Ia and Type II supernovae in the chemical evolution of the Galaxy, LMC and SMC. MNRAS 277:945-958

Tucker WH, Koren M (1971) Radiation from a high-temperature low-density plasma: the X-ray spectrum of the Solar Corona. ApJ 168:283. doi:10.1086/151083

Vikhlinin A (2006) Predicting a single-temperature fit to multicomponent thermal plasma spectra. ApJ 640:710-715. doi:10.1086/500121. arXiv:astro-ph/0504098

Vikhlinin A, Voevodkin A, Mullis CR, VanSpeybroeck L, Quintana H, McNamara BR, Gioia I, Hornstrup A, Henry JP, Forman WR, Jones C (2003) Cosmological constraints from the evolution of the cluster Baryon mass function at $z \sim 0.5$. ApJ 590:15-25. doi:10.1086/374863. arXiv:astro-ph/0212075

Vikhlinin A, Markevitch M, Murray SS, Jones C, Forman W, Van Speybroeck L (2005) Chandra temperature profiles for a sample of nearby relaxed galaxy clusters. ApJ 628:655-672. doi:10.1086/431142. arXiv:astro-ph/0412306

Vikhlinin A, Kravtsov A, Forman W, Jones C, Markevitch M, Murray SS, Van Speybroeck L (2006) Chandra sample of nearby relaxed galaxy clusters: mass, gas fraction, and mass-temperature relation. ApJ 640:691-709. doi:10.1086/500288. arXiv:astro-ph/0507092

Vikhlinin A, Kravtsov AV, Burenin RA, Ebeling H, Forman WR, Hornstrup A, Jones C, Murray SS, Nagai D, Quintana H, Voevodkin A (2009) Chandra cluster cosmology project III: cosmological parameter constraints. ApJ 692:1060-1074. doi:10.1088/0004-637X/692/2/1060. arXiv:0812.2720 
Voigt LM, Fabian AC (2006) Galaxy cluster mass profiles. MNRAS 368:518-533. doi:10.1111/j. 1365-2966.2006.10199.x. arXiv:astro-ph/0602373

Voit GM (2005) Tracing cosmic evolution with clusters of galaxies. Rev Modern Phys 77:207-258. doi:10. 1103/RevModPhys.77.207. arXiv:astro-ph/0410173

Voit GM, Balogh ML, Bower RG, Lacey CG, Bryan GL (2003) On the origin of intracluster entropy. ApJ 593:272-290. doi:10.1086/376499. arXiv:astro-ph/0304447

Werner N, Böhringer H, Kaastra JS, de Plaa J, Simionescu A, Vink J (2006a) XMM-Newton high-resolution spectroscopy reveals the chemical evolution of M 87. A\&A 459:353-360. doi:10.1051/0004-6361: 20065678. arXiv:astro-ph/0608177

Werner N, de Plaa J, Kaastra JS, Vink J, Bleeker JAM, Tamura T, Peterson JR, Verbunt F (2006b) XMM-Newton spectroscopy of the cluster of galaxies 2A 0335+096. A\&A 449:475-491. doi:10.1051/0004-6361: 20053868

Werner N, Finoguenov A, Kaastra JS, Simionescu A, Dietrich JP, Vink J, Böhringer H (2008) Detection of hot gas in the filament connecting the clusters of galaxies Abell 222 and Abell 223. A\&A 482:L29-L33. doi:10.1051/0004-6361:200809599. arXiv:0803.2525

Werner N, Zhuravleva I, Churazov E, Simionescu A, Allen SW, Forman W, Jones C, Kaastra JS (2009) Constraints on turbulent pressure in the X-ray halos of giant elliptical galaxies from resonant scattering. MNRAS. arXiv 0904.0254 (in press)

White DA (2000) Deconvolution of ASCA X-ray data-II. Radial temperature and metallicity profiles for 106 galaxy clusters. MNRAS 312:663-688

White SDM, Briel UG, Henry JP (1993) X-ray archaeology in the Coma cluster. MNRAS 261:L8-L12

Wik DR, Sarazin CL, Finoguenov A, Matsushita K, Nakazawa K, Clarke TE (2009) A Suzaku search for nonthermal emission at hard X-ray energies in the Coma Cluster. ApJ 696:1700-1711. doi:10.1088/ 0004-637X/696/2/1700. arXiv:0902.3658

Woosley SE, Kasen D, Blinnikov S, Sorokina E (2007) Type Ia supernova light curves. ApJ 662:487-503. doi:10.1086/513732. arXiv:astro-ph/0609562

Xu H, Kahn SM, Peterson JR, Behar E, Paerels FBS, Mushotzky RF, Jernigan JG, Brinkman AC, Makishima K (2002) High-resolution observations of the elliptical galaxy NGC 4636 with the reflection grating spectrometer on board XMM-Newton. ApJ 579:600-606. doi:10.1086/342828

Zhang YY, Finoguenov A, Böhringer H, Kneib JP, Smith GP, Czoske O, Soucail G (2007) Scaling relations and mass calibration of the X-ray luminous galaxy clusters at redshift $~ 0.2$ : XMM-Newton observations. A\&A 467:437-457. doi:10.1051/0004-6361:20066567. arXiv:astro-ph/0702739

Zhang Y-Y, Finoguenov A, Böhringer H, Kneib J-P, Smith GP, Kneissl R, Okabe N, Dahle H (2008) LoCuSS: comparison of observed X-ray and lensing galaxy cluster scaling relations with simulations. A\&A 482:451-472

Zwicky F (1937) On the masses of Nebulae and of clusters of Nebulae. ApJ 86:217 